\title{
Outcome of SARS-CoV-2 infection is linked to MAIT cell activation and cytotoxicity
}

\author{
Héloïse Flament ${ }^{1,2,17}$, Matthieu Rouland ${ }^{3}{ }^{3,17}$, Lucie Beaudoin ${ }^{3,17}$, Amine Toubal ${ }^{3,17}$, \\ Léo Bertrand $\mathbb{D}^{3}$, Samuel Lebourgeois ${ }^{4,5}$, Camille Rousseau ${ }^{3}$, Pauline Soulard ${ }^{3}$, \\ Zouriatou Gouda ${ }^{3}$, Lucie Cagninacci ${ }^{3}$, Antoine C. Monteiro ${ }^{4,5}$, Margarita Hurtado-Nedelec ${ }^{1,2}$, \\ Sandrine Luce ${ }^{10}{ }^{3}$, Karine Bailly ${ }^{3}$, Muriel Andrieu ${ }^{3}$, Benjamin Saintpierre ${ }^{3}$, Franck Letourneur ${ }^{3}$, \\ Youenn Jouan ${ }^{6,7}$, Mustapha Si-Tahar ${ }^{6}$, Thomas Baranek ${ }^{6}$, Christophe Paget ${ }^{6}$, Christian Boitard ${ }^{3,8}$, \\ Anaïs Vallet-Pichard ${ }^{3,9}$, Jean-François Gautier ${ }^{10}$, Nadine Ajzenberg ${ }^{11,12}$, Benjamin Terrier ${ }^{13}$, \\ Frédéric Pène ${ }^{3,14}$, Jade Ghosn ${ }^{5,15}$, Xavier Lescure ${ }^{5,15}$, Yazdan Yazdanpanah ${ }^{5,15}$, Benoit Visseaux ${ }^{4,5}$, \\ Diane Descamps ${ }^{4,5}$, Jean-François Timsit ${ }^{5,16}$, Renato C. Monteiro $\mathbb{B}^{1,2}$ and Agnès Lehuen $\mathbb{B}^{3 凶}$
}

Immune system dysfunction is paramount in coronavirus disease 2019 (COVID-19) severity and fatality rate. Mucosal-associated invariant T (MAIT) cells are innate-like T cells involved in mucosal immunity and protection against viral infections. Here, we studied the immune cell landscape, with emphasis on MAIT cells, in cohorts totaling 208 patients with various stages of disease. MAIT cell frequency is strongly reduced in blood. They display a strong activated and cytotoxic phenotype that is more pronounced in lungs. Blood MAIT cell alterations positively correlate with the activation of other innate cells, proinflammatory cytokines, notably interleukin (IL)-18, and with the severity and mortality of severe acute respiratory syndrome coronavirus 2 infection. We also identified a monocyte/macrophage interferon (IFN)- $\alpha-I L-18$ cytokine shift and the ability of infected macrophages to induce the cytotoxicity of MAIT cells in an MR1-dependent manner. Together, our results suggest that altered MAIT cell functions due to IFN- $\alpha-I L-18$ imbalance contribute to disease severity, and their therapeutic manipulation may prevent deleterious inflammation in COVID-19 aggravation.

S evere acute respiratory syndrome coronavirus 2 (SARS-CoV-2) is the etiologic agent responsible for the ongoing outbreak of COVID-19. Cellular targets of SARS-CoV-2 are primarily upper and lower respiratory tract cells, as well as pulmonary cells $s^{1,2}$. The SARS-CoV-2 infection results in a wide range of clinical signs, from asymptomatic to life-threatening acute respiratory distress syndrome, caused by a deleterious antiviral immune response in lungs $\mathrm{s}^{3-5}$. In serious cases, the overbalanced local immune response damages the airways and may lead to noncardiogenic pulmonary edema, hypoxia and the need for artificial ventilation and/or oxygenation $^{6,7}$

Innate-like $\mathrm{T}$ cells, including MAIT, invariant natural killer $\mathrm{T}$ (iNKT) and $\gamma \delta$ T cells, are known to be key actors of pulmonary mucosal immunity, of mucosal tissue repair and are involved in the immune response against numerous respiratory pathogens ${ }^{8-12}$. Innate lymphoid cells (ILCs) have also emerged as important mediators in tissue protection and repair during lung viral infection ${ }^{13}$.
Among them, MAIT cells recognize bacterial metabolites derived from the riboflavin synthesis pathway and presented by the major histocompatibility complex class I-related protein (MR1) ${ }^{14,15}$. It has been established that MAIT cells are activated during viral infections, especially in blood and lungs ${ }^{16-18}$. MAIT cell activation by viruses is T cell antigen receptor (TCR) independent and cytokine dependent ${ }^{16,18}$. During acute and chronic viral infections, MAIT cell blood frequency is reduced while expression of HLA-DR, programmed cell death protein 1, CD38 and CD69 is upregulated ${ }^{16-21}$. Upon acute viral infection, MAIT cells produce high levels of granzyme B $(\mathrm{GzB})^{18}$.

In patients with COVID-19, alteration of peripheral lymphocyte and myeloid subsets is associated with clinical characteristics and treatment efficiency ${ }^{22-24}$. MAIT cell frequency is decreased in the blood of patients with COVID-19 (refs. ${ }^{25,26}$ ); however, their functional contribution to disease remains poorly understood. Here, we analyzed blood and lung MAIT cells of patients with COVID-19

\footnotetext{
'Laboratory of Immunological Dysfunction, Assistance Publique-Hôpitaux de Paris (AP-HP), Bichat-Claude Bernard University Hospital, Paris, France. ${ }^{2}$ Université de Paris, Center for Research on Inflammation, Inserm U1149 \& CNRS ERL8252, Inflamex Laboratory, Paris, France. ${ }^{3}$ Université de Paris, Institut Cochin, Inserm U1016, Centre National de la Recherche Scientifique UMR 8104, Inflamex Laboratory, Paris, France. ${ }^{4}$ Department of Virology, AP-HP, Bichat-Claude Bernard University Hospital, Paris, France. ${ }^{5}$ Université de Paris, Infections Antimicrobials Modelling Evolution UMR 1137, Paris, France. ${ }^{6}$ Université de Tours, Inserm, Centre d'Etude des Pathologies Respiratoires UMR 1100, Tours, France. ${ }^{7}$ Intensive Care Medical Unit, Tours Regional University Hospital, Tours, France. ${ }^{8}$ Department of Diabetology, AP-HP, Cochin University Hospital, Paris, France. ${ }^{9}$ Department of Hepatology, AP-HP, Cochin University Hospital, Paris, France. ${ }^{10}$ Department of Diabetes and Endocrinology, AP-HP, Lariboisière Hospital, Paris, France. "Department of Hematology, AP-HP, Bichat-Claude Bernard University Hospital, Paris, France. ${ }^{12}$ Université de Paris, LVTS, Inserm, Paris, France. ${ }^{13}$ Department of Internal Medicine, AP-HP, Cochin University Hospital, Paris, France. ${ }^{14}$ Medical Intensive Care Unit, AP-HP, Cochin University Hospital, Paris, France. ${ }^{15}$ Department of Infectious and Tropical Diseases, AP-HP, Bichat-Claude Bernard University Hospital, Paris, France. ${ }^{16}$ Medical and Infectious Diseases Intensive Care Unit, AP-HP, Bichat-Claude Bernard University Hospital, Paris, France. ${ }^{17}$ These authors contributed equally: Héloïse Flament, Matthieu Rouland, Lucie Beaudoin, Amine Toubal. e-mail: agnes.lehuen@inserm.fr
} 
$\mathbf{a}$

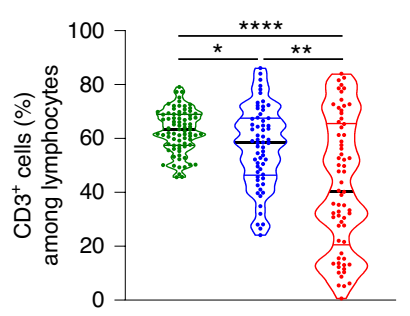

C

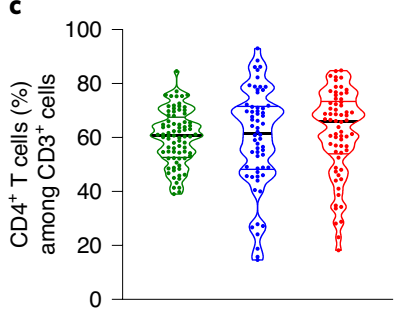

b
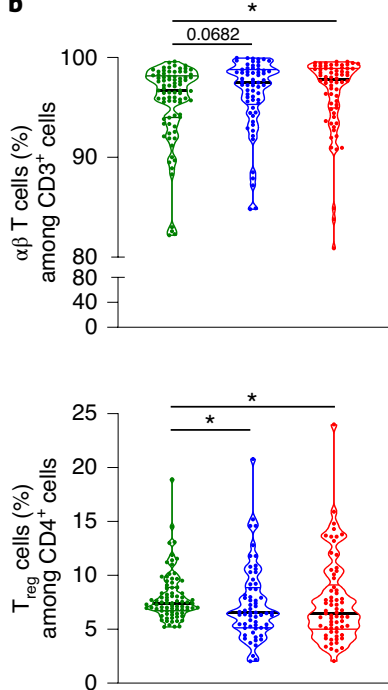
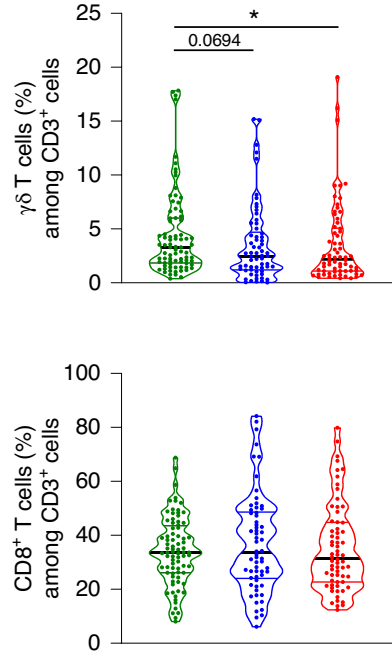

d
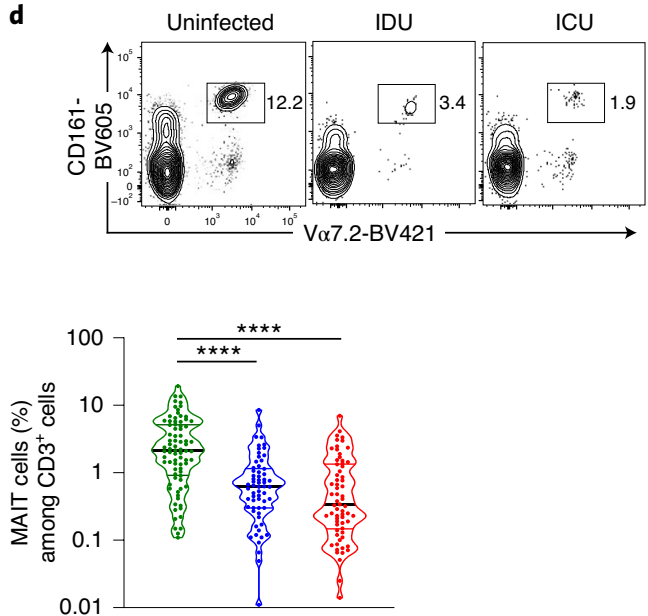
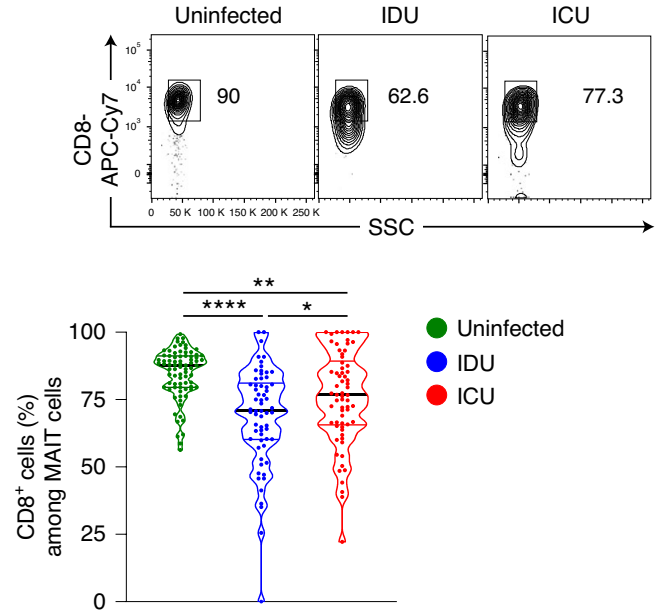

Fig. 1 | Immune cell frequencies and status in the blood of patients with COVID-19. a,b, Whole blood or PBMCs were collected from patients and analyzed through flow cytometry. Flow cytometry analysis of $C D 3^{+}$cell $(\mathbf{a}), \alpha \beta T$ cell and $\gamma \delta \mathrm{T}$ cell frequencies (b) from uninfected controls ( $n=80$ ),

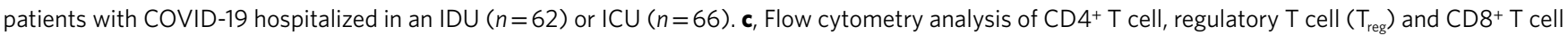
frequencies in the blood of patients as described in $\mathbf{a}$ and $\mathbf{b}$. d,e, Representative quantile contour plots of $\mathrm{V} \alpha 7.2$ and CD161 staining to identify MAIT cells in the blood of uninfected controls and patients with COVID-19 from IDU and ICU, and MAIT cell (d) and CD8 ${ }^{+}$MAIT cell (e) frequencies in the blood of patients as described in $\mathbf{a}$ and $\mathbf{b}$. SSC, side scatter. Small horizontal lines indicate the median and quartiles. Each symbol represents one biological sample. ${ }^{\star} P<0.05,{ }^{\star \star} P<0.01$ and ${ }^{\star \star \star \star} P<0.0001$ (two-sided Mann-Whitney non-parametric test).

who had differences in disease severity status. These patients were compared with uninfected controls matched for age, sex and comorbidities. Mechanisms of action were studied using in vitro experiments with cultured macrophages from healthy donors, infected with SARS-CoV-2 and cocultured with blood MAIT cells from the same donors. Our study provides new insights on the cytotoxic function of MAIT cells in SARS-CoV-2 infection and the critical role of the IFN- $\alpha$-IL-18 shift in monocytes and macrophages.

\section{Results}

Adaptive and innate $\mathrm{T}$ cell frequency in the blood of patients with COVID-19. We first began our study of immune cells in SARS-CoV-2 infection by analyzing the frequency and phenotype of lymphocytes in blood samples from patients with COVID-19, as well as (age-matched and body mass index (BMI)-matched) uninfected donors (Extended Data Fig. 1). Fifty-one patients with COVID-19 had been admitted to an infectious disease unit (IDU; moderate cases) and 51 patients to an intensive care unit (ICU; severe cases, with a $41 \%$ death rate). As controls, we included 80 healthy uninfected donors, including donors with various pathologies (for example, diabetes and obesity) to match those affecting hospitalized patients with COVID-19 (Extended Data Fig. 1). Characteristics and health data of recruited patients and controls are listed in Supplementary Table 1. Whole blood was collected and stained for flow cytometry to analyze frequencies of innate and adaptive T lymphocytes following the gating strategies presented in Supplementary Fig. 1. A majority of patients with COVID-19 presented a significant reduction of T cells in the IDU, more pronounced in ICU patients (Fig. 1a), confirming earlier reports ${ }^{27,28}$. Among $\mathrm{CD}^{+} \mathrm{T}$ cells, there was a slight increase in blood $\alpha \beta \mathrm{T}$ cell frequency in ICU patients, mirrored by a decrease of $\gamma \delta \mathrm{T}$ cells (Fig. 1b). $\mathrm{CD}^{+}$and $\mathrm{CD}^{+} \mathrm{T}$ cell frequencies among $\mathrm{CD}^{+} \mathrm{T}$ cells were not significantly impacted by SARS-CoV-2 infection, whereas regulatory $\mathrm{T}$ cell frequency was significantly reduced in IDU and ICU 
patients compared to uninfected controls (Fig. 1c). We observed a collapse of $\mathrm{V} \alpha 7.2^{+} \mathrm{CD} 161^{+}$MAIT cell frequency among $\mathrm{CD}^{+}$cells in patients with COVID-19, down to a tenth of those observed in uninfected controls (Fig. 1d). We confirmed that MAIT cell markers CD161 and V $\alpha 7.2$ allowed MAIT cell identification similar to that found with MR1 tetramers loaded with the active ligand 5-(2-oxopropylideneamino)-6-D-ribitylaminouracil (5-OP-RU) in all three groups of patients (Extended Data Fig. 2a-c). Among MAIT cells, the $\mathrm{CD}^{+}$subset was further reduced in patients with COVID-19, but to a lesser extent in ICU compared to IDU patients (Fig. 1e). We therefore observed a decrease of blood MAIT cells in patients with COVID-19, with an alteration of MAIT cell subset repartition.

MAIT cells are activated and cytotoxic in patients with COVID19. As MAIT cell frequency is reduced in the blood of patients with COVID-19, we analyzed their surface marker expression and their cytokine production. An activated phenotype with an increase of CD69 expression was observed in patients with COVID-19, with a median of $41 \%$ CD $69^{+}$MAIT cells in IDU patients and $67 \%$ in ICU patients, with some reaching $100 \%$ (Fig. 2a). Blood MAIT cells also displayed a significant increase of NK cell-associated activation $\mathrm{CD} 6^{+}$marker compared to controls, which was highest in ICU patients (Fig. 2a). Accordingly, double-positive $\mathrm{CD}^{2} 6^{+} \mathrm{CD} 69^{+}$ MAIT cell frequency was increased in IDU patients and even more in ICU patients (Fig. 2a). The frequency of MAIT cells coexpressing CCR6, the receptor for the CCL20 chemokine (a tissue-migrating marker) and survival CD127 marker was decreased, suggesting that low circulating MAIT cell frequency might reflect migration into inflamed infected tissues and/or activation-induced cell death (Extended Data Fig. 3a). MAIT cell activation in some patients might be associated with secondary infections and presence of bacteria in the blood (Extended Data Fig. 3b).

In contrast to MAIT cells, $\mathrm{CD}^{+} 9^{+}$expression on $\mathrm{CD}^{+} \mathrm{T}$ cells remained moderate (median of 10\%) in IDU and ICU patients although increased compared to uninfected controls (Extended Data Fig. 3c). The frequency of $\mathrm{CD} 56^{+} \mathrm{CD} 8^{+} \mathrm{T}$ cells was similar in all three groups of patients. Consequently, the frequency of effector $\mathrm{CD}^{2} 6^{+} \mathrm{CD} 69^{+} \mathrm{CD} 8^{+} \mathrm{T}$ cells was modestly increased in patients with COVID-19 compared to uninfected controls (Extended Data Fig. 3c).

We next investigated the phenotype of MAIT cells, with unsupervised methods, on nine flow cytometry parameters. We first compared MAIT cells of patients with COVID-19 to those of uninfected controls by multidimensional scaling (MDS) plots, which revealed a progression of severity from uninfected controls to IDU and ICU patients (Fig. 2b). We next performed graphical dimensional reduction by $t$-distributed stochastic neighbor embedding ( $t$-SNE) and uniform manifold approximation and projection (UMAP), which also returned a progressive distribution of MAIT cells from controls to IDU and ICU patients (Fig. 2c and Extended Data Fig. 4a,b). Both CD69 and CD56 expression distributions were the most closely matched with the distribution of MAIT cells according to COVID-19 severity (highest expression at highest severity; Fig. 2d and Extended Data Fig. 4c).

MAIT cell function was then assessed after phorbol myristate acetate (PMA)-ionomycin stimulation by analyzing IFN- $\gamma$ and GzB production in all three groups of patients and IL-2, IL-4, IL-10, IL-17 and TNF in IDU and ICU patients (Fig. 2e,f and Extended Data Fig. 5a). Production of IFN- $\gamma$ was decreased in COVID-19 IDU patients compared to uninfected controls. However, IFN- $\gamma$ and IL-2 increased in ICU patients compared to IDU patients (Fig. 2e and Extended Data Fig. 5a). In contrast, GzB production progressively increased in stimulated MAIT cells in IDU and ICU patients as compared to controls. Elevated GzB production was also detected in unstimulated MAIT cells from infected patients (Fig. 2f,g and Extended Data Fig. 5a,b). Such enhanced cytokine and GzB production in ICU patients was not observed in conventional $\alpha \beta \mathrm{T}$, $\gamma \delta \mathrm{T}$ and CD3- cells (including NK cells; Extended Data Fig. 5a,b). While we observed a slight increase in IL-17 and IFN- $\gamma$ production by MAIT cells in ICU patients when compared to IDU patients, we did not observe any increase of IL-17+IFN- $\gamma^{+}$double-positive MAIT cells in patients with COVID-19 (Extended Data Fig. 5c). Finally, we analyzed GzB production by lung MAIT cells from endotracheal aspirates (ETAs) and bronchoalveolar lavages (BALs) of ICU patients. MAIT cells from both fluids produced more GzB than their counterparts from blood in the same patients (Fig. $2 \mathrm{~h}$, Extended Data Figs. 2c and 5d). Altogether, these results show that blood MAIT cells from patients with COVID-19 display an activated/effector phenotype and cytotoxic function associated with disease severity. This cytotoxic phenotype is even more pronounced in lungs of patients infected with SARS-CoV-2.

Links between MAIT cell activation and other innate immune cell alterations. Following MAIT cell analyses, we further investigated other innate-like immune cell frequencies, phenotypes and activation in the blood of patients infected with SARS-CoV-2. A significant reduction in the frequency of NK cells, ILC2 and ILC3 in IDU and ICU patients was observed, which was more pronounced for NK cells and ILC2 in patients with COVID-19 from an ICU (Fig. 3a). CD69 expression was higher in all infected patients on NK cells, ILC3 and $\gamma \delta$ T cells, whereas it was reduced on ILC2 (Fig. 3b).

We sought to identify correlations between MAIT cells and other innate cells in patients with COVID-19. A multiparametric matrix correlation plot showed strong positive correlations between frequencies of activated MAIT cells (CD56 ${ }^{+}$and/or CD69 ${ }^{+}$ MAIT cells) with CD69+ ILC3, NK cell and $\gamma \delta$ T cell frequencies, as well as $\mathrm{CD} 69^{+} \mathrm{CD} 56^{+} \gamma \delta$ T cells (Fig. $\left.3 c, d\right)$. Several negative correlations were also observed between activation of these populations and their frequencies. CD69 expression on MAIT cells was negatively correlated with ILC3, ILC2 and NK cell frequencies (Fig. 3c). Cytokine production by MAIT cells was also negatively correlated with ILC3 frequencies and to a lesser extent with ILC2 and NK cell frequencies. Taken together, these data suggest that inflammatory

Fig. 2 | Blood MAIT cells are activated and secrete proinflammatory cytokines in patients with COVID-19. a, Representative quantile contour plots of CD69, CD56 and double-positive surface marker expression on blood MAIT cells from one uninfected control and two patients with COVID-19 who were admitted to an IDU and an ICU, respectively. CD69+, CD56 and CD56+CD69+ MAIT cell frequencies in the blood of uninfected controls ( $n=80$ ) and infected patients from the IDU $(n=62)$ and ICU $(n=66)$. b,c, MDS plot (b) and UMAP $(\mathbf{c})$. c, Analysis of MAIT cells for each uninfected $(n=23)$ and infected patient from the IDU $(n=50)$ or ICU $(n=66)$ from Bichat hospital analyzed with the same flow cytometer. d, UMAP divided by groups and colored by the scaled expression of CD69 and CD56. e,f, Representative quantile contour plots and frequencies of IFN- $\gamma(\mathbf{e})$ and GzB (f) in MAIT cells after stimulation from uninfected controls $(n=25-27)$ and patients with COVID-19 hospitalized in an IDU $(n=14-15)$ or ICU $(n=15)$. $\mathbf{g}$, Representative quantile contour plots and frequencies of $\mathrm{GzB}^{+}$MAIT cells without stimulation in uninfected controls $(n=19)$ and patients in an IDU $(n=11)$ or ICU $(n=14)$ with COVID-19. $\mathbf{h}$, Comparative analysis of frequencies of GzB+ MAIT cells without stimulation in blood and ETAs of patients in an ICU with COVID-19 ( $n=7$ ). Representative quantile contour plots are shown on the left, and individuals are shown on the right. Small horizontal lines indicate the median and quartiles (a) or the median (e-g). Each symbol represents one biological sample. ${ }^{\star} P<0.05,{ }^{\star \star} P<0.01,{ }^{\star \star \star} P<0.001$ and ${ }^{\star \star \star \star} P<0.0001$ (two-sided Mann-Whitney non-parametric test (a and $\mathbf{e}-\mathbf{g}$ ) and Wilcoxon signed-rank test $(\mathbf{h})$ ). 
processes in patients infected with SARS-CoV-2 involve concomitant activation of MAIT cells with other innate immune cells associated with loss of these population frequencies in blood.
Fatal SARS-CoV-2 infection is linked with activation and function of MAIT cells. To investigate the impact of immune cell populations on disease outcome, blood samples were analyzed by a
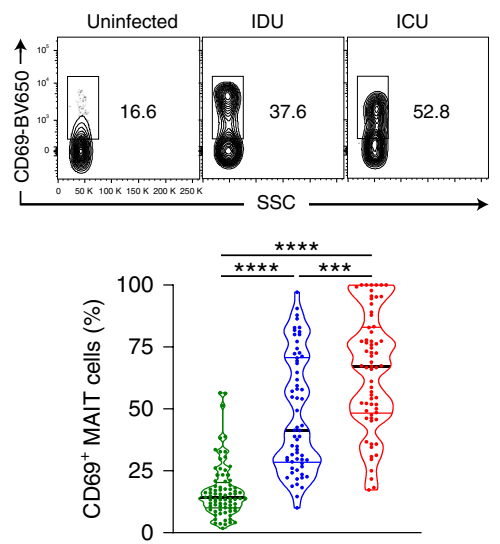

b

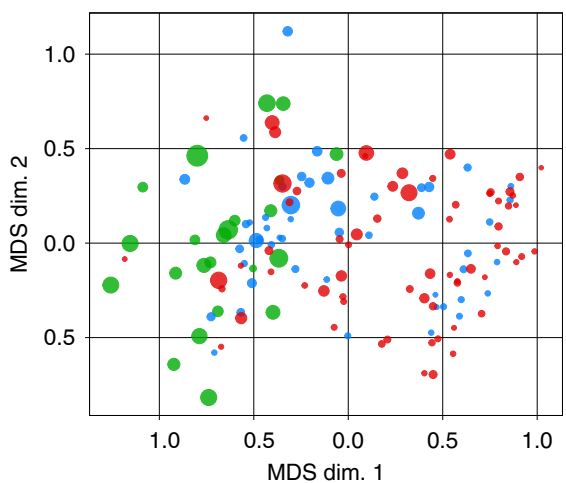

e
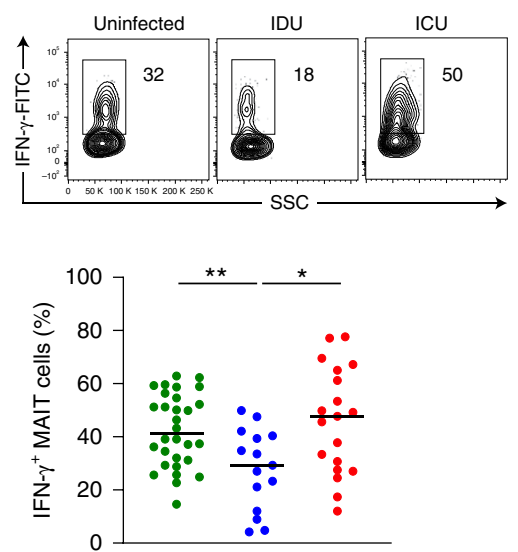

h

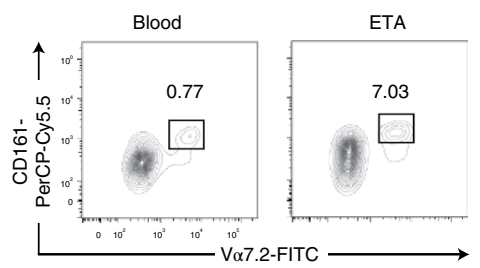

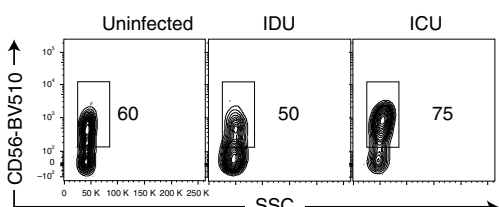

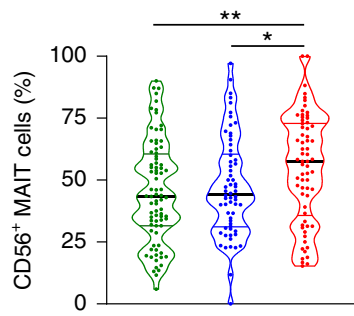

c

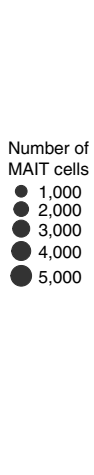

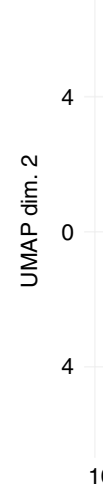
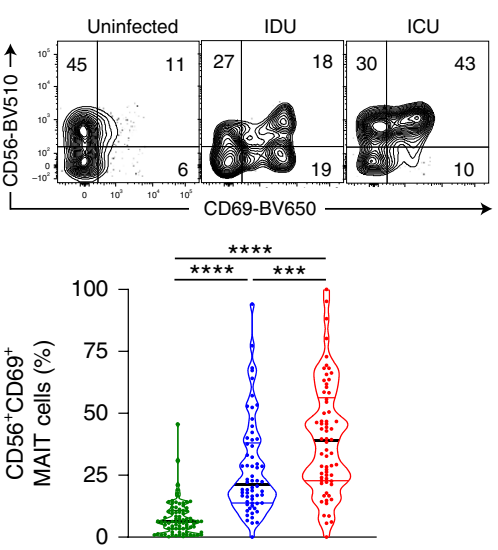

d Uninfected IDU ICU CD69
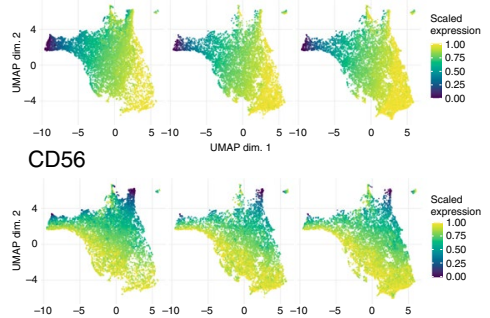

Uninfected

IDU

$\begin{array}{lllll}10 & 5 & 0 & 5 & \bigcirc \mathrm{ICU}\end{array}$

UMAP dim. 1
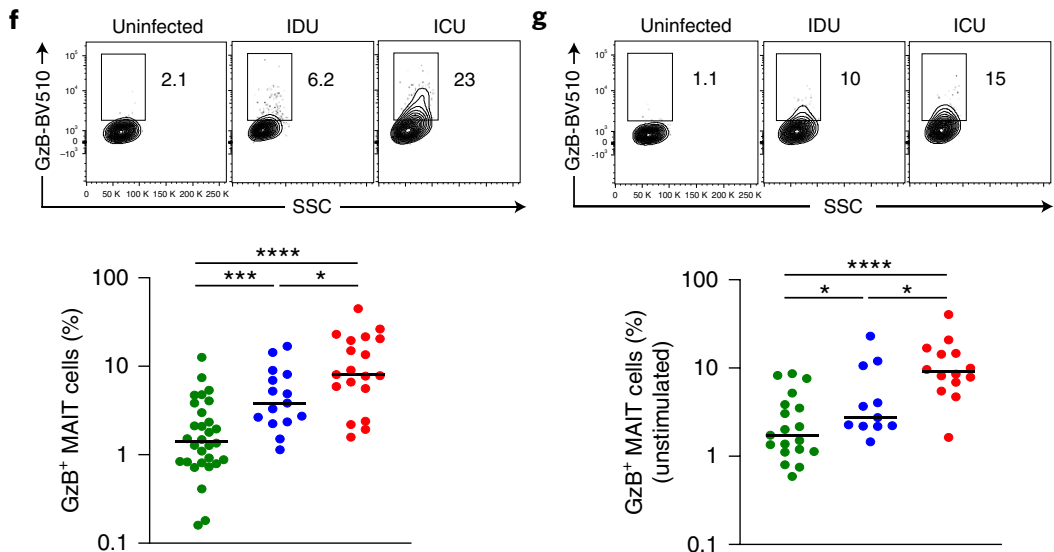
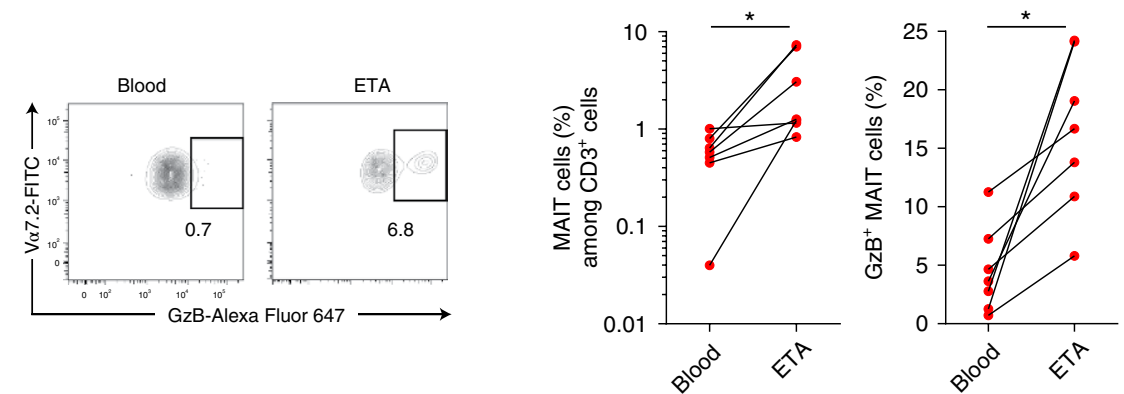


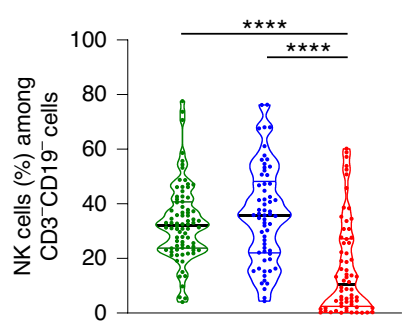

b

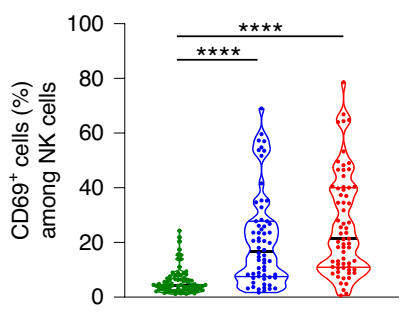

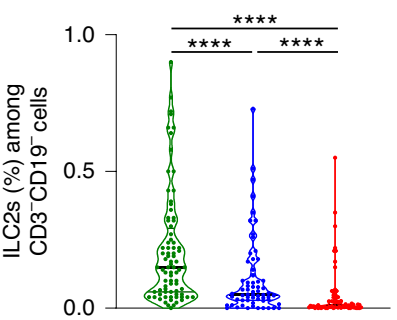

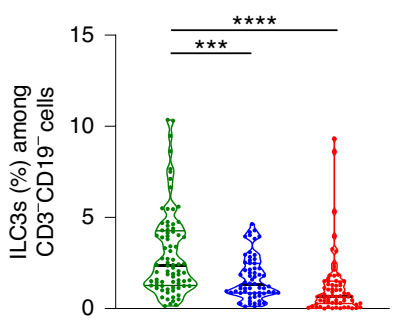

Uninfected

ICU
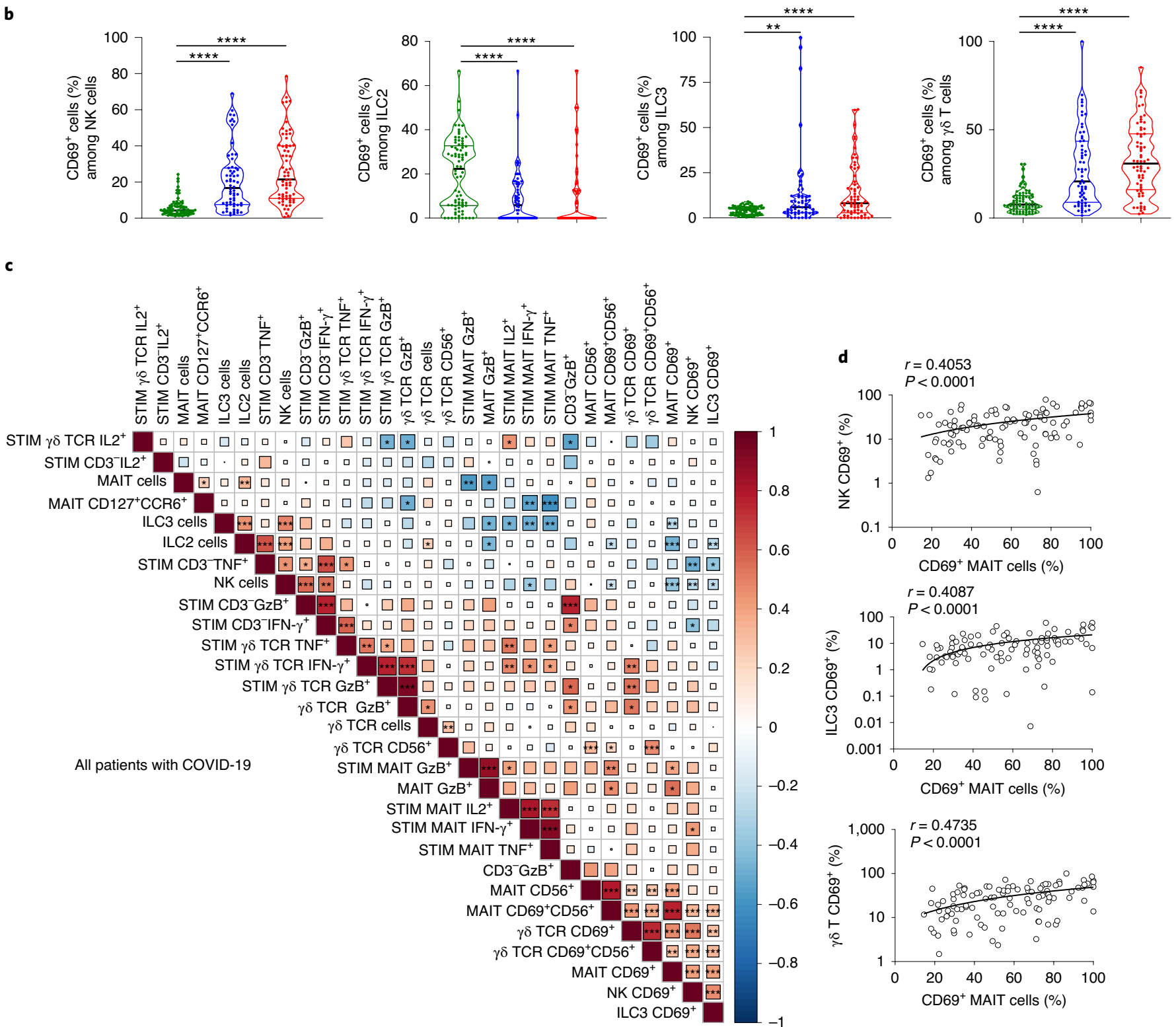

Fig. 3 | MAIT cells phenotype correlates with ILC and $\boldsymbol{\gamma} \boldsymbol{\delta}$ T cell alteration in patients with COVID-19. $\mathbf{a}, \mathbf{b}$, Frequencies of NK, ILC2 and ILC3 innate immune cells among CD3-CD19- cells (a) and CD69 activation surface markers on NK, ILC2, ILC3 and $\gamma \delta$ T cells (b) in uninfected controls ( $n=80$ ) and patients with COVID-19 hospitalized in an IDU $(n=62)$ or ICU $(n=66)$. c. Multiparametric matrix correlation plot of MAIT cell, $\gamma \delta$ T cell, ILC and NK cell frequencies, and surface marker and intracytoplasmic staining in patients with COVID-19. Spearman's correlation coefficients are visualized by square size and color intensity. Variables are ordered by hierarchical clustering. $\mathbf{d}$, Correlation between CD69+ MAIT cells and CD69+ NK, CD69+ ILC3 or CD69+ $\gamma \delta$ T cells in patients with COVID-19 $(n=102)$. Small horizontal lines indicate the median and quartiles ( $\mathbf{a}$ and $\mathbf{b}$ ). Each symbol represents one biological sample. ${ }^{\star} P<0.05,{ }^{\star \star} P<0.01$, ${ }^{\star \star \star} P<0.001$ and ${ }^{\star \star \star \star} P<0.0001$ (two-sided Mann-Whitney non-parametric test ( $\mathbf{a}$ and $\mathbf{b}$ ) and Spearman's non-parametric correlation test corrected for multiple inferences using Holm's method (c and d)).

comparing two groups: surviving versus fatal outcomes of patients with COVID-19. CD69 expression significantly increased on MAIT, CD8 T, $\gamma \delta \mathrm{T}$ and NK cells in fatal patients with COVID-19 compared to surviving IDU or ICU patients (Fig. 4a). MAIT cells displayed the highest activation level in all patients, particularly those with fatal outcomes. We next examined immune cell function relative to 
a

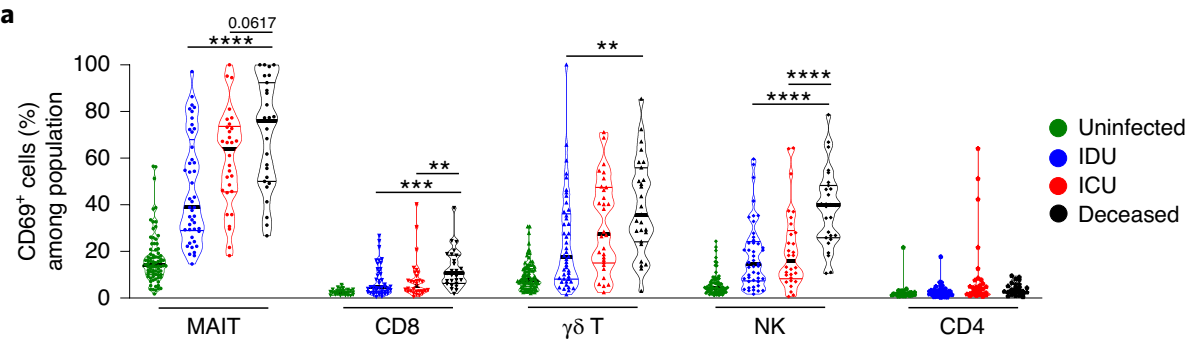

b
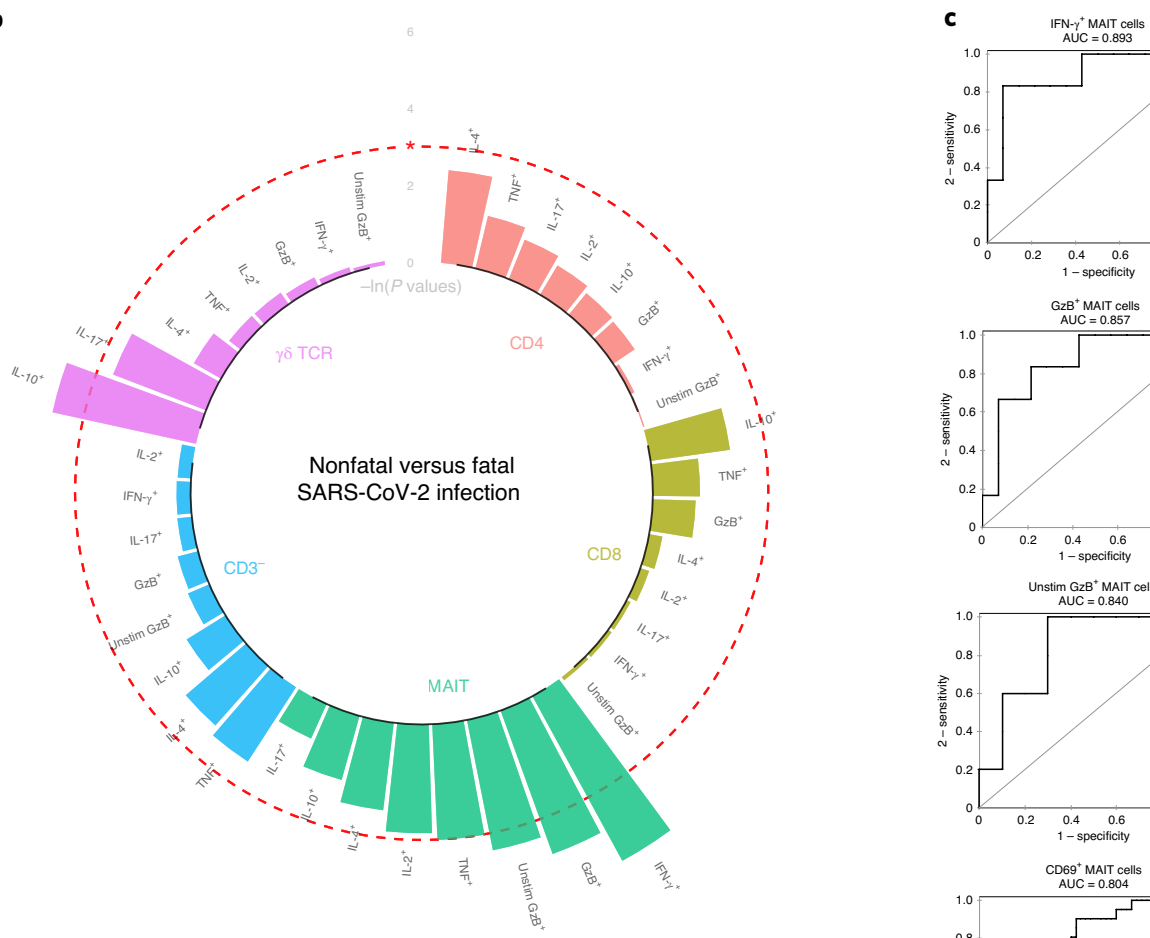

IFN- ${ }^{+}$MAIT cells
AUC $=0.893$
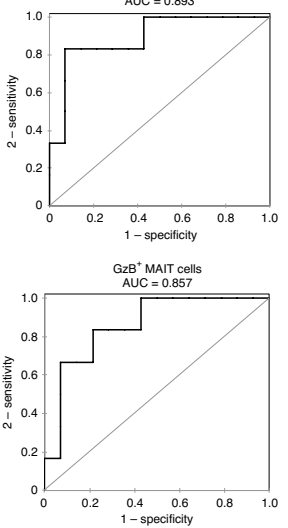

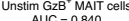

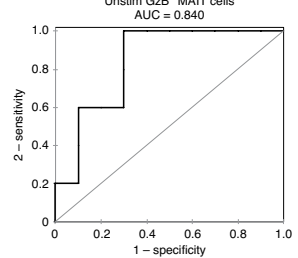

$\mathrm{CDEG}^{+}$MAIT cells
AUC $=0.004$

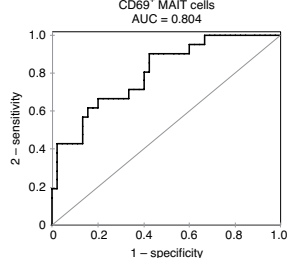

d

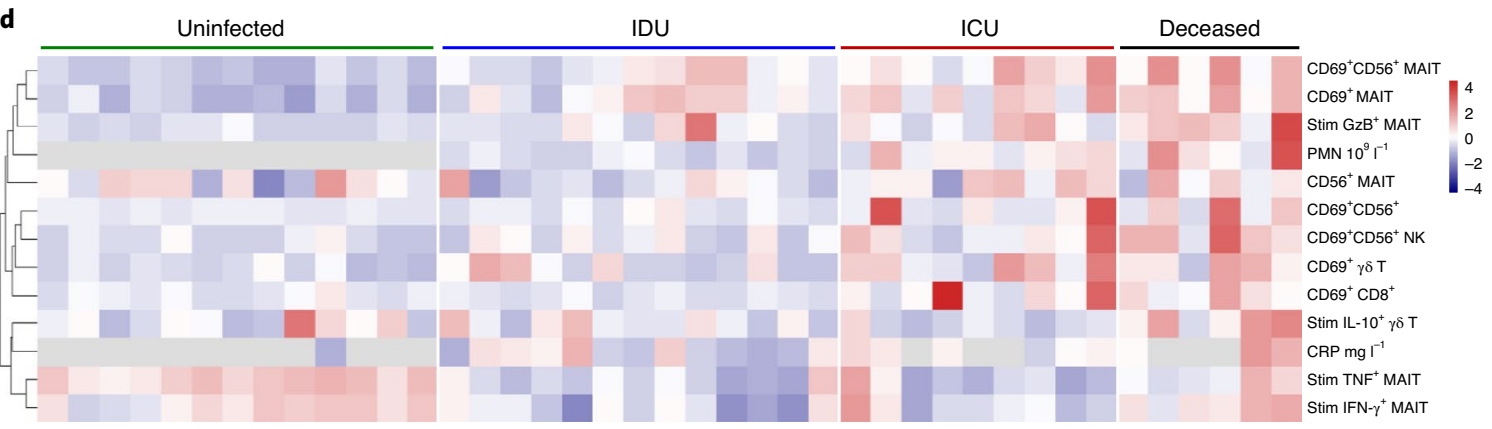

Fig. 4 | MAIT cell activation and functions are altered in fatal as compared to nonfatal COVID-19. a, Frequency of CD69+ cells among MAIT cells, conventional CD8 ${ }^{+}$and $C D 4^{+}$T cells, $\gamma \delta$ T cells and NK cells in the blood of uninfected controls $(n=80)$, surviving patients with COVID-19 hospitalized in an IDU $(n=45)$ or ICU $(n=30)$ and deceased patients from both the IDU and ICU $(n=27)$. b, Circular bar plot represents negative natural logarithm $P$ values of the frequency of immune cell (MAIT, CD8 ${ }^{+}, \mathrm{CD}^{+}, \mathrm{CD} 3^{-}$and $\gamma \delta \mathrm{T}$ ) function, including $\mathrm{IL}-2^{+}, \mathrm{IL}-4^{+}, \mathrm{IL}-10^{+}, \mathrm{IL}-17^{+}, \mathrm{IFN}-\gamma^{+}, \mathrm{TNF}^{+}$and $\mathrm{GzB} \mathrm{B}^{+}$after stimulation or $\mathrm{GzB}^{+}$without stimulation (unstim), between deceased (stimulated, $n=6$; unstimulated, $n=5$ ) and surviving (stimulated, $n=23$, except for MAIT parameters, where $n=22$; unstimulated, $n=16$ ) patients infected with SARS-CoV-2. The red dashed circle represents a $P$ value of 0.05 . $\mathbf{c}$, ROC curves of the predictive MAIT cell markers defining the outcome of COVID-19. AUC, area under the curve. d, Heat map showing the scaled expression of different innate-like immune cell activation (MAIT, ILC3, NK, $\gamma \delta$ T and CD8), CRP and polymorphonuclear neutrophil (PMN) ordered by hierarchical clustering in uninfected controls $(n=13)$ and patients in an IDU $(n=13)$, and nonfatal ICU $(n=9)$ and fatal ICU $(n=6)$ patients. Small horizontal lines indicate the median and quartiles (a). Each symbol (a) or column (d) represents one patient. Upregulated parameters are shown in red, and downregulated parameters are shown in navy blue. ${ }^{\star} P<0.05$, ${ }^{\star \star} P<0.01,{ }^{\star \star \star} P<0.001$ and ${ }^{\star \star \star \star} P<0.0001$ (two-sided Mann-Whitney non-parametric test). 
disease outcome by measuring intracellular cytokines and GzB in stimulated and unstimulated immune cells (Fig. 4b, Extended Data Fig. 6a,b and data not shown). IFN- $\gamma$, tumor necrosis factor (TNF) and GzB in stimulated MAIT cells and GzB in unstimulated MAIT cells were significantly enhanced in deceased patients compared to surviving patients (Fig. $4 \mathrm{~b}$ and Extended Data Fig. 6a,b). IL-10 production by stimulated $\gamma \delta$ T cells in deceased patients was greater compared to surviving patients (Fig. $4 \mathrm{~b}$ and Extended Data Fig. 6a). Thus, MAIT cell function was more associated with mortality than functions of other $\mathrm{T}$ cell populations. No modification of IL-2, IL- 4 and IL-17 production in $\mathrm{CD}^{+}, \mathrm{CD}^{+}, \mathrm{CD}^{-}, \gamma \delta \mathrm{T}$ and MAIT cells was observed in fatal cases (Fig. $4 \mathrm{~b}$ and Extended Data Fig. 6a). Moreover, statistical regression analyses identified four MAIT cell markers (IFN- $\gamma$ production, stimulated GzB production, unstimulated GzB production and CD69) that defined predictive models for COVID-19 outcome, as tested on a receiver operating characteristic (ROC) curve (Fig. 4c). To allow a more global view of individual patients, we generated a heat map based on multiple immune cell and blood parameters from single uninfected, infected and fatal patients with COVID-19. This analysis highlights different activation signatures of nonsurviving patients compared to surviving patients with COVID-19 and controls, confirming that high MAIT and innate cell activation is a signature of fatal outcomes (Fig. 4d). Therefore, high MAIT cell activation and effector activity is associated with and potentially predictive of fatal outcomes in COVID-19.

Increased proinflammatory cytokine levels correlate with blood MAIT cell alterations. We next analyzed plasma levels of several cytokines by cytometric bead array (CBA) and electrochemiluminescence immunoassay in surviving IDU and ICU patients, as well as in nonsurviving patients (Fig. 5a,b). IL-6, IL-8, IL-10, IL-15 and IL-18 levels were significantly increased in the plasma of nonsurviving patients compared to surviving patients, confirming a state of widespread, pronounced inflammation in severe cases of COVID-19 (ref. ${ }^{27}$; Fig. 5a,b). IFN- $\alpha 2$ levels were significantly decreased in ICU compared to IDU patients, although no significant difference between surviving ICU and deceased patients was detected (Fig. 5b). IL-1 $\beta$ levels were similar between all three groups (Fig. 5b). Multiparametric matrix correlation plots showed strong positive correlation of IL-6, IL-8, IL-10, IL-15 and IL-18 levels with frequencies of $\mathrm{CD}^{+} 9^{+}$and $\mathrm{CD} 69^{+} \mathrm{CD} 56^{+}$MAIT cells in the blood of all patients (Extended Data Fig. 7a,b). IFN- $\alpha 2$ blood levels correlated positively with the production of IFN- $\gamma$, TNF and IL- 2 by MAIT cells (Extended Data Fig. 7a).

Separated matrix correlation plot between surviving and nonsurviving ICU patients showed different relationships between blood cytokine concentration and GzB production by MAIT cells. In surviving patients, there was a strong negative correlation between blood IL-15 concentration and spontaneous GzB production by MAIT cells, whereas in nonsurviving patients, there was a strong positive correlation between blood IL-18 concentration and GzB MAIT production. The link between IL-18 and MAIT cell activation is further supported by high IL-18R $\alpha$ expression on all blood MAIT cells in controls and patients infected with SARS-CoV-2 compared to other immune populations (Extended Data Fig. 8a-c). In nonsurviving ICU patients, blood IL-18 concentration was negatively correlated with IFN- $\alpha 2$ blood concentration (Fig. $5 \mathrm{c}$ ). Accordingly, IL-18 blood concentration was increased in long-term ICU patients with COVID-19, whereas IFN- $\alpha 2$ blood concentration was decreased in these patients (Fig. 5d). Further, CD69+ MAIT cell frequency increased with time and was highest one month after symptom onset, when death rate was highest among ICU patients. Our data therefore reveal that proinflammatory cytokines and IL-10 concentration are associated with MAIT cell activation and highlight a unique relationship between plasma IL-18 levels and circulating cytotoxic MAIT cells in fatal cases.

MAIT cell phenotype and functions are associated with SARS-CoV-2 severity and clinical parameters. As previously reported in our cohort, COVID-19 severity was associated with extended pulmonary damage, as evaluated using a chest computed tomography severity score from 1 (mild) to 5 (critical; Fig. 6a). The ratio of partial pressure of arterial oxygen $\left(\mathrm{PaO}_{2}\right)$ to fraction of inspired oxygen $\left(\mathrm{FiO}_{2}\right)$ was significantly reduced and $\mathrm{C}$-reactive protein (CRP) concentrations were significantly increased in ICU patients. Both parameters were more affected in fatal cases (Fig. 6b,c).

A correlation matrix of MAIT cell activation, function and clinical parameters was established for all patients with COVID-19 (Fig. 6d). It included age, BMI, CRP, simplified acute physiology score II (SAPS II; an estimator of patient mortality risk at ICU admission), $\mathrm{PaO}_{2} / \mathrm{FiO}_{2}$ ratio, polymorphonuclear neutrophil (PMN) count, disease duration and pulmonary lesions. Analysis of these clinical indicators considering MAIT cells revealed that $\mathrm{PaO}_{2} / \mathrm{FiO}_{2}$ ratio strongly negatively correlated with $\mathrm{CD} 9^{+}$expression, and IFN- $\gamma$ and TNF production by MAIT cells, whereas SAPS II score positively correlated with CD69+ ${ }^{+}$MAIT cell frequency (Fig. 6d,e). CRP level correlated with MAIT cell activation phenotype and cytokine secretion (IL-2, IFN- $\gamma$ and TNF), while PMN frequency positively correlated with $\mathrm{CD}^{6} 9^{+}$MAIT cell frequency (Fig. 6d,e).

Principal-component analysis (PCA) of all patients with COVID19 showed an unsupervised overview of all clinical data, cytokine concentrations, and immune cell frequencies, activation and functions for each patient with COVID-19 (Fig. 6f). The PCA showed segregation of deceased patients compared to surviving patients, with $\mathrm{CD}^{+} 9^{+}$MAIT cells being the most important contributing variable (Fig. $6 \mathrm{~g}$ and Supplementary Fig. 2). Of note, several parameters also contributed to the surviving versus fatal case discrimination vector such as blood levels of IL-8, IL-15, IL-18, CRP, PMN, activated ILC3 and NK cells, previously found to be correlated to MAIT cell activation (Fig. 3c,d and Fig. 6d,e). Therefore, altered

Fig. 5 | Proinflammatory cytokines are elevated in patients with COVID-19 and correlate with MAIT cell activation and cytokine secretion. a, CBA cytokine quantification of IL-6, IL-8 and IL-10 in the blood of surviving patients with COVID-19 hospitalized in an IDU (IL-6, $n=22 ;$ IL-8, $n=19 ;$ IL-10, $n=19$ ) or ICU (IL-6, $n=28 ;$ IL-8, $n=28 ;$ IL-10, $n=27)$ and of patients with fatal COVID-19 $(n=25)$. b, Cytokine quantification by electrochemiluminescence immunoassay of IFN- $\alpha 2$, IL-15, IL-18 and IL-1 $\beta$ in the blood of surviving patients with COVID-19 hospitalized in an IDU (IFN- $\alpha 2, n=18 ;$ IL-15, $n=18$; IL-18, $n=18 ; \mathrm{IL}-1 \beta, n=14)$ or ICU (IFN- $\alpha 2, n=24 ; \mathrm{IL}-15, n=27 ; \mathrm{IL}-18, n=27 ; \mathrm{IL}-1 \beta, n=16)$ and of deceased patients (IFN- $\alpha 2, n=21 ; \mathrm{IL}-15, n=24 ; \mathrm{IL}-18, n=24$; $\mathrm{IL}-1 \beta, n=20)$. Values under the limit of detection were not statistically computed and are not displayed. $\mathbf{c}$, Multiparametric matrix correlation plot of IL-6, IL-8, IL-15, IL-18 and IFN- $\alpha 2$ cytokine levels in blood; frequencies of CD69+, CD56+, CD69+CD56+ and GzB+ MAIT cells; frequencies of IFN- $\gamma^{+}$, IL-2+ $\mathrm{TNF}^{+}$and GzB+ stimulated MAIT cells; in patients with nonfatal COVID-19 (upper right; $n=30$ ) versus patients with fatal COVID-19 in ICU (lower left; $n=21)$. Spearman's correlation coefficients are visualized by square size and color intensity. Variables are ordered alphabetically. d, CD69+ MAIT cells (IDU, $n=51$; ICU, $n=50$ ), IL-18 (IDU, $n=22$; ICU, $n=47$ ) and IFN- $\alpha 2$ (IDU, $n=22 ;$ ICU, $n=41$ ) blood levels according to symptom duration (days) in patients with COVID-19, starting at the first clinical signs. Small horizontal lines (a and $\mathbf{b}$ ) or columns (d) indicate the median. Each symbol represents one patient. ${ }^{\star} P<0.05,{ }^{\star \star} P<0.01,{ }^{\star \star \star} P<0.001$ and ${ }^{\star \star \star \star} P<0.001$ (two-sided Mann-Whitney non-parametric test (a,b and $\mathbf{d}$ ) and Spearman's non-parametric correlation test corrected for multiple inferences using Holm's method (c)). 
activation markers and cytokine production by blood MAIT cells correlate with clinical parameters and are associated with severity and disease outcome.

MAIT cell alterations in patients with COVID-19 are associated with a monocyte phenotype switch. Several reports have shown that the myeloid compartment was dysregulated in patients with COVID-19 in association with an inflammatory storm ${ }^{23,24,29}$. MAIT cells exert antiviral properties that are promoted especially by macrophages ${ }^{16,18}$. Using a small, second cohort of patients infected with SARS-CoV-2 ( $n=9$ in IDU and $n=13$ in ICU), we studied the link between blood MAIT cells and monocytes (Extended Data Fig. 9). As previously observed in our first cohort, MAIT cell frequency was decreased in ICU patients compared to IDU patients.

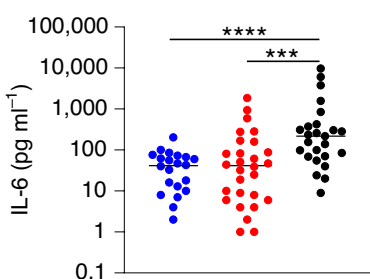

b

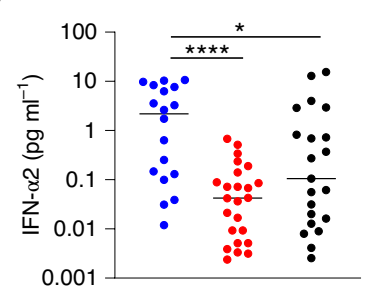

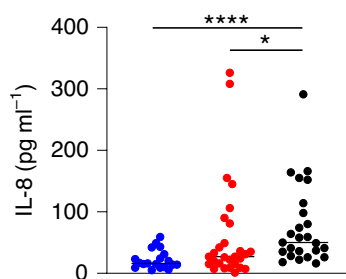
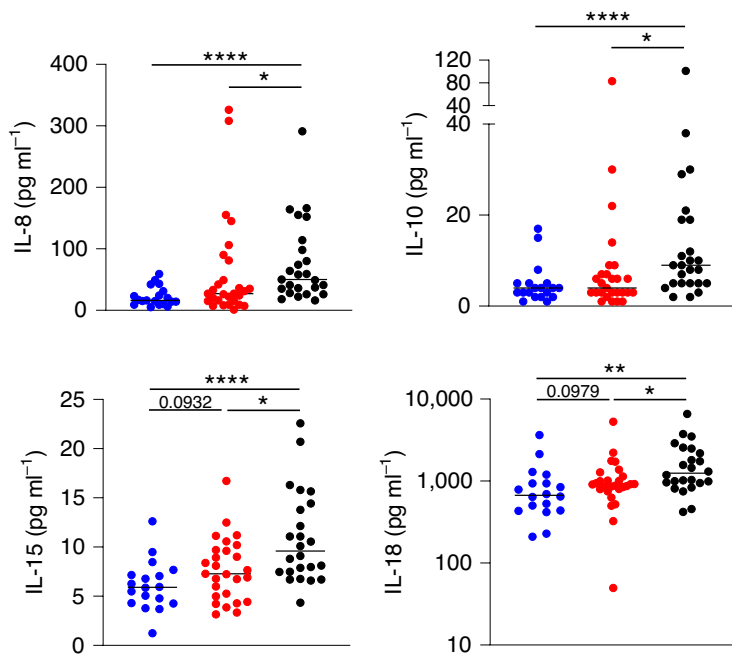

IDU

Deceased

C

ICU surviving

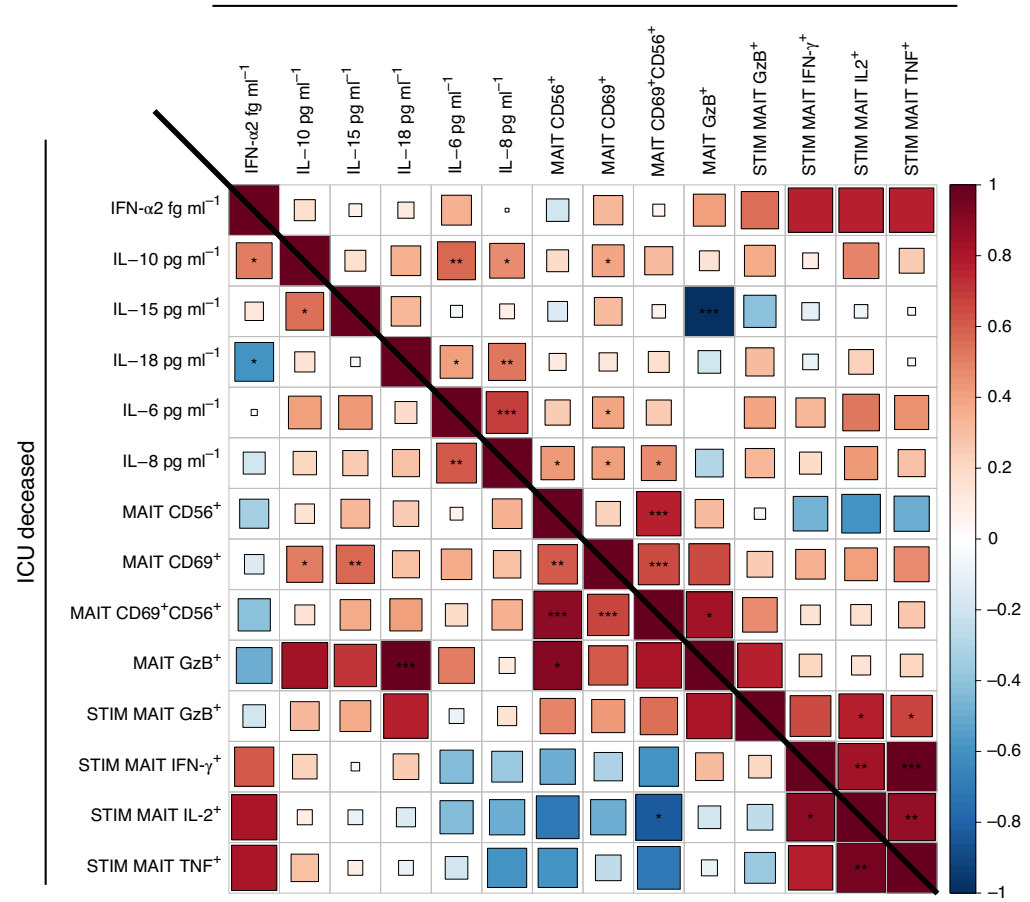

d

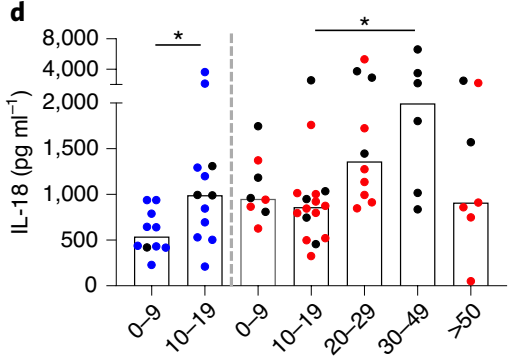

Days after onset of symptoms

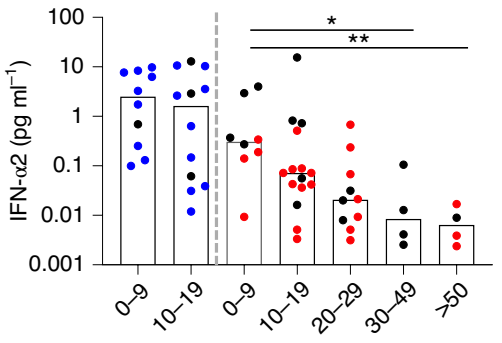

Days after onset of symptoms

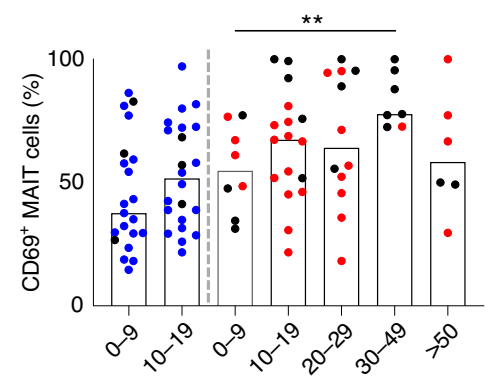

Days after onset of symptoms 
This was associated with a higher expression of CD69 by MAIT cells and an increased production of GzB (Fig. 7a,b). IFN- $\alpha 2$ plasma levels were decreased, whereas IL-15 and IL-18 plasma concentrations were increased in ICU patients compared to IDU patients (Fig. 7c). Blood monocytes were identified (Supplementary Fig. 3) and phenotype analysis showed a nonsignificant decrease in the frequency of $\mathrm{CD} 14^{+} \mathrm{CD} 16^{-}$(classical) monocytes and a significant increase in the frequency of $\mathrm{CD}_{14} 4^{+} \mathrm{CD} 16^{+}$(intermediate) monocytes in ICU patients, as previously described ${ }^{30}$ (Fig. 7d). Correlation studies revealed a strong negative correlation between blood intermediate monocyte frequency and blood MAIT cell frequency in IDU and ICU patients. Conversely, intermediate monocyte frequency correlated positively with GzB-producing MAIT cells (Fig. 7e).

In the first cohort, we observed a progressive decrease in plasma concentrations of IFN- $\alpha 2$ and a progressive increase of plasma IL-18 in ICU patients with COVID-19 during their hospitalization (Fig. 5d). Therefore, we analyzed mRNA levels of IFNA and IL18 in blood monocytes isolated from the second cohort. Monocytes from IDU patients that expressed high levels of IFNA expressed low levels of IL18. Conversely, those expressing low levels of IFNA expressed high levels of IL18. However, all ICU patients expressed higher levels of IL18 than IFNA (Fig. 7f). mRNA levels of STAT1, IRF1 and IRF9, transcription factors involved in IFN- $\alpha 2$ response pathway, were decreased in monocytes from ICU patients. Conversely, mRNA levels of NLRP3 and TXNIP, genes involved in the inflammasome pathway that drives IL18 expression, were increased in monocytes from ICU patients when compared to IDU patients (Fig. $7 \mathrm{~g}, \mathrm{~h}$ ).

Gene expression analysis showed that a short-term in vitro infection of macrophages by SARS-CoV-2 $(24 \mathrm{~h})$ induced upregulation of IFNA, IFNB, IL1B and IL6 expression compared to uninfected controls, whereas IL10, IL15 and IL18 expression was downregulated. Although IFNA, IFNB, ILIB and IL6 expression returned to basal levels $4 \mathrm{~d}$ after infection, the expression of IL10, IL15 and IL18 was upregulated (Fig. 7i).

Taken together, our data suggest that, in severe cases, there is a progressive switch from a type I IFN immune profile in blood toward an IL-18 immune environment in blood through a transcriptional switch in monocytes and macrophages. MAIT cell activation upon infection by SARS-CoV-2 correlates with intermediate monocytes and may be fueled in an early phase through type I IFN response pathways and later through IL-18 response pathways.

SARS-CoV-2-infected macrophages can activate and induce cytotoxicity in MAIT cells. We next assessed the transcriptional profile of blood MAIT cells in uninfected controls $(n=4 ; 13,043$ MAIT cells) and ICU patients ( $n=4 ; 8,832$ MAIT cells) by single-cell RNA sequencing (scRNA-seq) using a droplet-based single-cell platform (10x Chromium). Analysis segregated MAIT cells from uninfected controls and ICU patients, demonstrating a global alteration of their transcriptional activity (Fig. 8a,b). MAIT cells from ICU patients expressed higher levels of genes involved in the inflammatory response (NFKBIA, TXNIP and CD69) but lower levels of IFITM1, a type I IFN-mediated gene associated with antiviral response against coronaviruses ${ }^{31}$. This supports the hypothesis that high MAIT cell activation in severe cases does not rely on type I IFN, as responsive genes are downregulated in MAIT cells (Fig. 8c). scRNA-seq analysis also revealed that cytotoxic response genes such as granulysin (GNLY), PRDM1 (encoding the transcription factor BLIMP-1) and perforin (PRF1) were strongly upregulated in ICU patients compared to uninfected controls (Fig. 8c). Gene expression analysis confirmed that expression of PRDM1, HOBIT and TBX21, three key transcription factors that induce and maintain cellular production of $\mathrm{GzB}^{32}$, was increased in MAIT cells of patients with severe COVID-19 (Fig. 8c).

We next investigated whether macrophages infected with SARS-CoV-2 can directly activate MAIT cells. Blood monocytes isolated from healthy donors were cultured for $7 \mathrm{~d}$ and differentiated into mature granulocyte-macrophage colony-stimulating factor (GM-CSF)-derived macrophages, which were then infected with SARS-CoV-2 at a different multiplicity of infection (MOI; 0.3 or 3 ) for $2 \mathrm{~h}$ and then cocultured with autologous peripheral blood mononuclear cells (PBMCs) for 24-96h (Extended Data Fig. 10a). Infected macrophages from four healthy donors (MOI of 0.3 or 3 ) induced a marked increase in CD69 expression on MAIT cells, when compared to uninfected macrophages (mock), in a dose-dependent manner (Extended Data Fig. 10b). Levels of MAIT cell activation by infected macrophages varied, suggesting intrinsic differences between individuals that modulate MAIT cell response upon infection. This was not dependent of SARS-CoV-2 replication, as the number of viral copies per $10^{5}$ cells was nearly identical in all donors (Extended Data Fig. 10c). Other innate immune cells ( $\gamma \delta$ T and NK cells) were also activated by infected macrophages, however, to a lesser extent than MAIT cells. CD8 and CD4 T cells or ILC3 cocultures (MOI of 3) were almost unaffected (Extended Data Fig. 10d). Analysis of several donors showed that MAIT cells were always activated, whereas this was not the case for other innate immune cells (Extended Data Fig. 10e). Finally, we observed that blockade of type I IFN, IL-12 and IL-18 completely inhibited MAIT cell activation of donor 4, while it was only blocked by type I IFN inhibitor for donor 5 (Extended Data Fig. 10f).

Finally, we examined whether a MAIT cell cytotoxic response could be induced in vitro. When cocultured with macrophages infected with SARS-CoV-2, GzB production by MAIT cells was increased and dampened by anti-MR1 (Fig. 8e). We next addressed whether CD107a, an endosomal marker of cell exocytosis that appears on the cell surface during degranulation and killing ${ }^{33}$, was induced on MAIT cells. In parallel with increased expression of CD69, CD107a was detected on almost all MAIT cells cocultured with macrophages infected with SARS-CoV-2 in an MR1-dependent manner (Fig. $8 \mathrm{f}-\mathrm{h}$ ). These data suggest that infected macrophages

Fig. 6 | MAIT cell phenotype and blood cytokine levels are associated with SARS-CoV-2 infection severity. a-c, Pulmonary damage score level (from 1 (mild) to 5 (critical); a), $\mathrm{PaO}_{2} / \mathrm{FiO}_{2}$ ratio (b) and CRP level (c) of surviving patients with COVID-19 hospitalized in an IDU ( $n=38, n=17$ and $n=44$, respectively) or ICU ( $n=15, n=30$ and $n=27$, respectively) and deceased patients from the IDU and ICU $(n=20, n=27$ and $n=23$, respectively). d, Multiparametric matrix correlation plot of the following clinical data: age, BMI, CRP, SAPS II score, $\mathrm{PaO}_{2} / \mathrm{FiO}_{2}$, PMNs, disease duration and pulmonary lesions score; frequencies of $\mathrm{CD}_{69}{ }^{+}, \mathrm{CD}_{56}{ }^{+}, \mathrm{CD} 69^{+} \mathrm{CD} 56^{+}, \mathrm{CCR} 6^{+} \mathrm{CD} 127^{+}$and $\mathrm{GzB} \mathrm{B}^{+} \mathrm{MAIT}$ cells; frequencies of IFN- $\gamma^{+}, \mathrm{IL}_{-} 2^{+}, \mathrm{TNF}^{+}$and $\mathrm{GzB} \mathrm{B}^{+}$stimulated MAIT cells; in the blood of all patients with COVID-19. Spearman's correlation coefficients are visualized by square size and color intensity. Variables are sorted by hierarchical clustering. e, Correlation between the $\mathrm{PaO}_{2} / \mathrm{FiO}_{2}$ ratio $(n=73)$, SAPS II score $(n=46), \mathrm{CRP}(n=94)$ and PMN $(n=101)$, and the frequency of $\mathrm{CD} 69^{+}$MAIT cells in blood (presented as a percentage of total MAIT cells) from all patients with COVID-19. f,g, PCA of 50 variables (Supplementary Fig. 2), including clinical data and frequencies of immune cell phenotype in patients from the IDU $(n=46)$, ICU $(n=30)$ and in deceased patients from the IDU $(n=6)$ and ICU $(n=21)$. Each point represents a single patient. Mean value of each group is denoted by a larger symbol $(\mathbf{f})$. Arrows represent the contributions of each quantitative variable in the PCA; the first 40 contributing parameters are displayed. Mean values of groups are represented by a symbol. Concentration ellipses with $95 \%$ confidence intervals are shown (g). Small horizontal lines indicate the median. Each symbol represents one patient. ${ }^{*} P<0.05,{ }^{* \star} P<0.01,{ }^{\star \star \star} P<0.001$ and ${ }^{* \star \star *} P<0.0001$ (two-sided Mann-Whitney non-parametric test (a-c) and Spearman's non-parametric correlation test corrected for multiple inferences using Holm's method ( $\mathbf{d}$ and $\mathbf{e})$ ). 
have the ability to induce cytotoxicity of MAIT cells in an MR1-dependent manner. Together, transcriptomic analysis of MAIT cells and in vitro experiments highlight cytotoxic abilities of MAIT cells in the context of SARS-CoV-2 infection.

\section{Discussion}

Our study reveals major MAIT cell alterations in patients with COVID-19. Collapse of blood MAIT cell frequency is associated with high activation and secretion of critical proinflammatory a

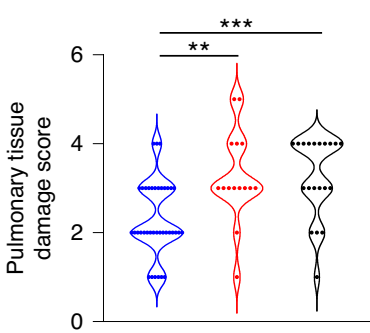

b

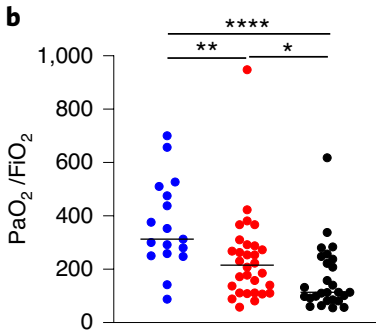

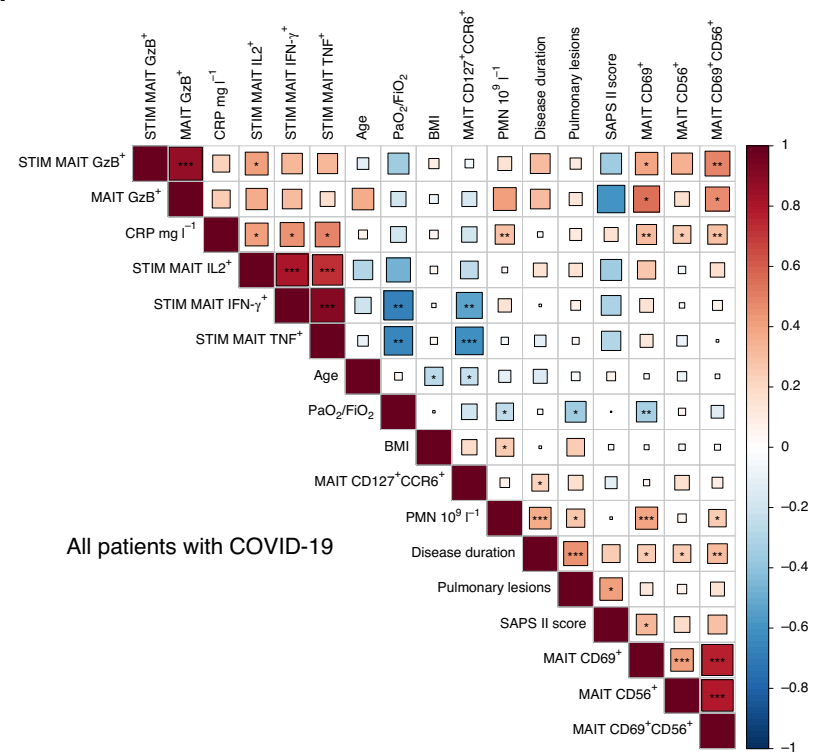

c

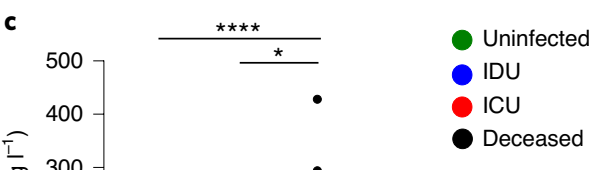

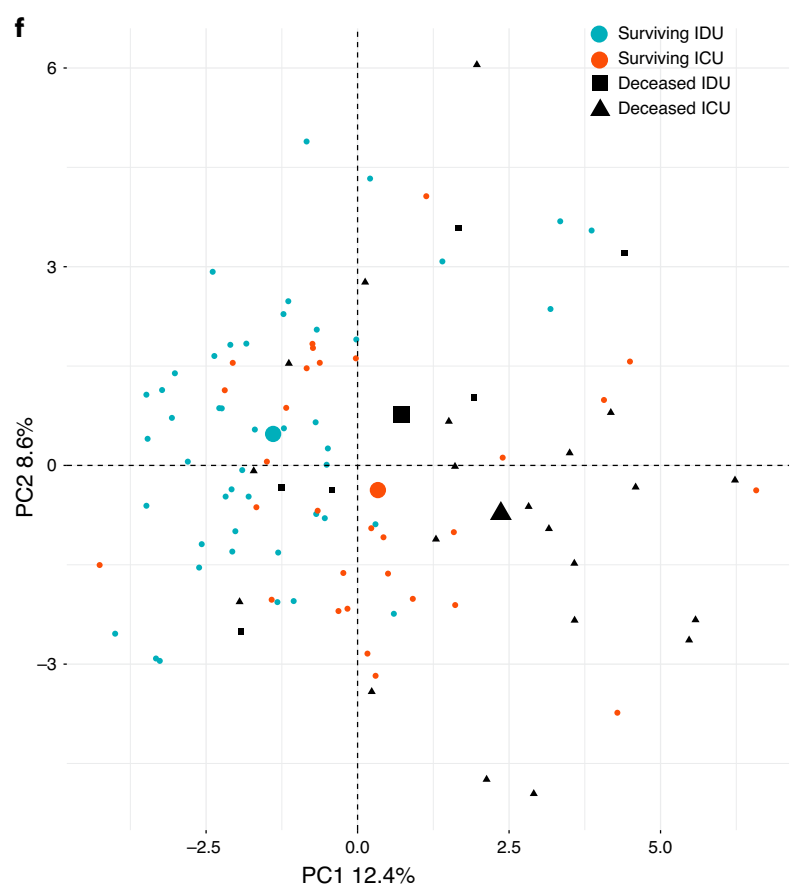

g
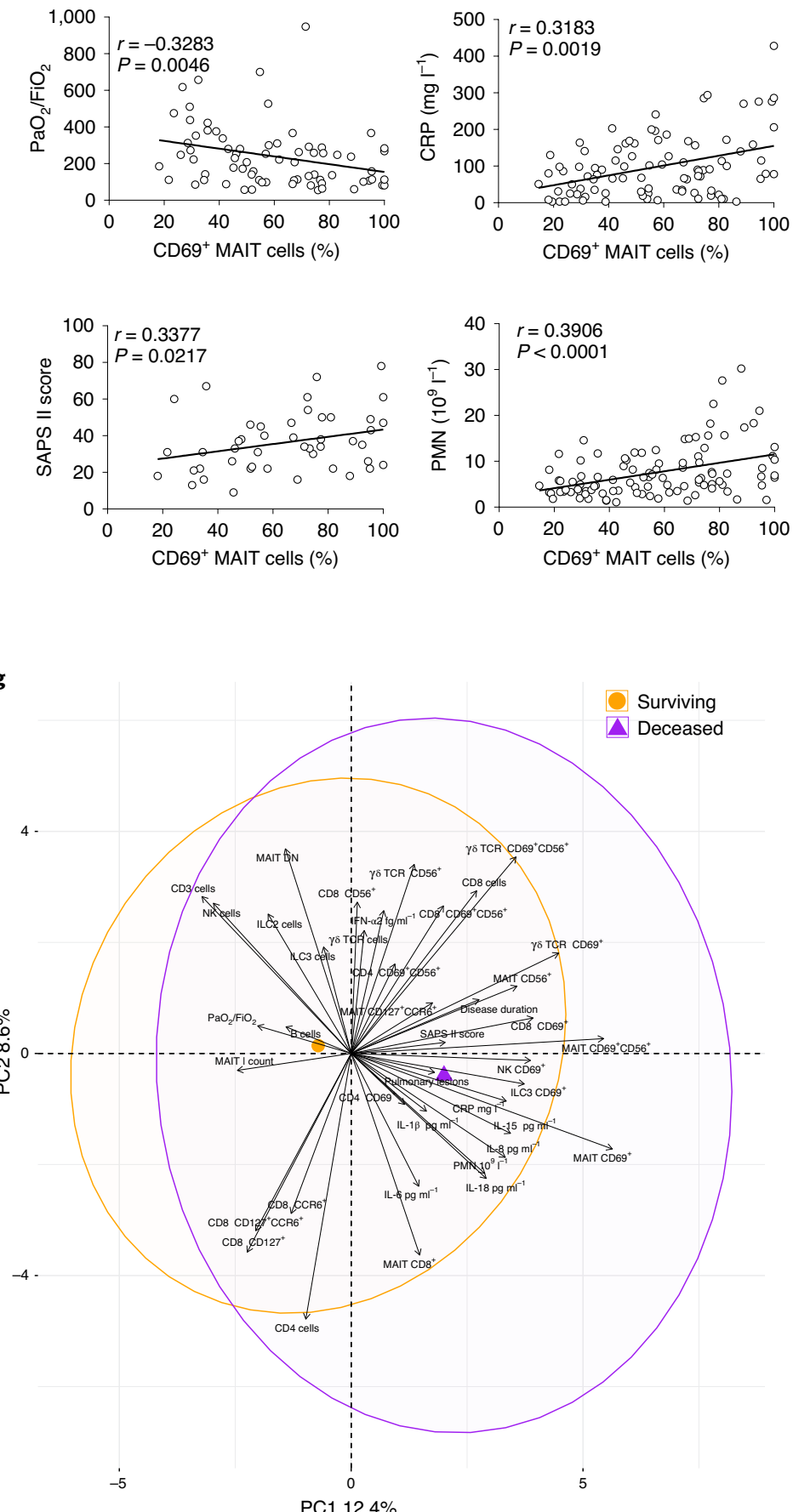
IDU

10x scRNA-seq uninfected

ICU

O 10x scRNA-seq ICU
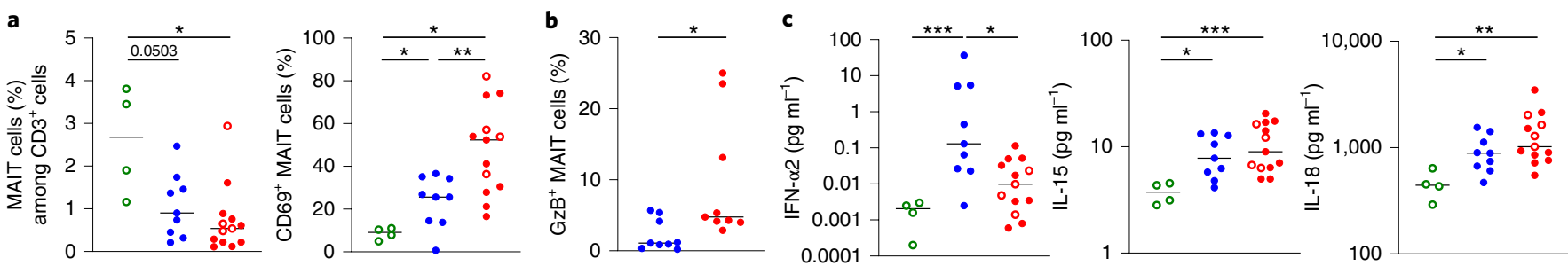

d

Classical monocytes
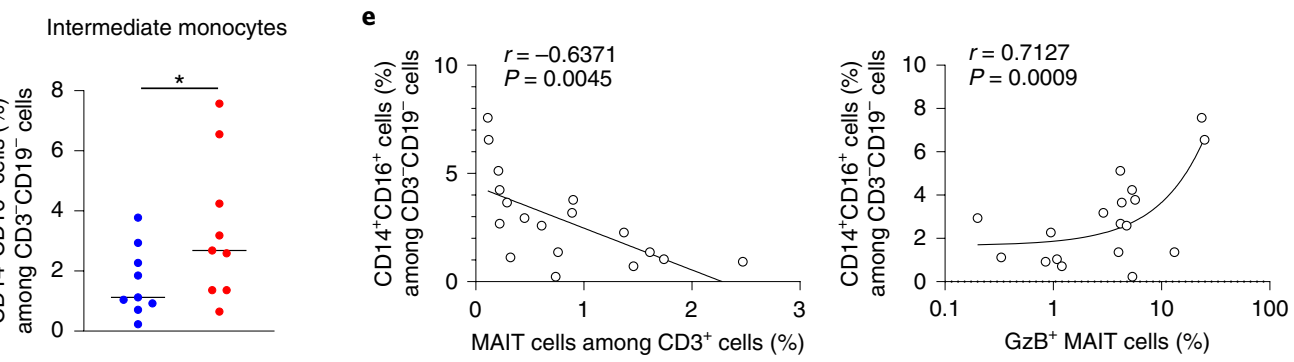

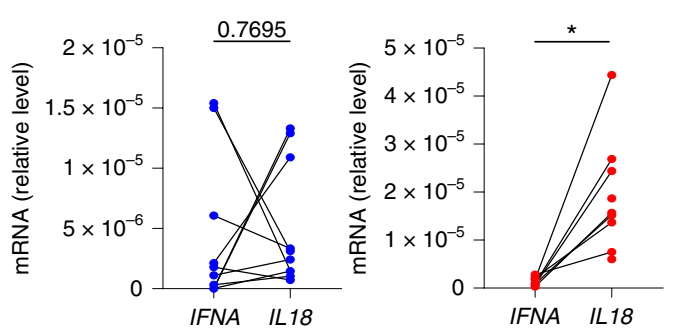

g

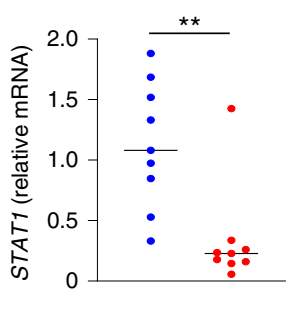

IFN- $\alpha$ pathway

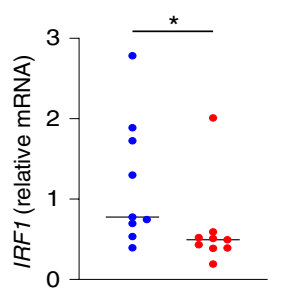

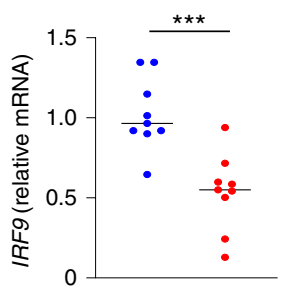
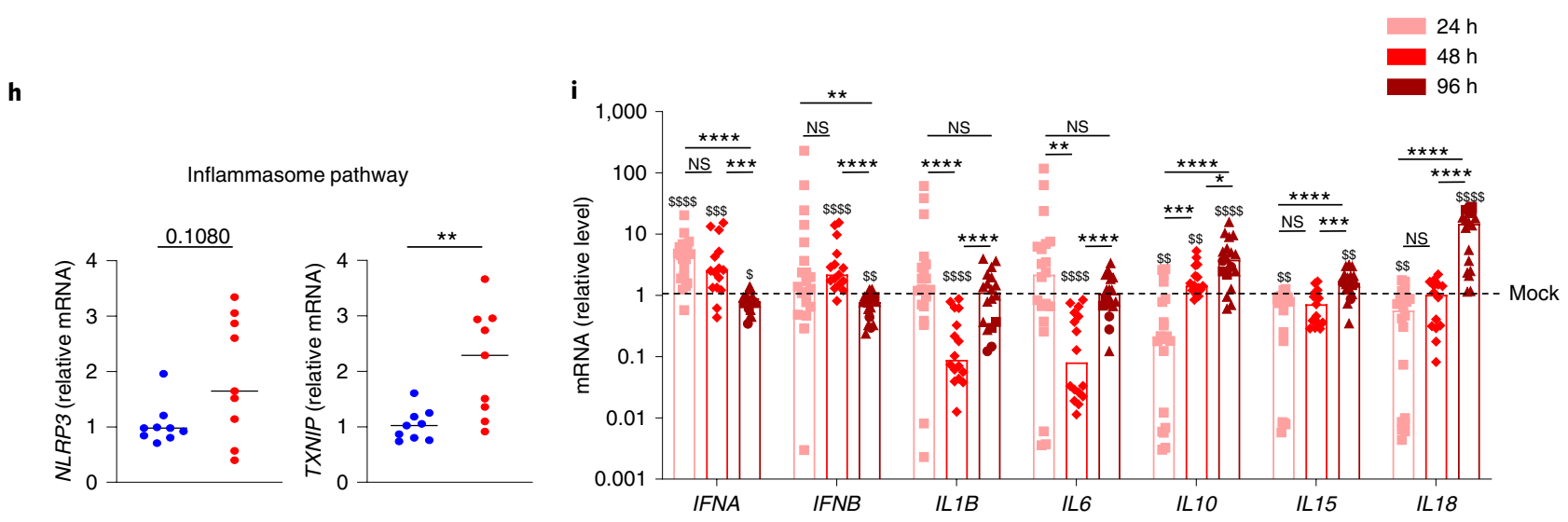

Fig. 7 | MAIT cell phenotype in patients with COVID-19 is associated with a type I IFN to IL-18 monocyte phenotype switch. a, Flow cytometry analysis of MAIT cells and CD69+ MAIT cells from blood of uninfected controls $(n=4)$ and patients with COVID-19 admitted to an IDU $(n=9)$ or ICU $(n=9)$. $\mathbf{b}$, Flow cytometry analysis of $\mathrm{GzB}^{+}$MAIT cell frequency from blood of patients with COVID-19 admitted to an IDU $(n=9)$ or ICU $(n=9)$. c, Cytokine quantification by electrochemiluminescence immunoassay of IFN- $\alpha 2$, IL-15 and IL-18 in the plasma of uninfected patients $(n=4)$ and patients with COVID-19 in the IDU $(n=9)$ or ICU $(n=13)$. Empty symbols represent patients who had MAIT cells sequenced using Chromium 10x technology. $\mathbf{d}$, Flow cytometry analysis of circulating $\mathrm{CD}_{14}{ }^{+} \mathrm{CD} 16-$ (classical) monocytes and $\mathrm{CD} 14{ }^{+} \mathrm{CD} 16+$ (intermediate) monocyte frequencies from patients with COVID-19 who were admitted to an IDU $(n=9)$ or ICU $(n=9)$. e, Correlation between MAIT cell frequencies or GzB+ MAIT cell frequencies (presented as a percentage of total MAIT cells) and $\mathrm{CD} 14^{+} \mathrm{CD} 16^{+}$intermediate monocyte frequency $(n=18)$ in the blood of patients with COVID-19. f, Relative mRNA levels of IFNA and IL18 in sorted $\mathrm{CD}_{14}^{+}$monocytes from blood of patients with COVID-19 admitted to an IDU $(n=9)$ or ICU $(n=9)$. $\mathbf{g}$, Relative mRNA levels of STAT1, IRF1 and IRF9 in sorted CD14+ monocytes from blood of patients with COVID-19 admitted to an IDU $(n=9)$ or ICU $(n=9)$. $\mathbf{h}$, Relative mRNA levels of NLRP3 and TXNIP in sorted CD14+ monocytes from blood of patients admitted to an IDU $(n=9)$ or ICU $(n=9)$. i, Kinetics of IFNA, IFNB, IL1B, IL6, IL7, IL10, IL15 and IL18 mRNA relative levels in SARS-CoV-2-infected macrophages from six healthy donors at $24 \mathrm{~h}, 48 \mathrm{~h}$ and $96 \mathrm{~h}$ after infection $(n=3-4$ replicates per donor). $\$$ symbol represents significant differences from the respective mock. Results from quantitative PCR with reverse transcription (RT-qPCR) were normalized to the expression of the reference housekeeping $18 S$ gene $(\mathbf{f}-\mathbf{i})$ and normalized on mock-infected macrophages (i). Small horizontal lines indicate the median. Each symbol represents one patient (a-h) or one replicate (i). ${ }^{*} \$, P<0.05 ;{ }^{* \star} / \$ \$, P<0.01 ;{ }^{* \star \star} / \$ \$ \$, P<0.001 ;{ }^{\star * \star \star} / \$ \$ \$ \$, P<0.0001$ (two-sided MannWhitney non-parametric test (a-d and $\mathbf{g - i}$ ) and Spearman's non-parametric correlation test (e) and Wilcoxon signed-rank test (f)). NS, not significant. 
a

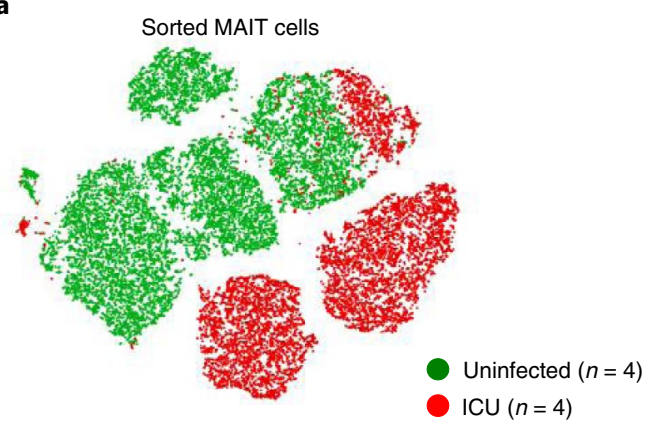

b

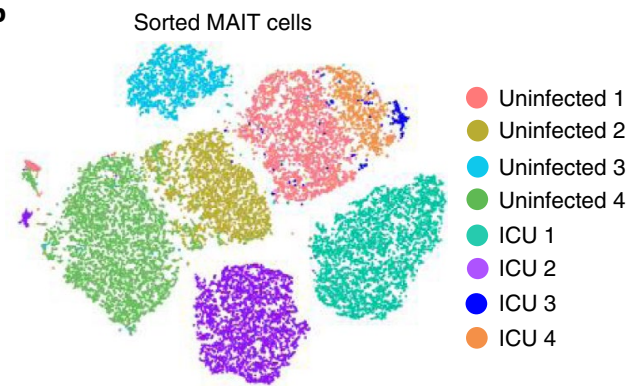

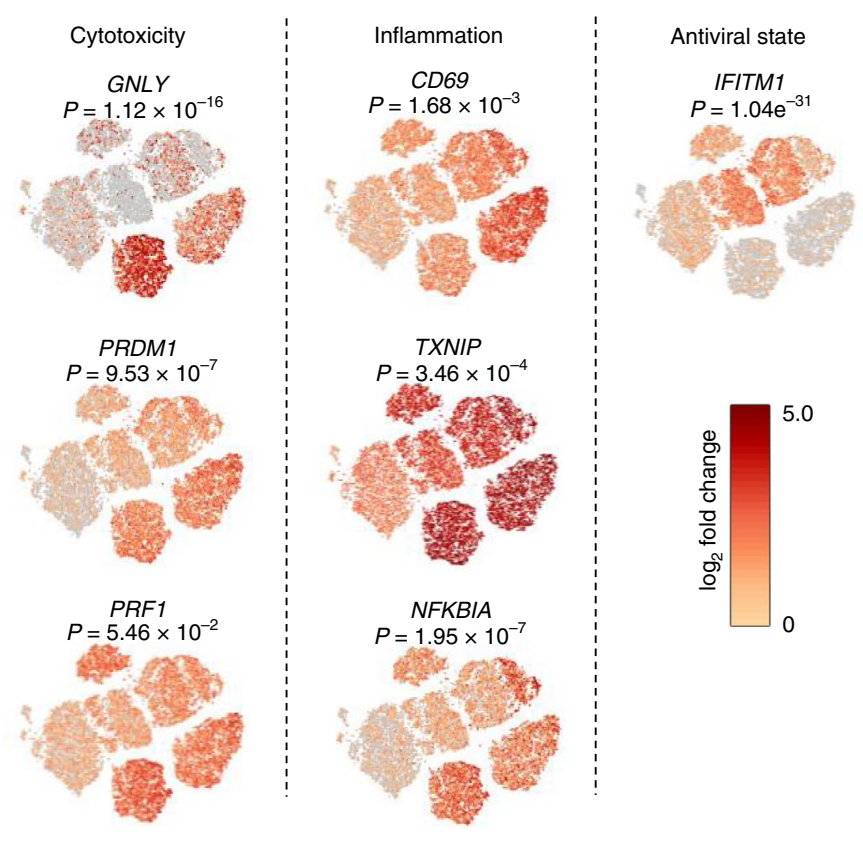

d

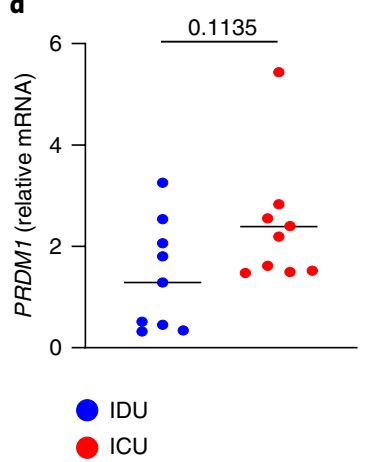

GrB pathway

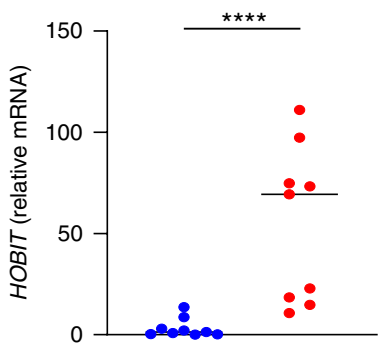

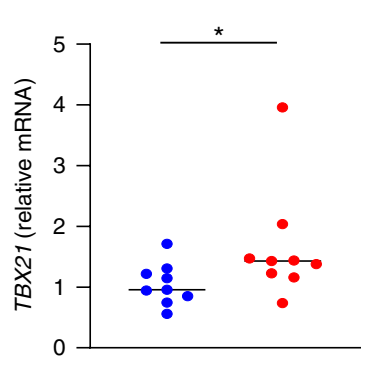

f

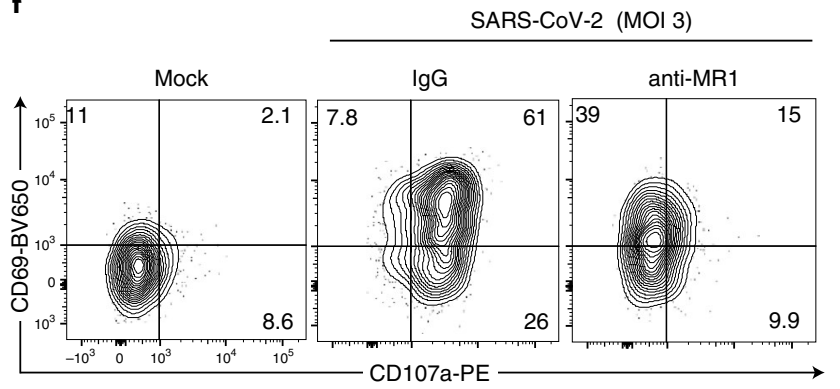

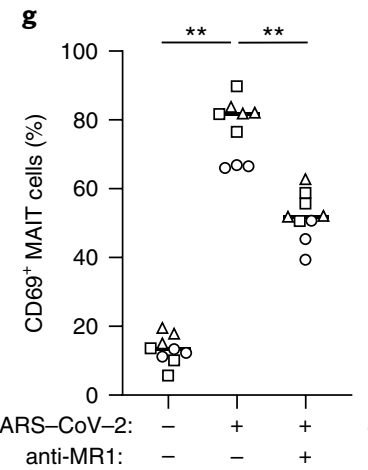

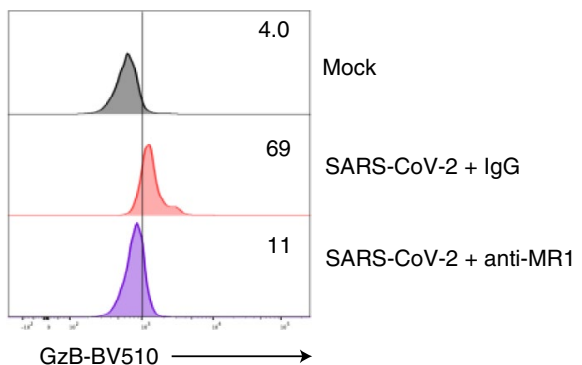

GzB-BV510

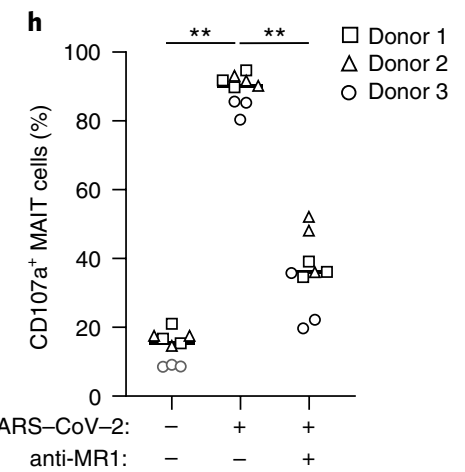

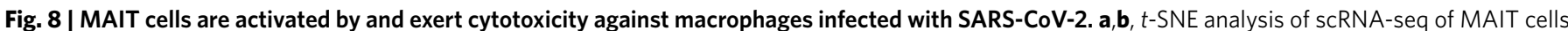

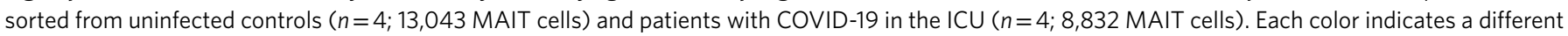
condition (uninfected or ICU; a) or a unique patient (b). c, $t$-SNE analysis representing the log fold change in the expression of GNLY, PRDM1, PRF1, CD69, TXNIP, NFKBIA and IFITM1 between uninfected controls and patients with COVID-19 in the ICU ( $n=4$ for each group). d, Relative mRNA levels of PRDM1, HOBIT and TBX21 from sorted CD161+Va7.2+ MAIT cells from patients with COVID-19 admitted to an IDU ( $n=9)$ and ICU ( $n=9$ ). e, Representative mean fluorescence intensity of GzB expression in MAIT cells from one healthy donor, cocultured for $4 \mathrm{~d}$ with either uninfected or SARS-CoV-2-infected macrophages (MOI of 3) and supplemented or not with anti-MR1. f-h, Representative quantile contour plots and frequencies of CD69+ and CD107a+

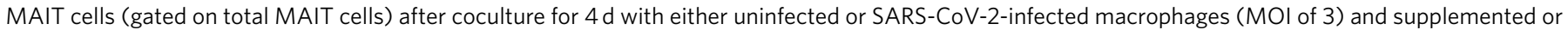

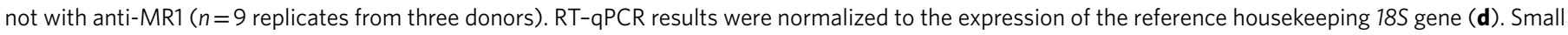
horizontal lines indicate the median. Each symbol represents one patient (d) or one replicate $(\mathbf{g}$ and $\mathbf{h})$. ${ }^{\star} P<0.05$, ${ }^{\star \star} P<0.01$ and ${ }^{\star \star \star \star} P<0.0001$ (two-sided Mann-Whitney non-parametric test (d) and Wilcoxon signed-rank test ( $\mathbf{g}$ and $\mathbf{h})$ ). 
cytokines and GzB. MAIT cell GzB production is enhanced in lung fluids as compared to those in blood. Moreover, all blood MAIT cell alterations scale with the severity of SARS-CoV-2 infection, and correlate with plasmatic proinflammatory cytokine levels and other innate cell activation. We also identified a SARS-CoV-2 monocyte/ macrophage cytokine shift and the ability of infected macrophages to induce cytotoxicity of MAIT cells.

The frequency of blood MAIT cells was reduced down to a tenth compared to controls in severe cases of COVID-19 (refs. ${ }^{25,26}$ ). The tissue migration chemokine receptor CCR6 expression is decreased on blood MAIT cells during SARS-CoV-2 infection, whereas its ligand, CCL20, is expressed by proinflammatory pulmonary macrophages ${ }^{34}$. Collapse of blood MAIT cell frequency could reflect migration of CCR6 ${ }^{+}$MAIT cells in the infected lung where they might participate in local immune response and tissue damage. Indeed, we observed an increased frequency of total and cytotoxic MAIT cells in lung fluids compared to blood in patients with severe disease. Low blood MAIT cell frequency may also be due to apoptosis. Expression of CD127, the receptor for the prosurvival cytokine IL-7, is reduced on blood MAIT cells from infected patients.

MAIT cell frequency and activation alterations are characteristic of several deleterious inflammatory pathologies, especially metabolic pathologies such as diabetes, liver disease or obesity ${ }^{9,35-38}$. MAIT cell frequency is reduced in blood from patients with those pathologies and they present an exhausted phenotype that may impair their antibacterial response $\mathrm{e}^{36-38}$. This is of importance as metabolic pathologies are important factors increasing the risk of developing severe COVID-19 (refs. ${ }^{39-42}$ ). Thus, we matched controls for comorbidities affecting patients with COVID-19. More than half of ICU patients in our first cohort developed secondary infections, from mild tissue-specific infections to life-threatening septic shock. High frequency of MAIT cells is associated with survival during septic shock ${ }^{43}$. Patients with COVID-19 who have metabolic syndromes may therefore be more vulnerable to complications because of a diminished ability to fight primary viral and secondary bacterial infections due to a depletion of blood MAIT cells.

Blood cytokine analysis showed increased amounts of type I IFN and proinflammatory cytokines such as IL-6, IL- 8 and the immunosuppressive IL-10 in patients with COVID-19, as previously reported ${ }^{27}$. We show that IL-15 and IL-18 levels were also increased in patients with COVID-19 in relation to disease severity, and IL-18 strongly correlated with MAIT cell activation in patients with fatal outcomes. In these patients, IFN- $\alpha 2$ level inversely correlated with increased IL-18. Such cytokine imbalance was also observed in monocytes from IDU and ICU patients. During early phases of in vitro infection, macrophages produced important amounts of type I IFN that collapsed around $4 \mathrm{~d}$ after infection. This is in agreement with the ability of SARS-CoV-2 to suppress type I IFN production through structural and nonstructural viral proteins ${ }^{44}$ and as highlighted in plasma of ICU patients with COVID-19. Transcriptional analysis of monocytes showed that type I IFN-responsive transcription factors were downregulated in ICU patients, whereas the NLRP3 inflammasome pathway was upregulated, in line with increased expression of IL-18. Analysis of MAIT cells in the same patients showed a strong correlation between MAIT cell GzB production and the $\mathrm{CD} 14^{+} \mathrm{CD} 16^{+}$monocyte subset that is increased in patients with severe disease $e^{23,24,30}$.

Our study highlights the cytotoxic potential of MAIT cells in patients with COVID-19. There was an elevated frequency of MAIT cells producing GzB in blood and pulmonary fluids. Blood MAIT cell GzB expression was one of the parameters most associated with a fatal outcome. Single-cell and bulk transcriptomic analysis of MAIT cells further showed elevated expression of several cytotoxic genes and master transcriptional factors of the GzB pathway. In vitro data show that MAIT cells degranulated in an
MR1-dependent manner in the presence of infected macrophages. GzB expression by MAIT cells is strongly linked to a fatal outcome in severe patients, which is not the case for conventional CD8 T cells. Because MR1 is ubiquitous and upregulated upon inflammation ${ }^{45}$, MAIT cells might induce tissue damage by broad killing in contrast to conventional CD8 $\mathrm{T}$ cells that only kill infected cells presenting viral epitopes. Even though in vitro experiments were performed without adding exogenous MAIT cell ligand, an altered microbiome in affected tissue ${ }^{46}$ and in the host at large may modulate MAIT cell activity through the production of MR1-restricted ligands. A recent study showed an enhanced level of bacterial DNA and lipopolysaccharide in the plasma of patients with severe COVID-19 (ref. ${ }^{47}$ ). Similarly, bacterial MAIT cell ligand levels could be increased and participate in systemic MAIT cell activation.

MAIT cells in inflammatory pathologies are double-edged swords. They can be protective by participating in pathogen clearance, immune activation and tissue reparation ${ }^{9,11,12,16,18}$. Conversely, MAIT cells may participate in the deleterious inflammation affecting severe, long-term hospitalized patients with COVID-19. Indeed, the immune response is at this stage in a second proinflammatory phase more detrimental than the viral infection per se. Our data suggest a negative role for MAIT cells in severe COVID-19 infection in which their activation and GzB production are at the highest levels. Against infection with dengue virus (DV), there is a temporal and quantitative association between activation of MAIT cells and onset of severe disease ${ }^{18}$. Our data in patients with COVID-19 are reminiscent of this deleterious role of MAIT cells in patients infected with DV that differ from their suggested protective role in patients with influenza. Interestingly, DV and SARS-CoV-2 are able to infect macrophages, which then can activate MAIT cells ${ }^{48}$. Activation of MAIT cells by infected macrophages through IL-18 may switch MAIT cells toward a similar detrimental role in these infections.

In conclusion, human MAIT cells are activated, displaying a cytotoxic profile in the blood and lung of patients with SARS-CoV-2 infection, which is associated with other innate immune cell activation and a proinflammatory environment. Together, these data extend the knowledge of the immune actors involved during SARS-CoV-2 infection. These findings reveal MAIT cells as a valuable biomarker of disease progression and as a new target for interventional therapeutic approaches in severe SARS-CoV-2 infection.

\section{Online content}

Any methods, additional references, Nature Research reporting summaries, source data, extended data, supplementary information, acknowledgements, peer review information; details of author contributions and competing interests; and statements of data and code availability are available at https://doi.org/10.1038/ s41590-021-00870-z.

Received: 25 August 2020; Accepted: 11 January 2021; Published online: 2 February 2021

\section{References}

1. Wrapp, D. et al. Cryo-EM structure of the 2019-nCoV spike in the prefusion conformation. Science 367, 1260-1263 (2020).

2. Tay, M. Z., Poh, C. M., Rénia, L., MacAry, P. A. \& Ng, L. F. P. The trinity of COVID-19: immunity, inflammation and intervention. Nat. Rev. Immunol. https://doi.org/10.1038/s41577-020-0311-8 (2020).

3. Chen, N. et al. Epidemiological and clinical characteristics of 99 cases of 2019 novel coronavirus pneumonia in Wuhan, China: a descriptive study. Lancet 395, 507-513 (2020)

4. Huang, C. et al. Clinical features of patients infected with 2019 novel coronavirus in Wuhan, China. Lancet 395, 497-506 (2020).

5. Thompson, B. T., Chambers, R. C. \& Liu, K. D. Acute respiratory distress syndrome. N. Engl. J. Med. 377, 562-572 (2017).

6. Matthay, M. A. et al. Acute respiratory distress syndrome. Nat. Rev. Dis. Primers 5, 18 (2019). 
7. Gorbalenya, A. E. et al. The species severe acute respiratory syndrome-related coronavirus: classifying 2019-nCoV and naming it SARS-CoV-2. Nat. Microbiol. 5, 536-544 (2020).

8. McCarthy, N. E. \& Eberl, M. Human $\gamma \delta$ T cell control of mucosal immunity and inflammation. Front. Immunol. 9, 985 (2018).

9. Toubal, A., Nel, I., Lotersztajn, S. \& Lehuen, A. Mucosal-associated invariant T cells and disease. Nat. Rev. Immunol. 19, 643-657 (2019).

10. Trottein, F. \& Paget, C. Natural killer T cells and mucosal-associated invariant T cells in lung infections. Front Immunol. 9, 1750 (2018).

11. Constantinides, M. G. et al. MAIT cells are imprinted by the microbiota in early life and promote tissue repair. Science $\mathbf{3 6 6}$, eaax6624 (2019).

12. Leng, T. et al. TCR and inflammatory signals tune human MAIT cells to exert specific tissue repair and effector functions. Cell Rep. 28, 3077-3091 (2019).

13. Stehle, C., Hernández, D. C. \& Romagnani, C. Innate lymphoid cells in lung infection and immunity. Immunol. Rev. 286, 102-119 (2018).

14. Treiner, E. et al. Selection of evolutionarily conserved mucosal-associated invariant T cells by MR1. Nature 422, 164-169 (2003).

15. Corbett, A. J. et al. T cell activation by transitory neo-antigens derived from distinct microbial pathways. Nature 509, 361-365 (2014)

16. Loh, L. et al. Human mucosal-associated invariant $\mathrm{T}$ cells contribute to antiviral influenza immunity via IL-18-dependent activation. Proc. Natl Acad. Sci. USA 113, 10133-10138 (2016).

17. Ussher, J. E., Willberg, C. B. \& Klenerman, P. MAIT cells and viruses. Immunol. Cell Biol. 96, 630-641 (2018).

18. van Wilgenburg, B. et al. MAIT cells are activated during human viral infections. Nat. Commun. 7, 11653 (2016).

19. Ussher, J. E. et al. CD161 ${ }^{++} \mathrm{CD}^{+} \mathrm{T}$ cells, including the MAIT cell subset, are specifically activated by IL-12+ IL-18 in a TCR-independent manner. Eur. J. Immunol. 44, 195-203 (2014).

20. Leeansyah, E. et al. Activation, exhaustion and persistent decline of the antimicrobial MR1-restricted MAIT cell population in chronic HIV-1 infection. Blood 121, 1124-1135 (2013).

21. Leeansyah, E. et al. Arming of MAIT cell cytolytic antimicrobial activity is induced by IL-7 and defective in HIV-1 infection. PLoS Pathog. 11, e1005072 (2015)

22. Wang, F. et al. Characteristics of peripheral lymphocyte subset alteration in COVID-19 pneumonia. J. Infect. Dis. 221, 1762-1769 (2020).

23. Silvin, A. et al. Elevated calprotectin and abnormal myeloid cell subsets discriminate severe from mild COVID-19. Cell https://doi.org/10.1016/j cell.2020.08.002 (2020)

24. Schulte-Schrepping, J. et al. Severe COVID-19 is marked by a dysregulated myeloid cell compartment. Cell https://doi.org/10.1016/j.cell.2020.08.001 (2020)

25. Jouan, Y. et al. Phenotypical and functional alteration of unconventional T cells in severe COVID-19 patients. J. Exp. Med. 217, e20200872 (2020).

26. Parrot, T. et al. MAIT cell activation and dynamics associated with COVID-19 disease severity. Sci. Immunol. 5, eabe1670 (2020).

27. Hadjadj, J. et al. Impaired type I interferon activity and inflammatory responses in severe COVID-19 patients. Science https://doi.org/10.1126/ science.abc6027 (2020).

28. Zhang, J.-Y. et al. Single-cell landscape of immunological responses in patients with COVID-19. Nat. Immunol. https://doi.org/10.1038/s41590-0200762-x (2020)
29. Merad, M. \& Martin, J. C. Pathological inflammation in patients with COVID-19: a key role for monocytes and macrophages. Nat. Rev. Immunol. 20, 355-362 (2020).

30. Zhou, Y. et al. Pathogenic T cells and inflammatory monocytes incite inflammatory storms in severe COVID-19 patients. Natl Sci. Rev. 7, 998-1002 (2020).

31. Huang, I.-C. et al. Distinct patterns of IFITM-mediated restriction of filoviruses, SARS coronavirus and influenza A virus. PLoS Pathog. 7, e1001258 (2011).

32. Kragten, N. A. M. et al. Blimp-1 induces and Hobit maintains the cytotoxic mediator granzyme B in CD8 T cells. Eur. J. Immunol. 48, 1644-1662 (2018).

33. Kurioka, A. et al. MAIT cells are licensed through granzyme exchange to kill bacterially sensitized targets. Mucosal Immunol. 8, 429-440 (2015).

34. Chua, R. L. et al. COVID-19 severity correlates with airway epitheliumimmune cell interactions identified by single-cell analysis. Nat. Biotechnol. 38, 970-979 (2020)

35. Hegde, $\mathrm{P}$. et al. Mucosal-associated invariant $\mathrm{T}$ cells are a profibrogenic immune cell population in the liver. Nat. Commun. 9, 2146 (2018).

36. Rouxel, O. et al. Cytotoxic and regulatory roles of mucosal-associated invariant T cells in type 1 diabetes. Nat. Immunol. 18, 1321-1331 (2017).

37. Magalhaes, I. et al. Mucosal-associated invariant T cell alterations in obese and type 2 diabetic patients. J. Clin. Invest. 125, 1752-1762 (2015).

38. Toubal, A. et al. Mucosal-associated invariant T cells promote inflammation and intestinal dysbiosis leading to metabolic dysfunction during obesity. Nat. Commun. 11, 3755 (2020).

39. Zhou, F. et al. Clinical course and risk factors for mortality of adult inpatients with COVID-19 in Wuhan, China: a retrospective cohort study. Lancet 395 , 1054-1062 (2020).

40. Cariou, B. et al. Phenotypic characteristics and prognosis of inpatients with COVID-19 and diabetes: the CORONADO study. Diabetologia 63, 1500-1515 (2020).

41. Lisco, G. et al. Hypothesized mechanisms explaining poor prognosis in type 2 diabetes patients with COVID-19: a review. Endocrine https://doi.org/10.1007/ s12020-020-02444-9 (2020).

42. Czernichow, S. et al. Obesity doubles mortality in patients hospitalized for SARS-CoV-2 in Paris hospitals, France: a cohort study on 5,795 patients. Obesity https://doi.org/10.1002/oby.23014 (2020).

43. Grimaldi, D. et al. Specific MAIT cell behaviour among innate-like T lymphocytes in critically ill patients with severe infections. Intensive Care Med. 40, 192-201 (2014).

44. Lei, X. et al. Activation and evasion of type I interferon responses by SARS-CoV-2. Nat. Commun. 11, 3810 (2020).

45. Riegert, P., Wanner, V. \& Bahram, S. Genomics, isoforms, expression and phylogeny of the MHC class I-related MR1 gene. J. Immunol. 161, 4066-4077 (1998).

46. Fan, J. et al. The lung tissue microbiota features of 20 deceased patients with COVID-19. J. Infect. 81, e64-e67 (2020).

47. Arunachalam, P. S. et al. Systems biological assessment of immunity to mild versus severe COVID-19 infection in humans. Science 369, 1210-1220 (2020).

48. Blackley, S. et al. Primary human splenic macrophages, but not T or B cells, are the principal target cells for dengue virus infection in vitro. J. Virol. 81, 13325-13334 (2007).

Publisher's note Springer Nature remains neutral with regard to jurisdictional claims in published maps and institutional affiliations.

(c) The Author(s), under exclusive licence to Springer Nature America, Inc. 2021 


\section{Methods}

Clinical study design and ethical statements. A first cohort of 102 patients with COVID-19 admitted to Bichat or Cochin hospitals (Paris, France) between 23 March 2020 and 29 May 2020 was included in this clinical study. A second, small cohort of 22 patients with COVID-19 admitted to Bichat or Cochin hospitals between 24 September 2020 and 5 October 2020 was also included in this clinical study. For comparisons, blood from 84 uninfected controls of the QUID-NASH project $(n=30)$, Etablissement Français du Sang $(n=12)$ and volunteer donors $(n=42)$ were mostly collected before the onset of the pandemic ( 54 of 84$)$. Clinical characteristics of the 182 patients from cohort 1 and 26 patients from cohort 2 are summarized in Extended Data Figs. 1 and 9 and Supplementary Table 1. No statistical methods were used to predetermine cohort size. The percentage of lung involvement was evaluated on chest computed tomography, with a score of 1 to 5 representing mild to critical pulmonary lesion damage. SAPS II was used to estimate the probability of survival after ICU admission. The ethics committees (detailed below) approved clinical investigations, and informed consent was obtained from each enrolled patient or from their next of kin, in accordance with national laws. Patient's from Bichat Hospital were included in the French COVID-19 cohort (NCT04262921). Ethics approval for this cohort was granted on 5 February 2020 by the French Ethics Committee Comité de Protection des Personnes (CPP)-Ile de France-VI (2020-A00256-33). This cohort is sponsored by Inserm and supported by the REACTing consortium and by the French Ministry of Health (PHRC, 20-0424). Samples from these patients were derived from samples collected in routine care. For lung fluid samples from Bichat hospital, samples were obtained under authorization from the ethics committee CPP-Ile de France-XI (2020-001457643, 20037-25957 and APHP 200388-COVIDICUS). Other lung fluid samples were obtained from the Regional University Tours Hospital (NCT03379207) and under authorization from the ethics committee CPP-Ile-de-France-VIII (2017-A01841-52). Patients from Cochin Hospital were recruited in the setting of the local RADIPEM biological samples collection derived from samples collected in routine care. Biological collection and informed consent were approved by the Direction de la Recherche Clinique et Innovation and the French Ministry of Research (2019-3677). Investigations with control patients from QUID-NASH were approved by CPP Sud Méditerranée V (18.021; QUID project registration no. 2018-A00311-54)

\section{Whole blood, plasma isolation, peripheral blood mononuclear cells,} endotracheal aspirates and bronchoalveolar lavage. Whole blood samples were collected in EDTA- or heparin-coated tubes (Vacutaine, BD Biosciences) from healthy, uninfected donors, and patients with COVID-19 admitted to Cochin, Bichat or Lariboisière Hospitals. Tubes were centrifugated at $1,260 \mathrm{~g}$ for $10 \mathrm{~min}$ and plasma was collected and frozen at $-80^{\circ} \mathrm{C}$. PBMCs were isolated by Ficoll-Paque (Lymphosep, Biosera), frozen in $1 \mathrm{ml}$ of freezing medium comprising $90 \%$ FCS and $10 \%$ dimethylsulfoxide and stored in liquid nitrogen.

ETA and BAL samples were collected from patients with COVID-19 who were intubated $(n=10)$ from Bichat and Tours hospitals. Patients were under invasive mechanical ventilation. Samples were weighted and incubated in PBS $\left(5 \mathrm{ml} \mathrm{g}^{-1}\right)$ in the presence of $1 \mu \mathrm{M}$ DL-dithiothreitol (D5545, Sigma) for $30 \mathrm{~min}$ at $4^{\circ} \mathrm{C}$ under gentle agitation for cellular dispersion. After centrifugation, cell pellets were filtered through a $100-\mu \mathrm{m}$ cell strainer. Red blood cells were removed using a red blood cell lysis buffer and then cells were filtered through a $40-\mu \mathrm{m}$ cell strainer before staining for flow cytometry.

Cytokine measurements. IL-6, IL-8 and IL-10 levels were measured in plasma using human CBA Inflammatory Cytokine Kit (BD Biosciences) according to the manufacturer's instructions. Acquisitions were performed on a BD FACSLyric cytometer (BD Biosciences) and raw data analyzed with FCAP Array software v3.0 (BD Biosciences). For the IFN- $\alpha 2$ quantification, plasma samples were analyzed with the MSD Quickplex using the ultra-sensitive assay S-PLEX human IFN- $\alpha 2 \mathrm{a}$ (K151P3S-1, Meso Scale Diagnostic) using $25 \mu \mathrm{l}$ of each sample. Each plasma sample was assayed twice with the average value taken as the final result.

For IL-18, IL-15 and IL-1 $\beta$ quantification, the plasma samples were analyzed with the MSD Quickplex using the U-plex Biomarker group 1 (human) assay (K-15067L-1, Meso Scale Diagnostic) using $25 \mu$ l of each (1/2 diluted) sample. Each plasma sample was assayed twice with the average value taken as the final result. The units for human IFN- $\alpha 2 \mathrm{a}, \mathrm{IL}-18$, IL-15 and IL- $1 \beta$ measured in the present study were $\mathrm{pg} \mathrm{ml}^{-1}$.

Flow cytometry. Surface and intracytoplasmic staining were performed on blood samples or PBMCs with the following antibodies: CD3 (OKT3), CD19 (HIB19), CD4 (OKT4), CD8 (SK1), V $\alpha 7.2$ (3C10), TCR $\gamma \delta(B 1)$, CD161 (HP-3G10), CCR6 (G034E3), CD25 (M-A251), CD56 (HCD56), CD69 (FN50), CD127 (R34.34), CD218a (H44), CD107a (eBioH4A3), CD11b (CBRM1/5), CD14 (M5E2), CD16 (CB16), IFN- $\gamma$ (4S.B3), TNF (Mab11), IL-2 (MQ1-17H12), IL-4 (8D4-8), IL-10 (JES3-19F1), IL-17 (BL168) and GzB (GB11). A full list is described in Supplementary Table 1. According to the amount of blood obtained from each patient, surface staining was always performed, and depending on the number of cells, intracytoplasmic staining of cytokines and $\mathrm{GzB}$ was analyzed after stimulation with PMA-ionomycin in the presence of brefeldin A. Biotinylated human MR1 tetramers loaded with the active ligand 5-(2-oxopropylideneamino)6-D-ribitylaminouracil were used to confirm MAIT cell identification. MR1 tetramers were coupled to streptavidin-phycoerythrin (National Institutes of Health Tetramer Core Facility).

For surface staining, staining of a $200-\mu$ l blood sample was performed in PBS containing $1 \%$ BSA and $0.05 \%$ sodium azide. After surface staining, cells were fixated using BD FACS Lysing Solution (BD Biosciences, 349202) according to the manufacturer's instructions. Data acquisition was performed using a BD Biosciences LSR Fortessa. Flow cytometric analyses were performed with FlowJo v10.6.2 (Tree Star).

For intracellular labeling, thawed PBMCs were treated with DNase (0.05 $\mathrm{mg} \mathrm{ml}^{-1}$; D4263, Sigma-Aldrich) in RPMI (61870-010, Gibco) and incubated at $37^{\circ} \mathrm{C}$ and $5 \% \mathrm{CO}_{2}$ for $30 \mathrm{~min}$. Depending on the number of PBMCs obtained from each patient, intracytoplasmic staining of cytokines and $\mathrm{GzB}$ was analyzed with stimulation with PMA (P-8139, Sigma-Aldrich), ionomycin (I-0634, Sigma-Aldrich) and brefeldin A (B-7651, Sigma-Aldrich) and/or only brefeldin A. Stimulated cells were incubated for $6 \mathrm{~h}$ at $37^{\circ} \mathrm{C}$ in RPMI medium supplemented with $10 \%$ FCS, $1 \%$ HEPES, $1 \%$ penicillin-streptomycin and stimulated with PMA $\left(25 \mathrm{ng} \mathrm{ml}^{-1}\right)$ and ionomycin $\left(1 \mu \mathrm{g} \mathrm{ml}^{-1}\right)$ in the presence of brefeldin $\mathrm{A}\left(10 \mu \mathrm{g} \mathrm{ml}^{-1}\right)$. After surface staining, cells were fixed and permeabilized with a Cytofix/Cytoperm kit (554714, BD Biosciences) and then were washed using Perm/Wash buffer (BD Biosciences, 554723) and incubated at $4^{\circ} \mathrm{C}$ in the dark for $30 \mathrm{~min}$ with antibodies to cytokines and GzB (listed above).

For ETA and BAL samples, intracytoplasmic cytometry analysis was directly performed without PMA-ionomycin and brefeldin A stimulation.

In vitro culture. For virus culture, the Vero E6 kidney epithelial cell line was acquired from the American Type Culture Collection (CRL-1586; LGC standards). Vero E6 cells were checked for negative Mycoplasma presence, cultured in DMEM (Gibco) supplemented with $10 \%$ of heat-inactivated fetal bovine serum (Gibco; Thermo Fisher Scientific) and maintained at $37^{\circ} \mathrm{C}$ in a humidified atmosphere containing $5 \% \mathrm{CO}_{2}$. The viral strain of human SARS-CoV-2 was obtained from a nasopharyngeal positive PCR sample. SARS-CoV-2 PRIMO culture stock used in this study was produced in Vero E6 cells and titrated by lysis plaque assay ${ }^{49}$. SARS-CoV-2 stock titer was $2 \times 10^{7}$ p.f.u. $\mathrm{ml}^{-1}$. Supernatant was aliquoted for storage at $-80^{\circ} \mathrm{C}$.

For viral titration, SARS-CoV-2 was titrated by lysis plaque assay as previously described ${ }^{50}$. Vero E6 cells were plated onto 12 -well plate at a density of $5 \times 10^{4}$ cells per well in DMEM with $10 \%$ fetal bovine serum. At $24 \mathrm{~h}$ later, cells were infected by 10 to 10 serial viral dilutions. After virus adsorption for $1 \mathrm{~h}$ at $37^{\circ} \mathrm{C}$ with plate rocking every $15 \mathrm{~min}$, the viral inoculum was removed and Vero cells were washed with PBS-free medium. After, $500 \mu \mathrm{l}$ of an agarose medium mix was added. After $3 \mathrm{~d}$ of incubation at $37^{\circ} \mathrm{C}$ with $5 \% \mathrm{CO}_{2}$, supernatant was removed and cells were fixed with $1 \mathrm{ml}$ of $6 \%$ formalin solution for $30 \mathrm{~min}$. The formalin solution was removed, and cells were colored with a $10 \%$ crystal violet solution for $15 \mathrm{~min}$. All wells were then washed with distilled water and dried on bench-coat paper before analysis.

For macrophages that were infected by SARS-CoV-2 in vitro, CD14 ${ }^{+}$ monocytes were isolated using StraightFrom Whole Blood CD14 MicroBeads (130-090-879, Miltenyi Biotec). Then, $2 \times 10^{5}$ monocytes from each donor were added per culture well (96-well flat-bottom plate; 167008, Nunc). Monocytes were differentiated into macrophages by incubating CD14 ${ }^{+}$monocytes for $7 \mathrm{~d}$ in X-VIVO15 (BE02-060F, Lonza) with $50 \mathrm{ng} \mathrm{ml}^{-1}$ of GM-CSF (130-093-864, Miltenyi Biotec).

For infection, macrophages were washed twice in PBS and were treated with virus in nonsupplemented RPMI at a MOI of 0.3 and 3 as determined based on the viral titer and the number of cells plated, for 90 to $120 \mathrm{~min}$ at $37^{\circ} \mathrm{C}$. The macrophages were washed three times with PBS before adding the lysis buffer into each well. The intracellular viral load viral quantity was then determined by RTqPCR (see below).

PBMC preparation and coculture with macrophages. PBMCs were isolated from fresh blood samples using Lymphosep Lymphocyte Separation Media (LM-T1702/500, Biosera). B cells, monocytes and CD4 ${ }^{+}$cells were depleted from PBMCs of the same healthy donors using Dynabeads Untouched CD8 ${ }^{+}$ kit (11348D, Invitrogen) with an in-house antibody mix with the following biotinylated monoclonal anti-human antibodies: CD4 (OKT4), CD14 (63D3) and CD19 (HIB19; all from BioLegend). Next, $2 \times 10^{5}$ PBMCs or enriched MAIT cells per well were cocultured for either $24 \mathrm{~h}$ or $96 \mathrm{~h}$ with autologous differentiated macrophages previously infected or not with SARS-CoV-2. Blocking antibodies were used to IL-12p70 (MAB219, R\&D systems) at $5 \mu \mathrm{g} \mathrm{ml}^{-1}$, IL-18 (D044-3, $\mathrm{MBL})$ at $5 \mu \mathrm{g} \mathrm{ml}^{-1}$ and MR1 (361102, BioLegend) at $10 \mu \mathrm{g} \mathrm{ml}^{-1}$. Then, $1 \mu \mathrm{g} \mathrm{ml}^{-1}$ B18R (34-8185-81, Invitrogen) was used to block type I IFN ${ }^{51}$. After coculturing, macrophages were washed three times with PBS before adding the lysis buffer into each well. The intracellular viral quantity was then determined by RT-qPCR

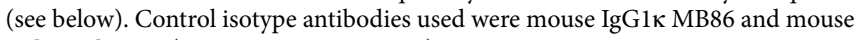
IgG2aк C76-47 (557353, BD Biosciences).

For the cytotoxicity assay, during the last $20 \mathrm{~h}$ of culture, dialyzed anti-CD107a monoclonal antibody $(0.5 \mu \mathrm{g}$ per well) was incubated with cells, and brefeldin A 
was added at $3 \mu \mathrm{g} \mathrm{ml}^{-1}$ in wells. Cells were then resuspended, transferred in tubes and washed in FACS buffer before surface staining or intracellular staining.

RNA extraction and quantitative PCR with reverse transcription. Total nucleic acids from cells and supernatant were extracted with the Total NA Isolation Kit-Large Volume assay on a MagNA Pure LC 2.0 analyzer (Roche). Nucleic acids were eluted in $50 \mu \mathrm{l}$ of elution buffer and immediately tested by qPCR of the albumin gene for cellular DNA ${ }^{52}$ and the RealStar SARS-CoV-2 RT-PCR Kit 1.0 (Altona Diagnostics) for SARS-CoV-2 detection. Viral RNA quantification was achieved using a standardized RNA transcript control acquired from the European Virus Archive program.

Blood MAIT cells and monocytes from patients with COVID-19 (IDU and ICU) were flow sorted and lysed in $\mathrm{RLT}^{+}$buffer with $1 \% \beta$-mercaptoethanol. mRNA was then extracted using an RNeasy Micro Kit (QIAGEN) according to the manufacturer's protocol.

Cytokine RNA quantification. Production of cDNA from total extracted nucleic acids was performed as described above using Superscript III reverse transcriptase (18080044; Invitrogen). Primers sequences used are described in Supplementary Table 2. Analysis by qPCR was performed with SYBR Green (4887352001; Roche) and was analyzed with a LightCycler 480 (Roche). Relative expression was calculated by the $2^{-\Delta \Delta \mathrm{Ct}}$ method and was normalized to expression of the housekeeping gene encoding $18 \mathrm{~S}$ and, when detailed, normalized on mock-infected macrophages.

Single-cell RNA-seq library preparation, sequencing and analysis. MAIT cells defined as $\mathrm{CD}^{+}{ }^{+} \mathrm{CD} 4^{-} \gamma \delta \mathrm{TCR}^{-} \mathrm{CD} 161^{\text {hi }} \mathrm{V} \alpha 7.2^{+}$tetramer $\mathrm{MR} 1^{+}$were flow sorted. Single-cell Gel Bead-In-EMulsions (GEMs) were generated using a Chromium Controller instrument (10x Genomics). Sequencing libraries were prepared using Chromium Single Cell 3' Reagent Kits (10x Genomics), according to the manufacturer's instructions. Briefly, GEM-RT was performed in a thermal cycler at $53^{\circ} \mathrm{C}$ for $45 \mathrm{~min}$ and $85^{\circ} \mathrm{C}$ for $5 \mathrm{~min}$. Post-GEM-RT Cleanup using DynaBeads MyOne Silane Beads was followed by cDNA amplification $\left(98^{\circ} \mathrm{C}\right.$ for $3 \mathrm{~min}$, cycled $12 \times 98^{\circ} \mathrm{C}$ for $15 \mathrm{~s}, 67^{\circ} \mathrm{C}$ for $20 \mathrm{~s}, 72^{\circ} \mathrm{C}$ for $1 \mathrm{~min}$ and $72^{\circ} \mathrm{C}$ for $1 \mathrm{~min}$ ). After a cleanup with SPRIselect Reagent Kit and fragment size estimation with High Sensitivity HS DNA kit run on a 2100 Bioanalyzer (Agilent), the libraries were constructed by performing the following steps: fragmentation, end repair, A-tailing, SPRIselect cleanup, adaptor ligation, SPRIselect cleanup, sample index PCR and SPRIselect size selection. The fragment size estimation of the resulting libraries was assessed with High Sensitivity HS DNA kit run on a 2100 Bioanalyzer (Agilent) and quantified using the Qubit dsDNA High Sensitivity HS assay (Thermo Fisher Scientific). Libraries were then sequenced by pair with a high output flowcell using an Illumina NextSeq 500 with the following modes: 26 bp (10x Index + UMI), 8 bp (i7 Index) and $57 \mathrm{bp}(\mathrm{read} 2)$

Sequenced data were demultiplexed with Aozan $^{53}$. The manufacturer's pipeline (Cell Ranger 4.0.0 (20-A)) was used to align, call cell barcodes, concatenate samples, eliminate debris and produce $t$-SNE dimension reduction plots according to recommended parameters. Results were visualized, statistically analyzed and colorized with Loupe Browser 4.2.0 (10x Genomics).

Statistics and bioinformatics analysis. All bioinformatics analyses were performed using RStudio (1.2.5) running on R software (4.0). We used the flow cytometry analysis R cytofWorkflow/CATALYST from Nowicka et al. ${ }^{54}$ to produce MDS plots of aggregated signal and normalized dimension reduction plots for UMAP and $t$-SNE with 500 MAIT cells per patient from Bichat hospital flow cytometry data. For correct script execution, this was combined with the packages ggcyto and CytoML. Our pipeline combined this workflow with the flowWorkspace R library for FlowJo workspace reading and cell population selection. Dplyr and tidyverse packages were used to sort and filter data. Multiparametric matrix correlation plots were produced with the adjusted rcorr function from Hmisc and RcmdrMisc packages to compute matrices of Spearman's correlations along with the pairwise $P$ values corrected for multiple inferences using Holm's method and visualized with the Corrplot and ggplot2 packages. Ggplot2 and ggpubr were used for production of R-based graphs. Correlation plots hierarchical clustering were produced with the hclust function included in the Corrplot library. Observations were filtered for missing values, and only complete observations were used. PCA was processed with FactoMineR library and graphically produced with Factoextra package. Heat maps were plotted using pheatmap library, with data centered to zero and scaled for each parameter. Clinical table values were computed with the atable R library.

Statistical analyses were performed with GraphPad Prism version 8.0.2 and $\mathrm{R}$ software version 4.0. All datasets were tested for normal distribution using the Shapiro-Wilk normality test. Since all normality tests returned negative, all data sets were compared using either non-parametric two-tailed Mann-Whitney tests or, if paired, using the Wilcoxon signed-rank test. Correlation calculation between two parameters was performed using Spearman's correlation test corrected for multiple inferences using Holm's method. Logistic regression and ROC curves were produced with XLSTATS 2020.4 (Addinsoft) and confirmed with a randomly split cohort on R with the RORC package. Prognostic validity of the model was evaluated by analysis of the ROC curve and was measured using the AUC. Differences were considered significant at $P<0.05\left({ }^{*} / \$, P<0.05 ;{ }^{* *} / \$ \$, P<0.01\right.$; $\left.\star * * / \$ \$ \$, P<0.001 ;{ }^{* * * *} / \$ \$ \$, P<0.0001\right)$

Reporting Summary. Further information on research design is available in the Nature Research Reporting Summary linked to this article.

\section{Data availability}

All datasets corresponding to Figs. 1-8, Corrplots data and extended data figures have been uploaded to https://doi.org/10.6084/m9.figshare.c.5243447.v1. All scRNA-seq data have been uploaded to the Gene Expression Omnibus with the accession code GSE164386.

\section{Code availability}

All R scripts are available at https://github.com/MatthieuRouland/ MAIT-COVID19/.

\section{References}

49. Hasnain, S. E. et al. Host-pathogen interactions during apoptosis. J. Biosci. 28, 349-358 (2003).

50. Gordon, D. E. et al. A SARS-CoV-2 protein interaction map reveals targets for drug repurposing. Nature 583, 459-468 (2020).

51. Symons, J. A., Alcamí, A. \& Smith, G. L. Vaccinia virus encodes a soluble type I interferon receptor of novel structure and broad species specificity. Cell 81, 551-560 (1995).

52. Désiré, N. et al. Quantification of human immunodeficiency virus type 1 proviral load by a TaqMan real-time PCR assay. J. Clin. Microbiol. 39, 1303-1310 (2001).

53. Perrin, S., Firmo, C., Lemoine, S., Le Crom, S. \& Jourdren, L. Aozan: an automated post-sequencing data-processing pipeline. Bioinformatics 33, 2212-2213 (2017).

54. Nowicka, M. et al. CyTOF workflow: differential discovery in high-throughput high-dimensional cytometry datasets. F1000Res $\mathbf{6}$, 748 (2017).

\section{Acknowledgements}

We thank all the patients and the physicians, nurses and technician staff who helped with the study. We thank the Department of Biological Hematology and the Department of Biochemistry of Bichat-Claude Bernard University Hospital for measuring white blood cells and CRP. We are grateful to M. Diedisheim for discussion about biostatistics and bioinformatics and the National Institutes of Health Tetramer Core Facility for human MR1 tetramers. The laboratories of A.L. and R.C.M. are supported by ANR11-IDEX-0005-02 Laboratory of Excellence INFLAMEX and Fondation pour la Recherche Médicale (EQU201903007779 to A.L. and EQU201903007816 to R.C.M.). R.C.M. received a Université de Paris COVID-19 grant. The laboratory of A.L. is also supported by ANR-17-CE14-0002-01, ANR-19-CE14-0020, Fondation Francophone pour la Recherche sur le Diabète and Juvenile Diabetes Research Foundation. A.L., C.B., A.V.-P. and J.-F.G. are supported by the Recherche Hospitalo-Universitaire (RHU) QUID-NASH program (ANR-17-RHUS-009). We thank D. Valla and A. Brzustowski, coordinator and manager of the RHU QUID-NASH. M.R. and L. Bertrand were supported by French Ministry of Research grants. A.T., Z.G. and L.C. were supported by RHU QUID-NASH. C.R. was supported by Fondation Francophone pour la Recherche sur le Diabète. P.S. was supported by the Juvenile Diabetes Research Foundation. We also thank Servier Medical Art for the free medical images, licensed under the Creative Commons Attribution 3.0 Unported License.

\section{Author contributions}

L. Bertrand and S. Lebourgeois contributed equally. H.F. collected numerous samples and clinical data and performed experiments. M.R. performed experiments and most of the bioinformatics analyses. L. Beaudoin performed experiments, analyzed data and managed experimental procedures. A.T., L. Bertrand and S. Lebourgeois performed experiments and analyzed data. C.R., P.S. and Z.G. performed flow cytometry experiments and analyses. L.C. helped with flow cytometry experiments. A.C.M. performed viral quantification. M.H.-N. performed CBA experiments. S. Luce collected samples. K.B. and M.A. performed MSD Quickplex experiments. B.S. and F.L. performed 10x Genomics experiments. Y.J., M.S.-T., T. B. and C.P. performed most of the ETA analyses. C.B., A.V.-P., J.-F.G., N.A., B.T., F.P., J.G., X.L., Y.Y. and J.-F.T. performed patient recruitment and analyzed clinical parameters. B.V. and D.D. isolated and characterized SARS-CoV-2. H.F., M.R., L. Beaudoin, A.T., L. Bertrand, S. Lebourgeois, R.C.M. and A.L. wrote the manuscript. H.F., L. Beaudoin, R.C.M. and A.L. conceived the study. A.L. supervised the study. All authors edited and approved the manuscript.

\section{Competing interests}

The authors declare no competing interests. 


\section{Additional information}

Extended data is available for this paper at https://doi.org/10.1038/s41590-021-00870-z. Supplementary information The online version contains supplementary material available at https://doi.org/10.1038/s41590-021-00870-z.

Correspondence and requests for materials should be addressed to A.L.
Peer review information Nature Immunology thanks Derya Unutmaz and the other anonymous, reviewer(s) for their contribution to the peer review of this work. Peer reviewer reports are available. Zoltan Fehervari was the primary editor on this article and managed its editorial process and peer review in collaboration with the rest of the editorial team.

Reprints and permissions information is available at www.nature.com/reprints. 


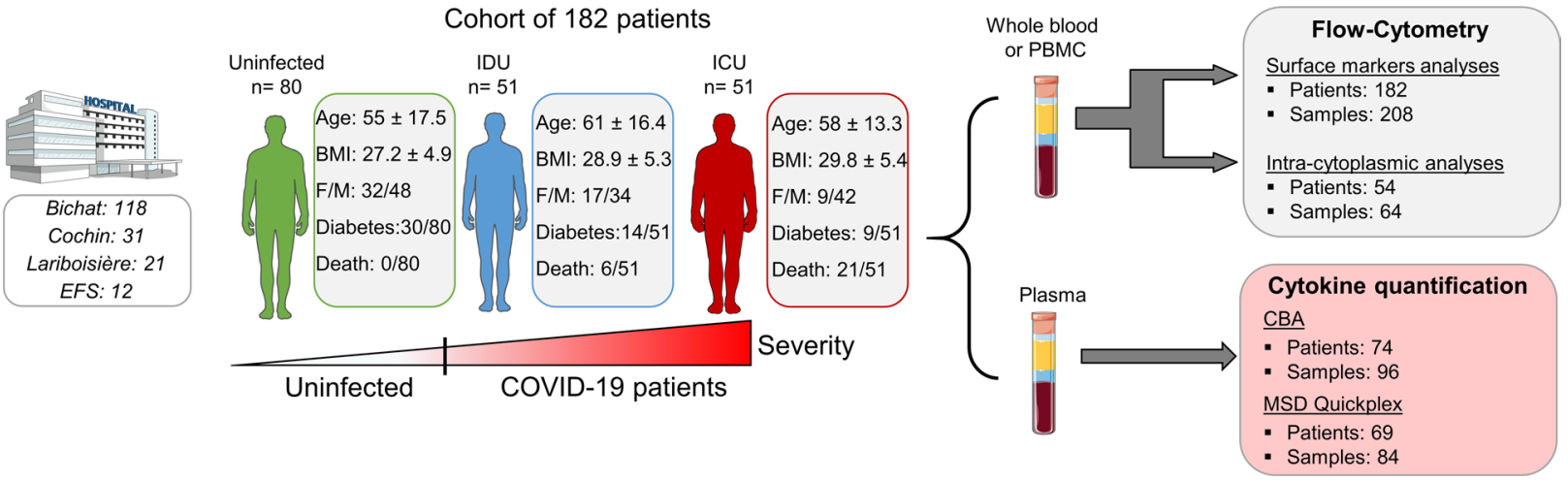

Extended Data Fig. 1 | Clinical study design of the first COVID-19 patient cohort. Related to Figs. 1-6. Graphical representation and experiment design flowchart for the first COVID-19 cohort including 182 patients from different hospitals and described as following: age, $B M I, \operatorname{sex}(F=F e m a l e, M=M a l e)$, diabetes, and fatality rates. Mean $( \pm S D$ ) values for each medical ward are represented. Illustration with images from Servier Medical Art, licensed under the Creative Commons Attribution 3.0 Unported License. 
a
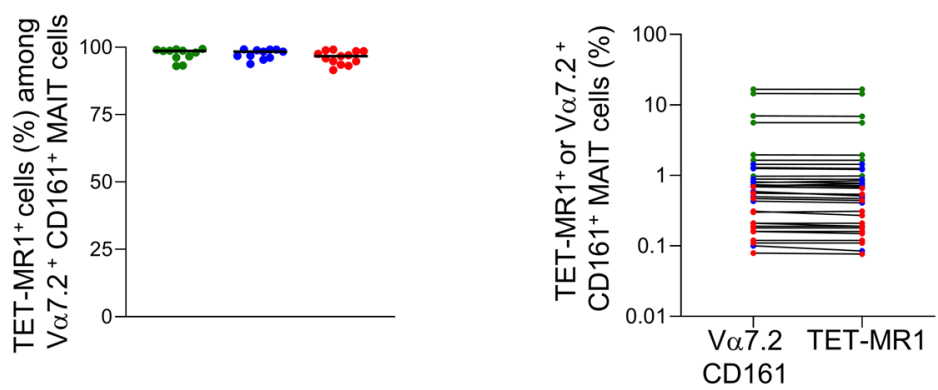

- Uninfected

Infectious Disease Unit (IDU)

Intensive Care Unit (ICU)

b
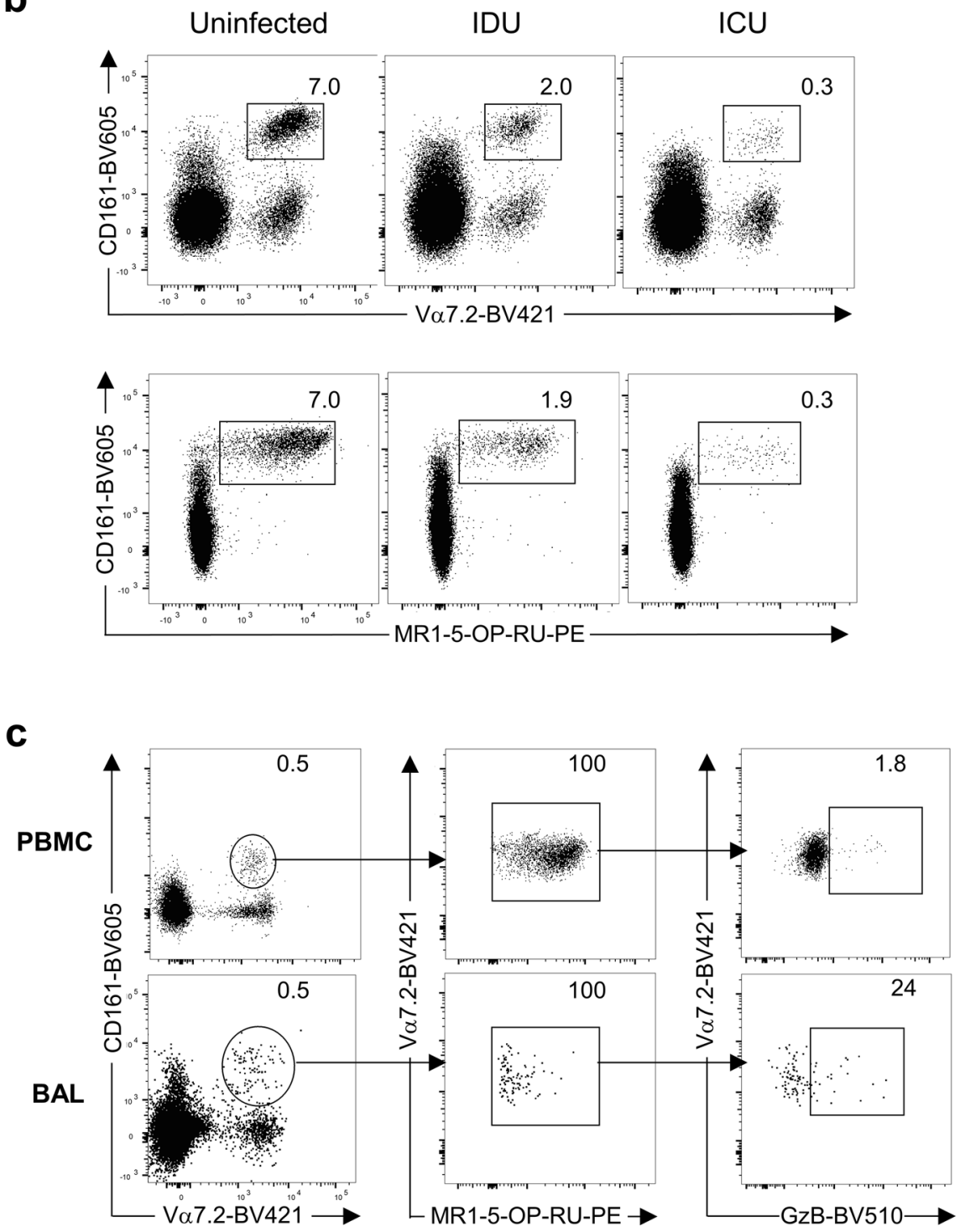

Extended Data Fig. 2 | MAIT cells co-staining with MR1-Tetramer and $\mathbf{V} \boldsymbol{\alpha} 7.2^{+}$CD161 $^{+}$. a, Flow cytometry analysis of 5-(2-oxopropylideneamino)6-D-ribitylaminouracil (5-OP-RU)-loaded MR1 tetramer binding among $V \alpha 7.2^{+}$CD161+ MAIT cells in uninfected patients $(n=11)$ and SARS-CoV-2 infected patients from Infectious Disease Unit (IDU) $(n=11)$ and Intensive Care Unit (ICU) $(n=13)$. b, Dot plot representative of MR1-Tetramer ${ }^{+}$CD161 ${ }^{+}$MAIT cells in one uninfected control and one COVID-19 patient from IDU and one from ICU. c, Dot plot representative of CD $161^{+} \mathrm{V}_{\alpha} 7.2^{+} \mathrm{Tetramer} M R 1^{+} \mathrm{GzB} \mathrm{B}^{+}$in PBMC and (Bronchoalveolar lavage) BAL from one ICU patients. 
a
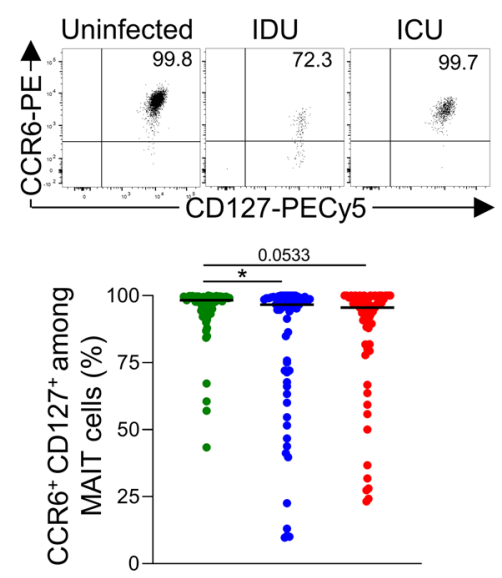

Uninfected

- Infectious Disease Unit (IDU)

- Intensive Care Unit (ICU)

- Deceased

b

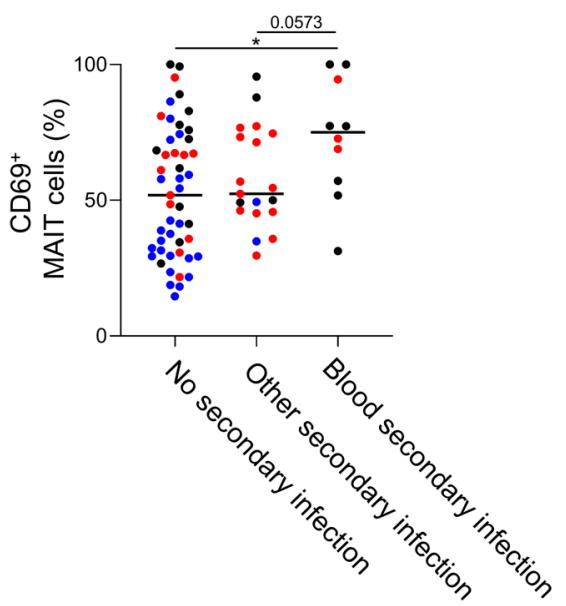

C

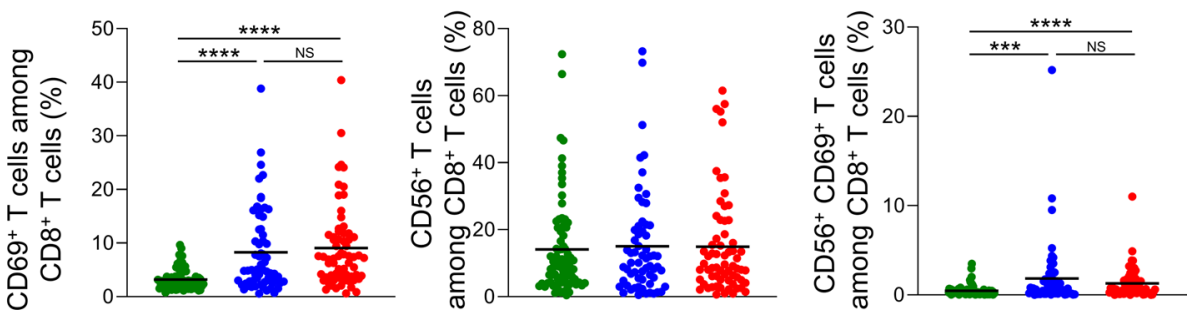

Extended Data Fig. 3 | MAIT and CD8 surface marker expression. a, Representative dot plot and frequency of CCR6 ${ }^{+}$CD127 $7^{+}$MAIT cells in uninfected controls $(n=80)$, IDU $(n=62)$, and ICU $(n=66)$ with SARS-CoV-2 infection. $\mathbf{b}$, Frequency of CD69+ MAIT cells in patients with COVID-19 who did not contract any secondary infection $(n=49)$, those who develop secondary infection outside blood circulation (for example Hospital acquired pneumonia, urine infections) $(n=19)$ and inside blood circulation (for example blood bacteriemia, septic shock) $(n=10)$. c, Flow cytometry analysis of CD69+, CD56+, and $\mathrm{CD}_{56}{ }^{+} \mathrm{CD} 69^{+} \mathrm{CD} 8^{+}$cells in uninfected controls $(n=80)$, IDU $(n=62)$, and ICU $(n=66)$ with SARS-CoV-2 infection. Each symbol represents a single biological sample. ${ }^{\star} \mathrm{P}<0.05,{ }^{\star \star} \mathrm{P}<0.01,{ }^{\star \star \star} \mathrm{P}<0.001$, and ${ }^{\star \star \star \star} \mathrm{P}<0.0001$ (two-sided Mann-Whitney nonparametric test). 
a

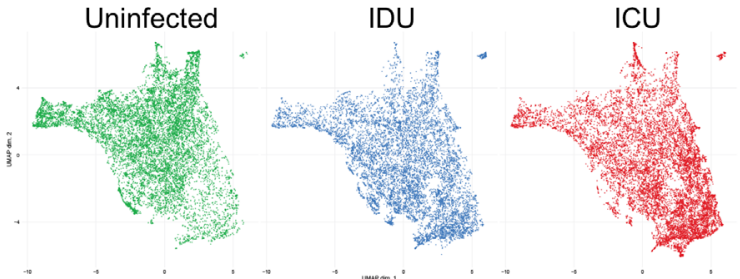

Uninfected

IDU

ICU

b

C
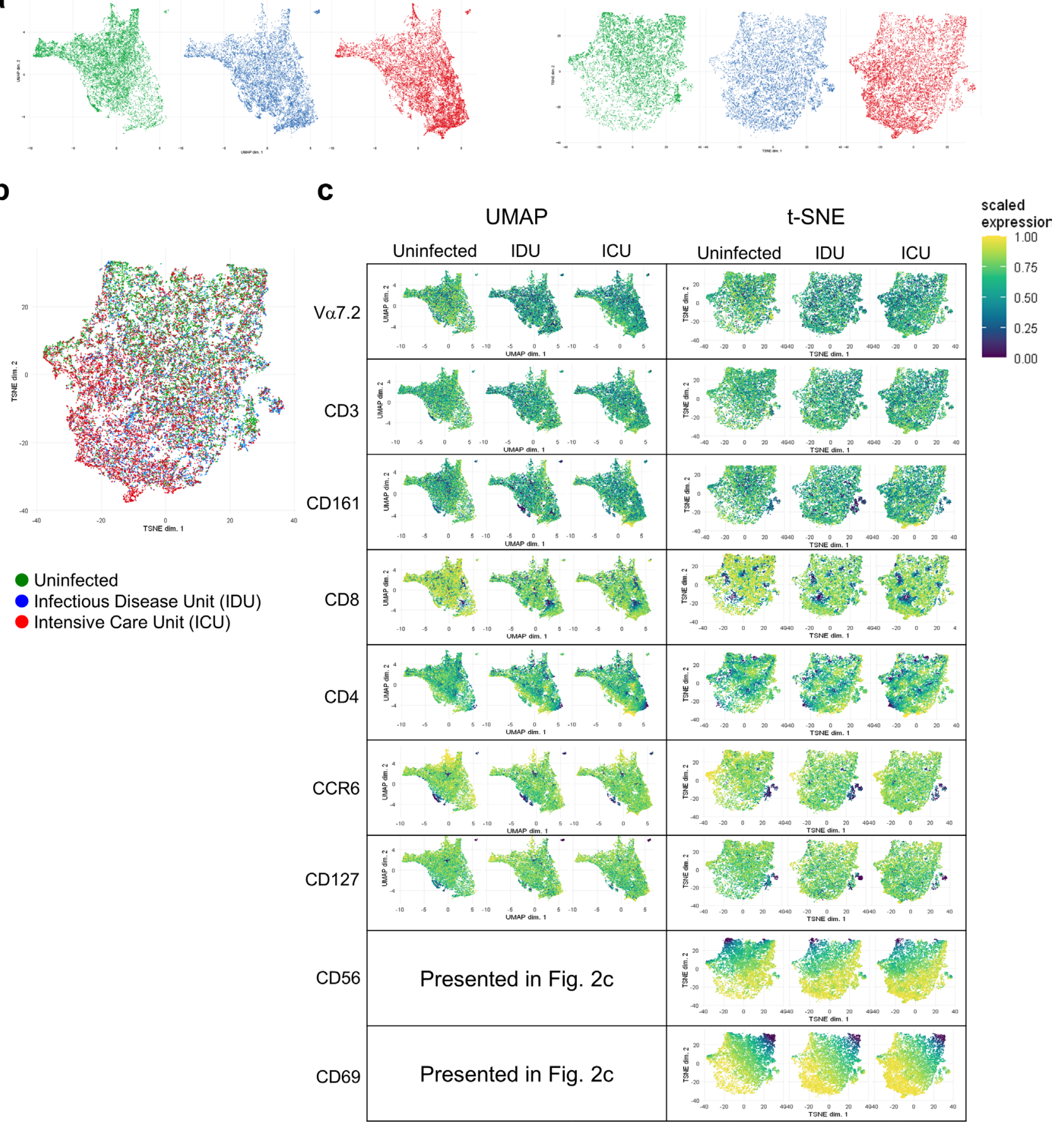

Uninfected

- Infectious Disease Unit (IDU)

Intensive Care Unit (ICU)

.

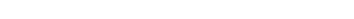

Extended Data Fig. 4 | Details of MAIT cell unsupervised analyses in COVID-19 patients. Dimensionality reduction plots including Uniform manifold approximation and projection (UMAP) and T-distributed Stochastic Neighbor Embedding (t-SNE) from uninfected, and infected patients presented in Fig. 2b,c. a, UMAP and t-SNE representation of MAIT cells from COVID-19 patients and non-infected controls separately. $\mathbf{b}$, t-SNE of MAIT cells from COVID-19 patients and uninfected controls. (c) UMAP and tSNE colored by the scaled expression of $\vee \alpha 7.2, C D 3, C D 161, C D 8, C D 4, C C R 6, C D 127, C D 56$, and CD69 specific markers in uninfected $(n=23), \operatorname{IDU}(n=50)$, and ICU patients $(n=66)$. 
a
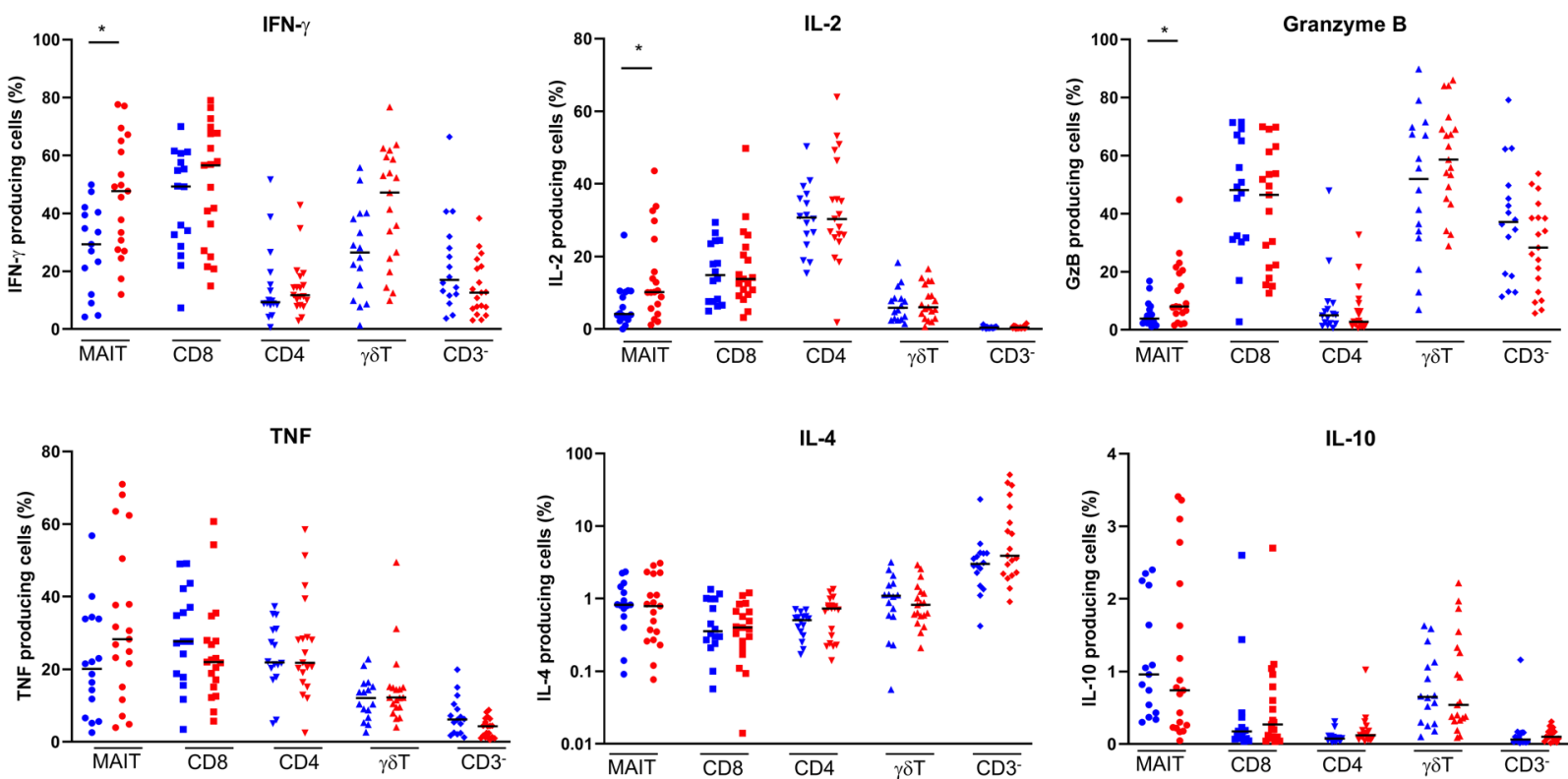

\section{b}

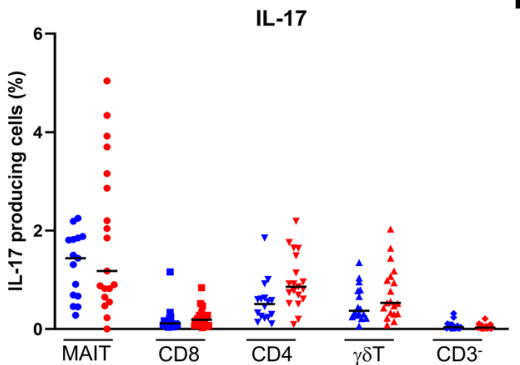

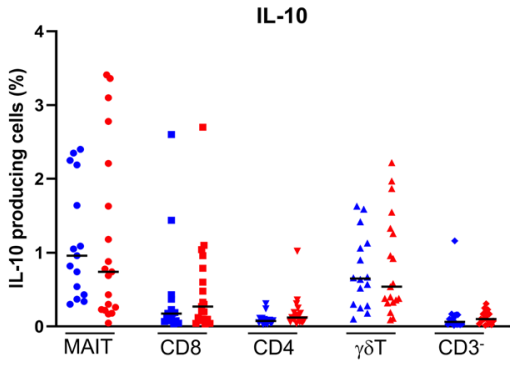

Uninfected

Infectious Disease Unit (IDU)

Intensive Care Unit (ICU)
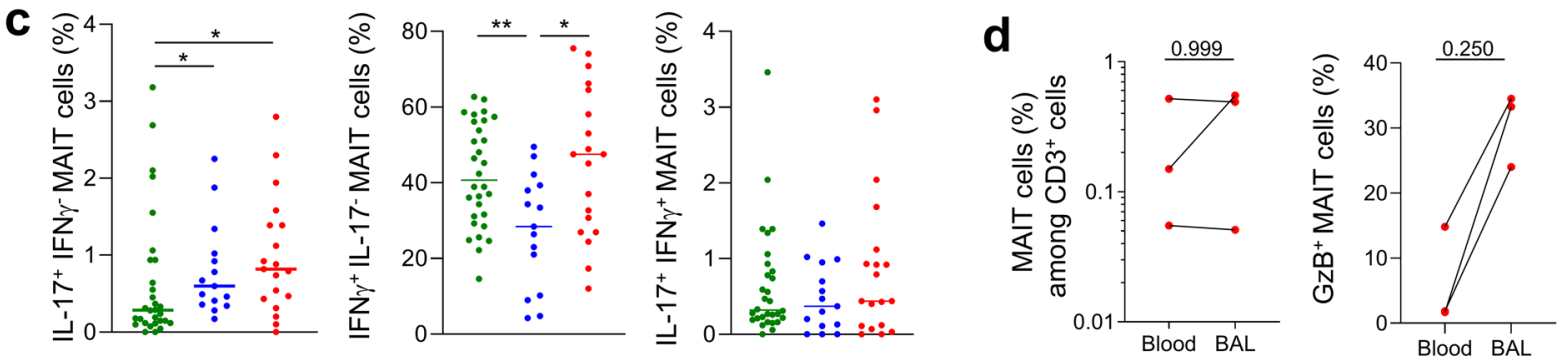

Extended Data Fig. 5 | MAIT cell function alterations in blood and bronchoalveolar lavage. a, Flow cytometry analysis of IFN $\gamma$, TNF, IL-2, IL-4, IL-10, IL-17, and granzyme B production among stimulated MAIT cells, conventional CD8 ${ }^{+}$and $C D 4^{+} T$ cells, $\gamma \delta$ T and $C D 3^{-}$cells from IDU ( $\left.n=15-16\right)$ or ICU COVID-19 patients $(n=19)$. b. Flow cytometry analysis of $G z B$ without stimulation from IDU $(n=11)$ or ICU COVID-19 patients $(n=14)$. c, Flow cytometry analysis of IL-17+, IFN $\gamma^{+}$, and double positive MAIT cells from Uninfected patients $(n=30)$, IDU $(n=11)$ or ICU COVID-19 patients $(n=14)$. $\mathbf{d}$, Flow cytometry analysis of MAIT cells and GzB+ MAIT cells frequencies in BAL fluids (Bronchoalveolar lavage) from 3 COVID-19 patients admitted in ICU and compared to their respective blood counterpart. Each symbol represents a single biological sample. ${ }^{\star} P<0.05$ and ${ }^{\star \star} P<0.01$ (two-sided Mann-Whitney nonparametric test (a-c) or Wilcoxon signed-rank test $(\mathbf{d})$ ). 
a

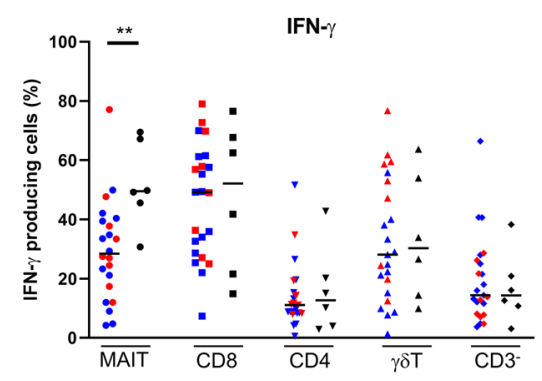

- Infectious Disease Unit (IDU)

Intensive Care Unit (ICU)
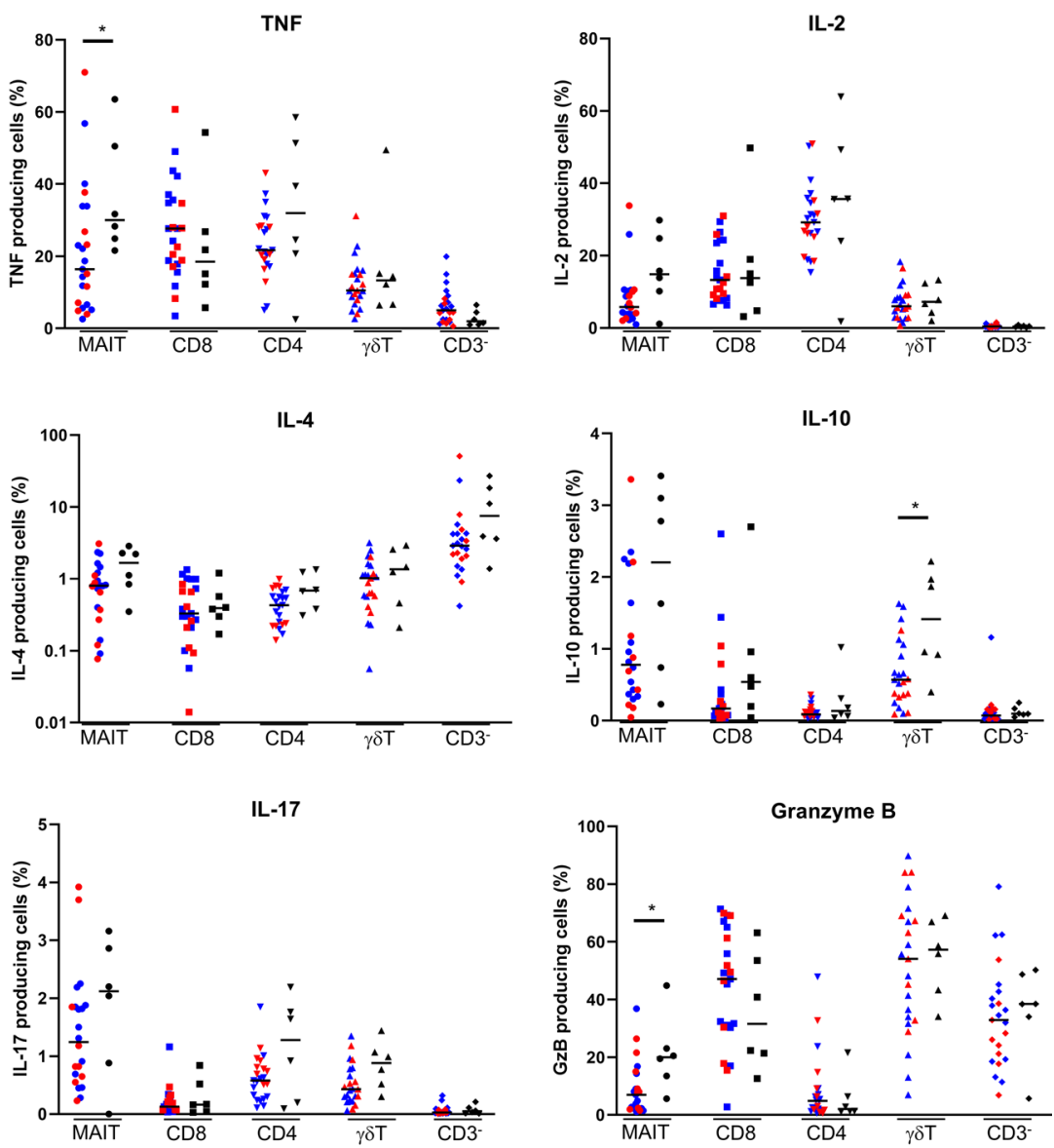

b

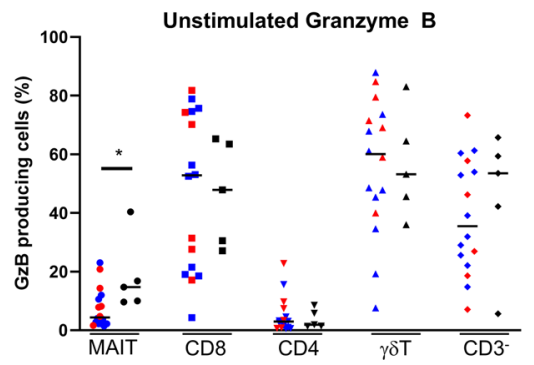

Extended Data Fig. 6 | MAIT cell functions are impacted in fatal SARS-CoV-2 infection. a, Flow cytometry analysis of IFN $\gamma$, TNF, IL-2, IL-4, IL-10, IL-17, and granzyme $B$ production among stimulated MAIT cells $(n=22)$, conventional CD $8^{+}$and CD4 ${ }^{+}$T cells, $\gamma \delta T$ and CD3- cells in non-fatal $(n=23)$ versus fatal COVID-19 patients $(n=6)$. b, Flow cytometry analysis of granzyme B among unstimulated MAIT cells, conventional CD $8^{+}$and $C D 4^{+} T$ cells, $\gamma \delta T$ and CD3- cells in non-fatal $(n=16)$ versus fatal COVID-19 patients $(n=5)$. Each symbol represents a single patient. ${ }^{\star} P<0.05$ and ${ }^{\star \star *} P<0.01$ (two-sided Mann-Whitney nonparametric test). 
a

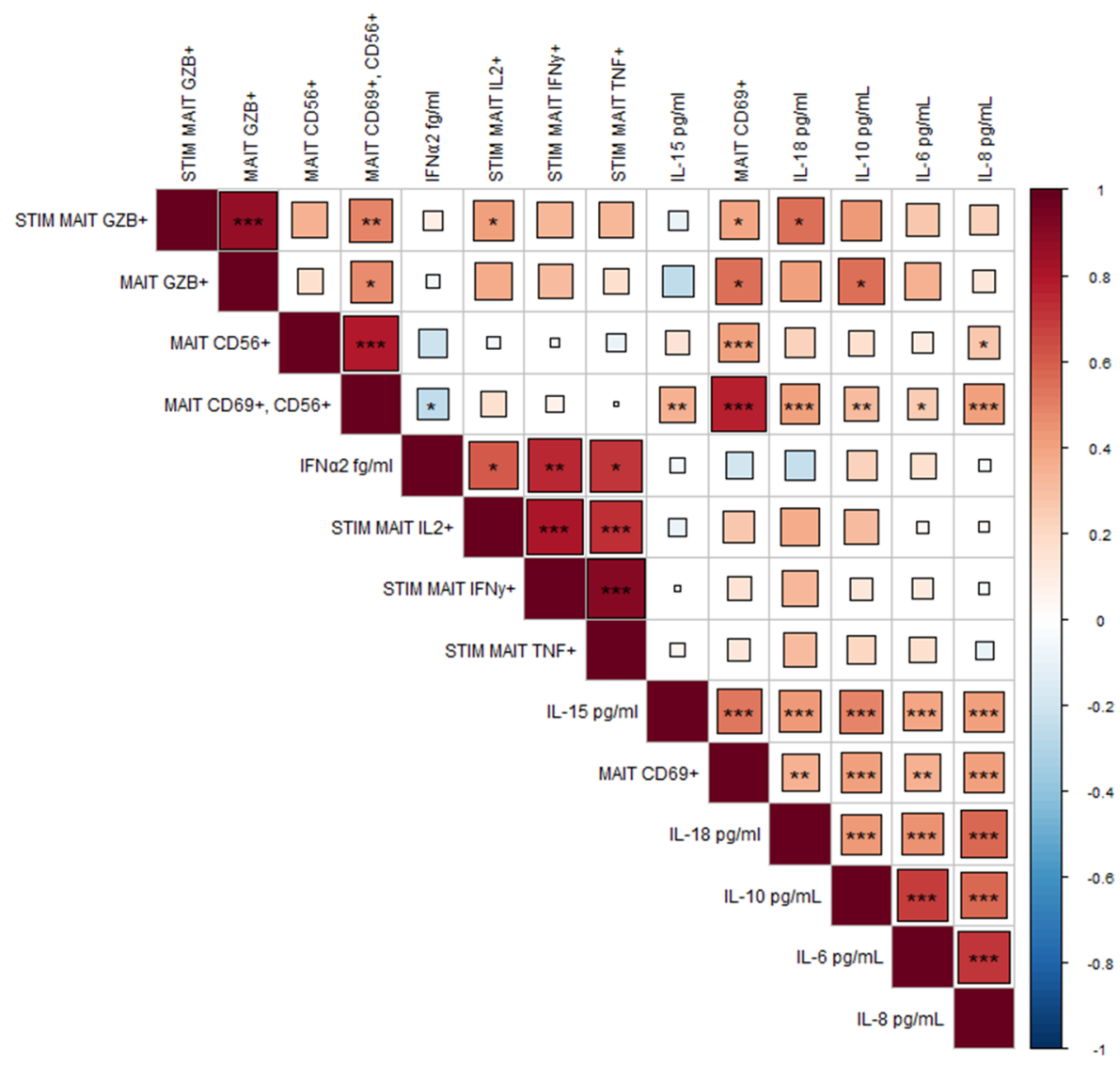

b
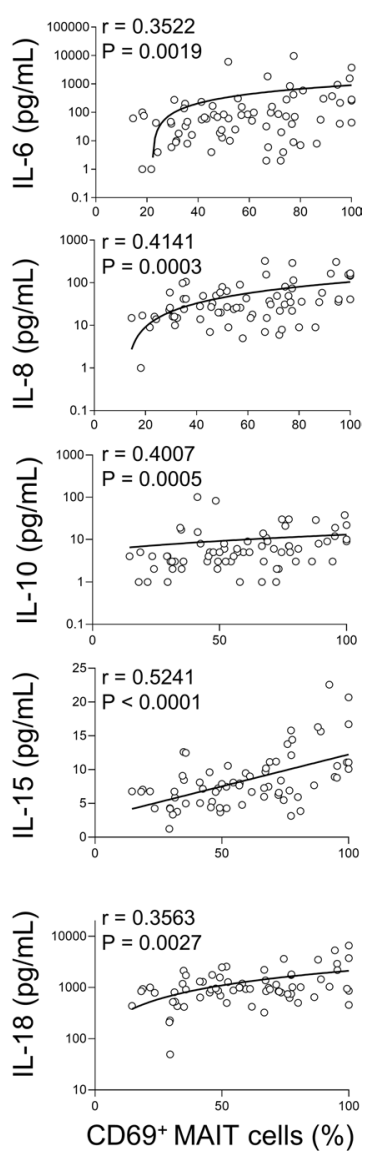

Extended Data Fig. 7 | Pro-inflammatory cytokine blood levels are correlated with MAIT cell activation and cytokine secretion. a, Multiparametric matrix correlation plot of plasmatic cytokine concentration, surface marker, and intracytoplasmic MAIT cell staining. Spearman's correlation coefficients are visualized by square size and color intensity. Variables are ordered by hierarchical clustering. $\mathbf{b}$, Correlation between various combinations (axes) of plasmatic cytokine concentrations (IL-6, IL-8, IL-10, IL-15, IL-18) and the frequency of CD69+ MAIT cells in patient's blood (presented as a \% value of total MAIT cells) $(n=69-93)$. Each symbol represents one single patient. ${ }^{\star} P<0.05,{ }^{\star \star} P<0.01$, and ${ }^{\star \star \star} P<0.001$ (Spearman nonparametric correlation test corrected for multiple inferences using Holm's method). 
a

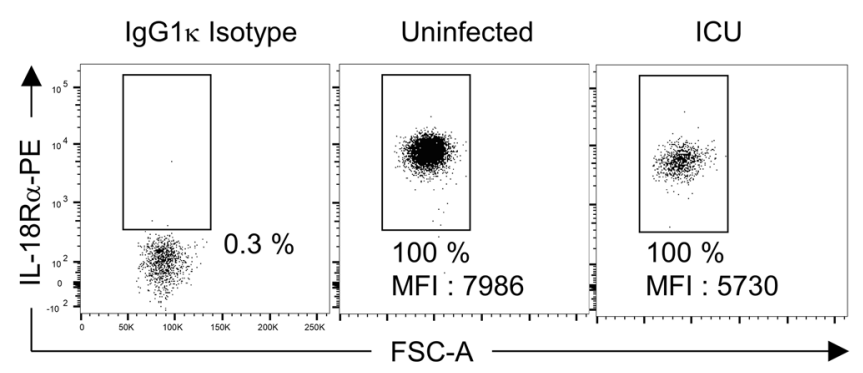

b

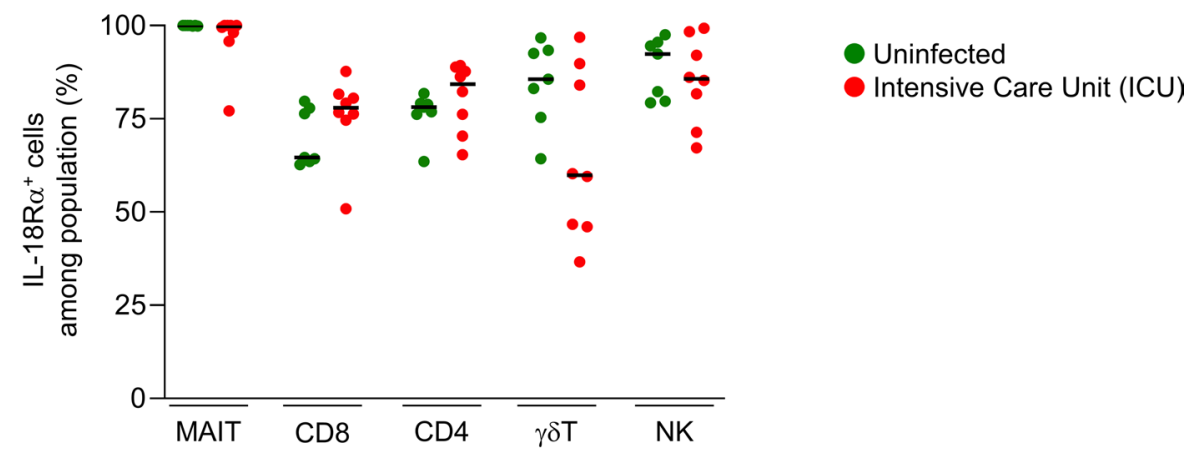

C

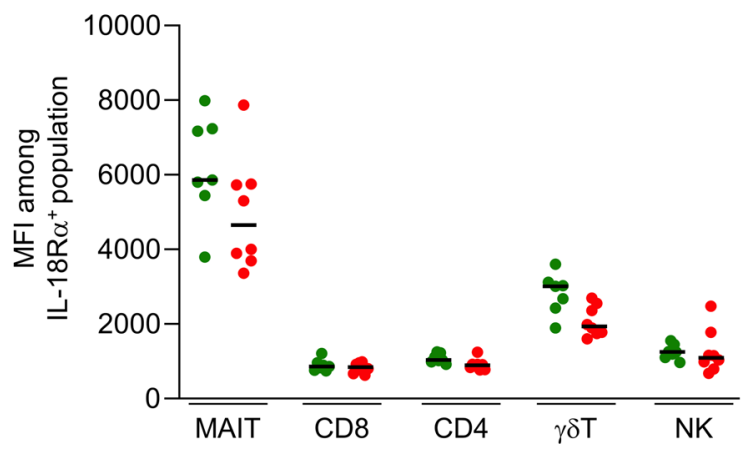

Extended Data Fig. 8 | IL-18 receptor is highly expressed on MAIT cells. a, Representative dot plot of IL-18R $\alpha$ MAIT cells in one uninfected control, and one COVID-19 patient from ICU. IgG1 $\kappa$ isotype is displayed as control staining. b,c, Frequencies (b) and Mean Fluorescence Intensity (MFI) (c) by flow cytometry, of IL-18 receptor among MAIT cells, conventional CD8 ${ }^{+}$and CD4+ $4^{+}$cells, $\gamma \delta T$, and NK cells in the blood of uninfected controls $(n=7)$ compared to COVID-19 patients hospitalized in Intensive Care Unit (ICU) $(n=8)$. FSC, Forward scatter. Small horizontal lines indicate the median, each symbol represents one single patient. 


\section{Cohort of 26 patients}
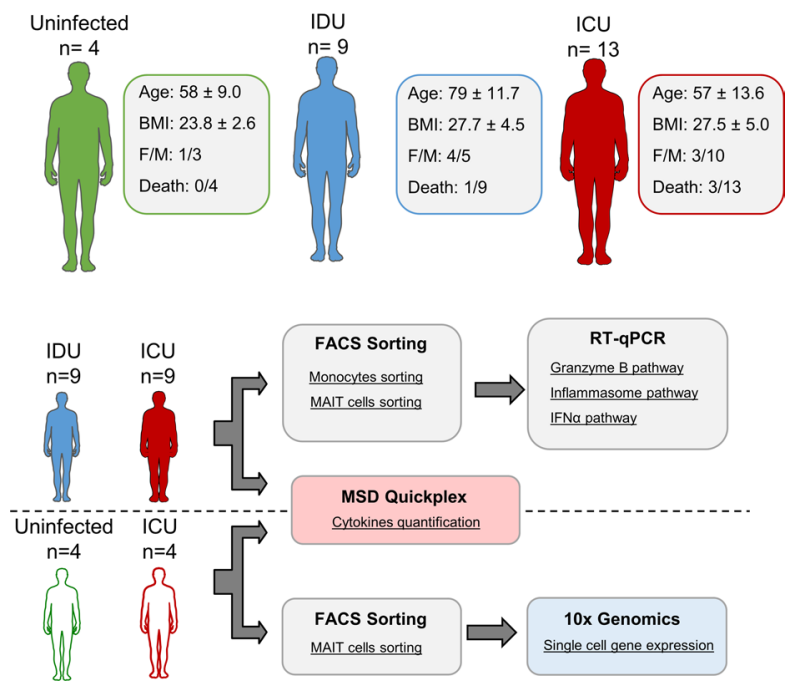

Extended Data Fig. 9 | Clinical study design of the second COVID-19 patient cohort. Related to Figs. 7 and 8. Graphical representation and experiment design flowchart for the second COVID-19 cohort including 26 patients from different hospitals and described as following: age, BMI, sex (F=Female, $M=$ Male) and fatality rates. Mean $( \pm S D)$ values for each medical ward are represented. Illustration with images from Servier Medical Art, licensed under the Creative Commons Attribution 3.0 Unported License. 
a
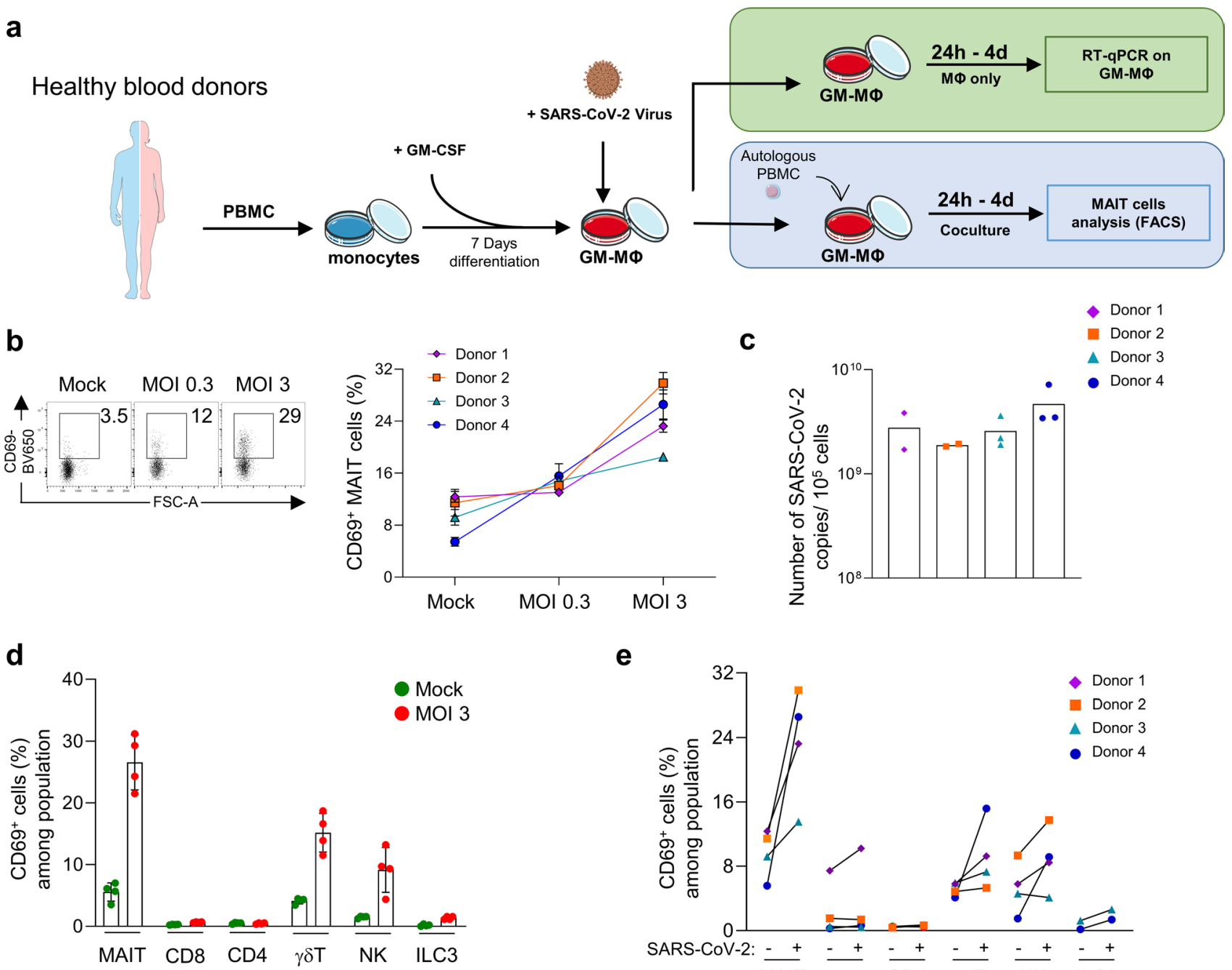

f
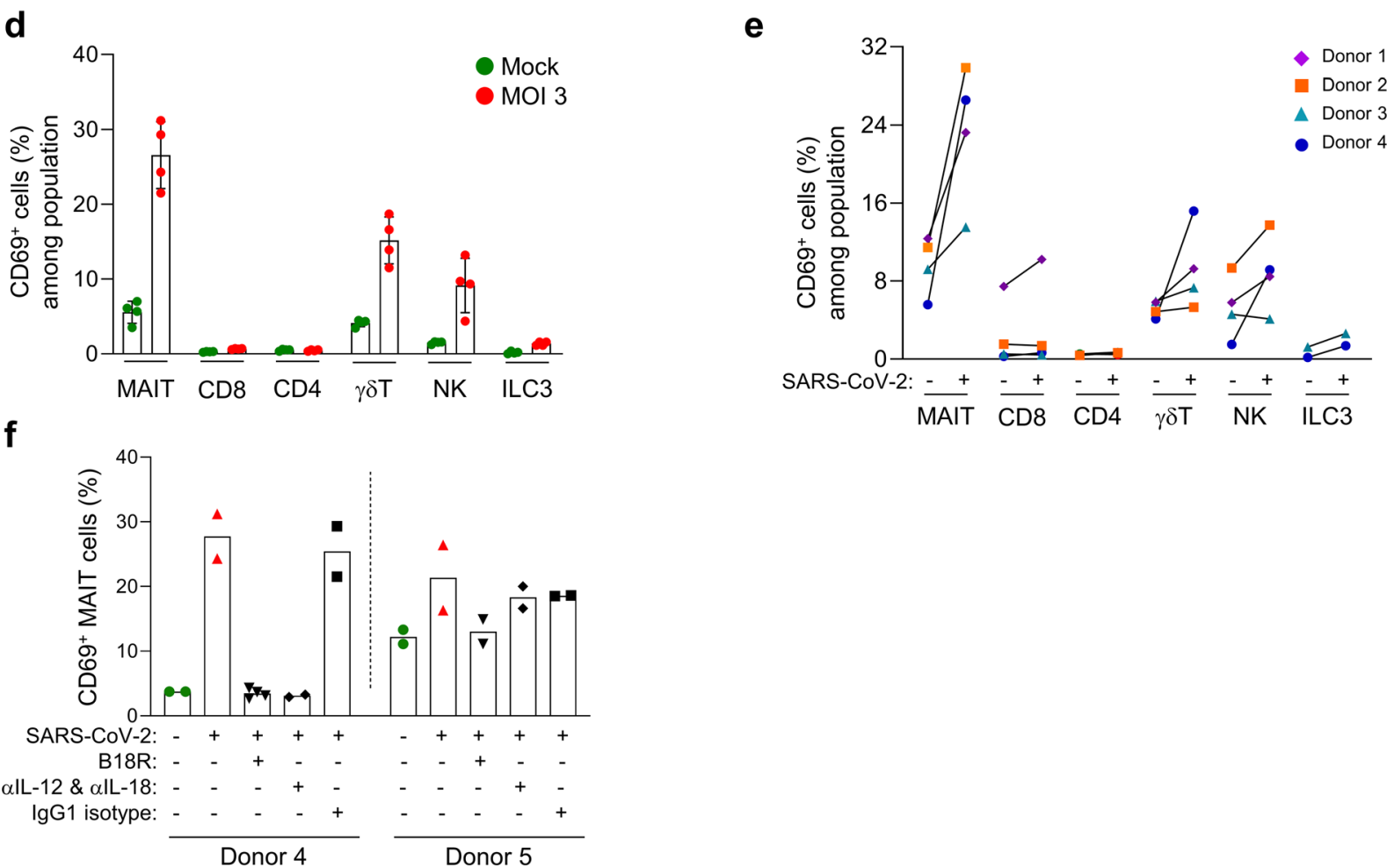

Extended Data Fig. 10 | Infected macrophages trigger MAIT cells activation in vitro. PBMCs of uninfected patients were challenged with infected macrophages by the SARS-CoV-2 virus. a, Graphical flowchart of the in-vitro co-culture experiments. b, Representative dot plot and frequency of CD69+ MAIT cells in healthy donors $(n=4)$ co-cultured with different SARS-CoV-2 Multiplicity of Infection (MOI) with mock, 0.3, and 3, with replicates. Each symbol represents the mean ( \pm SEM) value of replicates. c, Viral quantification of SARS-CoV-2 in co-culture from four donors at $96 \mathrm{~h}$, by RT-qPCR, with replicates (donor 1 and $2, n=2$, donor 3 and $4, n=3$ ). d,e, Frequency of CD69+ cells among MAIT cells, conventional CD8 ${ }^{+}$and $C D 4^{+} T$ cells, $\gamma \delta T, N K$, and ILC3 cells in donor $n^{\circ} 4(\mathbf{d})$ and all donors $(\mathbf{e})$ co-culture at $96 \mathrm{~h}$, with replicates $(n=2-4)$. Barplots represent mean $( \pm$ SD) values. $\mathbf{f}$, Co-culture of infected macrophages (MOI 3) with PBMC (donor 4) or purified T cells (donor 5) with or without anti-IL-12, anti-IL-18, or recombinant soluble IFN receptor protein (B18R), with replicates $(n=2-4)$. Each symbol represents a single co-culture well. Illustration with images from Servier Medical Art, licensed under the Creative Commons Attribution 3.0 Unported License. 


\section{Reporting Summary}

Nature Research wishes to improve the reproducibility of the work that we publish. This form provides structure for consistency and transparency in reporting. For further information on Nature Research policies, see our Editorial Policies and the Editorial Policy Checklist.

\section{Statistics}

For all statistical analyses, confirm that the following items are present in the figure legend, table legend, main text, or Methods section.

n/a Confirmed

$\bigotimes$ The exact sample size $(n)$ for each experimental group/condition, given as a discrete number and unit of measurement

\ A statement on whether measurements were taken from distinct samples or whether the same sample was measured repeatedly

The statistical test(s) used AND whether they are one- or two-sided

Only common tests should be described solely by name; describe more complex techniques in the Methods section.

\A description of all covariates tested

$\square$ \ A description of any assumptions or corrections, such as tests of normality and adjustment for multiple comparisons

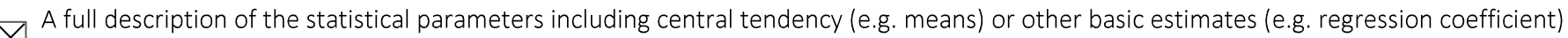

AND variation (e.g. standard deviation) or associated estimates of uncertainty (e.g. confidence intervals)

For null hypothesis testing, the test statistic (e.g. $F, t, r$ ) with confidence intervals, effect sizes, degrees of freedom and $P$ value noted

Give $P$ values as exact values whenever suitable.

Х $\square$ For Bayesian analysis, information on the choice of priors and Markov chain Monte Carlo settings

Х $\square$ For hierarchical and complex designs, identification of the appropriate level for tests and full reporting of outcomes

$\square \bigotimes$ Estimates of effect sizes (e.g. Cohen's $d$, Pearson's $r$ ), indicating how they were calculated

\section{Our web collection on statistics for biologists contains articles on many of the points above.}

\section{Software and code}

Policy information about availability of computer code

Data collection GPCR data were acquired using a LightCycler 480 (Roche). Flow cytometry Immunophenotyping data acquisition was performed using a BD LSRFortessa cytometer and cell sorting was performed using a BD Biosciences FACSFortessa and BD Biosciences FACSAria III Cell Sorter. Cytokine blood levels were assessed with MSD Quickplex using the U-plex Biomarqueur groupe 1 (human) Assay (K-15067L-1) and the Ultrasensitive assay S-PLEX Human IFN- $\alpha 2 a$ (K151P3S-1). For Cytometer Beads Array data acquisition was performed using a BD FACSLyric cytometer. Single-Cell RNA Sequencing data were generated with 10xGenomics Chromium Controller instrument and sequenced by Illumina Nextseq 500.

Data analysis

GraphPad PRISM software v8.0.2 (Statistics and graphics), RStudio v1.2.5, R software v4.0 (Statistics and graphics): cytofWorkflow v1.12, CATALYST v1.12.1, ggcyto v1.16.0, CytoML v2.0.0, FactoMineR v2.3, factoextra v1.0.7, RcmdrMisc v2.7-1, Hmisc v4.4-1, corrplot v0.84, ggplot2 v3.3.1, dplyr v1.0.0, ggpubr v0.4.0, flowWorkspace v4.0.2, atable v0.1.10, RORC v1.0-11, pheatmap v1.0.12 and tidyverse v1.3.0. XLSTATS 2020.4 (Addinsoft). FlowJo analysis software V10.6.2 (Tree Star). 10xGenomics Software: Cellranger V4.0.0 (20-A), Loupe V4.2.0. CBA: FCAP Array V3.0 (BD Biosciences) R scripts are available at https://github.com/MatthieuRouland/MAIT-COVID19. 
Policy information about availability of data

All manuscripts must include a data availability statement. This statement should provide the following information, where applicable:

- Accession codes, unique identifiers, or web links for publicly available datasets

- A list of figures that have associated raw data

- A description of any restrictions on data availability

All data sets corresponding to Fig. 1-8, Corrplots data and Extended Data Figures have been uploaded to https://doi.org/10.6084/m9.figshare.c.5243447.v1

All RNA single cell sequencing data have been uploaded to GEO. Access link will be given as soon as data have been verified by GEO.

\section{Field-specific reporting}

Please select the one below that is the best fit for your research. If you are not sure, read the appropriate sections before making your selection.

\Life sciences

Behavioural \& social sciences

Ecological, evolutionary \& environmental sciences

For a reference copy of the document with all sections, see nature.com/documents/nr-reporting-summary-flat.pdf

\section{Life sciences study design}

All studies must disclose on these points even when the disclosure is negative.

Sample size In the present study, no statistical methods were used to calculate sample size. First cohort includes a total of 182 patients. Cohort size was determined based on the number of patients admitted in infectious disease $(n=51)$ or intensive care units $(n=51)$ during the enrolling and sample collection period. 102 COVID-19 patients admitted in Bichat or Cochin hospitals between 03/23/2020 and 05/29/2020 were included. For comparisons, blood from 80 uninfected controls of the Quid-Nash project ( $n=30)$, Etablissement Français du Sang (EFS) $(n=12)$, and volunteer donors $(n=38)$ were collected.

Second cohort includes a total of 26 patients. Cohort size was determined based on the number of patients admitted in infectious disease ( $\mathrm{n}=$ 9) or intensive care unit $(n=13)$ during the second enrolling and sample collection period. 22 COVID-19 patients admitted in Bichat or Cochin hospitals between 06/29/2020 and 10/08/2020 were included. For comparisons, blood from 4 uninfected controls of volunteer donors was collected.

Data exclusions 220 were enrolled in this study, however 12 patients were excluded.

Pre-established enrolement criteria were stated as follows:

- COVID-19 PCR test not ambiguous.

- At least 10 MAIT cells in sample to ensure minimal representativity and accuracy of analysis.

Patients were excluded for one of the following reaseons:

- 7 excluded patients: COVID-19 PCR test ambiguous.

-5 excluded patients: amount of blood MAIT cells in sample $<10$ cells.

Replication $\quad$ For in vitro studies, at least two independent experiments were performed. For each co-culture, at least technical duplicates were always analyzed.

All attempts at independent experiments and replication were successful for in vitro studies.

Randomization No randomization was used in this study. Three differents groups were separated following infection status and medical ward of hospitalization. Patients included in the control group were selected from different sources and were taken randomly. Diabetes rate and BMI distribution of the control group matched those of the two groups of COVID-19 patients with a random selection of already collected patients from the QuidNash clinical project that enrolls a high proportion of diabetic and obese patients.

Blinding At time of data acquisition, scientists were not aware of the patients' immunological status. Aquisition team was different from analysis team.

Acquisition team: investigators were not blinded for patient wards (Healthy Control, Infectious Diseases Unit, Intensive Care Unit).

Data analysis team: investigators were not blinded for patient wards.

Patients' hospitalization wards are relevant for data analysis. Our study is designed on studying differences between COVID-19 patients issued from different medical wards reflecting severity of infection. Therefore it was not possible to make groups without this clinical information.

\section{Reporting for specific materials, systems and methods}

We require information from authors about some types of materials, experimental systems and methods used in many studies. Here, indicate whether each material, system or method listed is relevant to your study. If you are not sure if a list item applies to your research, read the appropriate section before selecting a response. 


\begin{tabular}{l|l}
\hline$n / a$ & Involved in the study \\
\hline & $\bigotimes$ Antibodies \\
\hline & $\bigotimes$ Eukaryotic cell lines \\
$\square$ & $\square$ Palaeontology and archaeology \\
$\square$ & $\square$ Animals and other organisms \\
$\square$ & $\square$ Clinical data \\
$\searrow$ & $\square$ Dual use research of concern
\end{tabular}

\section{Antibodies}

Antibodies used

\begin{abstract}
In the present study, the fluorochrome labeled anti-human antibodies for flow cytometry measurements in vitro and in vivo were: BV785-CD3 (1:20, clone OKT3, catalog n³17330; Biolegend); BV711-CD4 (1:33, clone OKT4, catalog n³17440; Biolegend); PECyanine5-CD4 (1:50, clone OKT4, catalog $n^{\circ} 317412$; Biolegend); APCCy7-CD8 (1:50, clone SK1, catalog $n^{\circ} 557834 ;$ BD Biosciences); BV421-TCR V $\alpha 7.2$ (1:50, clone 3C10, catalog n³51716; Biolegend); PECF594-TCR $\gamma \delta$ (1:10, clone B1, catalog $n^{\circ} 562511$; BD Biosciences); BV605-CD161 (1:20, clone HP-3G10, catalog n³39916; Biolegend); PE-CD196 (CCR6) (1:50, clone G034E3, catalog $n^{\circ}$ 353409; Biolegend); PECy7-CD25 (1:20, clone M-A251, catalog n 557741; BD Biosciences); PECy5-CD127 ( 1 :10, clone R34.34, catalog $n^{\circ}$ A64617; Beckman Coulter); BV510-CD56 (1:25, clone HCD56, catalog n 318340; BioLegend); BV650-CD69 (1:40, clone FN50, catalog $n^{\circ}$ 310934; BioLegend); FITC-IFNY (1:10, clone 4S B3, catalog ${ }^{\circ} 502506$; BioLegend); PE-IL2 (1 :20, clone MQ1-17H12, catalog $n^{\circ} 500307$; BioLegend); PECy7-IL4 (1:80, clone 8D4-8, catalog n560672 ; BDbiosciences); APC-IL10 (1:80, clone JES3-19F1, catalog $n^{\circ} 562036$; BDbiosciences); BV711-IL17a (1:40, clone BL168, catalog n512328 BioLegend); BV510-Granzyme B (1:20, clone GB11, catalog $n^{\circ} 563388$ BDbiosciences); BV650-TNF $\alpha$ (1:50, clone MAb11, catalog n502938; BioLegend); BV421-CD19 (1:400, clone HIB19, catalog $n^{\circ} 302234$; BioLegend); PE-CD218 $\alpha$ (IL-18R $\alpha$ ) (1:40, clone H44, catalog $n^{\circ} 564675$; BDbiosciences); PE-Mouse IgG1k, Isotype control (1:20, clone MOPC-21, catalog $n^{\circ} 555749$; BDbiosciences); PE-CD107a (1:20, clone eBioH4A3 catalog $n^{\circ} 12-1079-42$; eBioscience); FITC-CD16 (1:100, clone eBioCB16, catalog n¹1-0168-42; eBioscience ); PE-CD11b (1:100, clone CBRM1/5, catalog $n^{\circ}$ 301405; BioLegend); APC-CD19 (1:100, clone HIB19, catalog n³02211; BioLegend); BV42-CD19 (1:100, clone HIB19, catalog $n^{\circ}$ 302234; BioLegend); Pacific Blue-CD14 (1:100, clone M5E2, catalog n558121; BDbiosciences); Human MR1 5-OP-RU PE-labeled Tetramer (1:500; NIH Tetramer Core Facility);

Antibodies used for enriched MAIT cells experiment were: Biotin-CD4 (1:20, clone OKT4, catalog n³17406, BioLegend); Biotin-CD14 (1:20, clone 63D3 catalog n³67106, BioLegend); BiotinCD19 (1:20, clone HIB19 catalog n³02204, BioLegend).

For in vitro experiments, blocking antibodies were used:

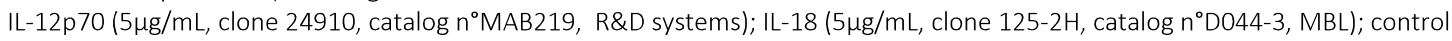
isotype IgG1 $\left(5 \mu \mathrm{g} / \mathrm{mL}\right.$, clone MB86, prepared by the A.L. laboratory); MR1 $\left(10 \mu \mathrm{g} / \mathrm{mL}\right.$, clone 26.5 , catalog $n^{\circ} 361102$, Biolegend), control isotype IgG2a (10 $\mathrm{\mu g} / \mathrm{mL}$, clone C76-47, catalog $\mathrm{n}^{\circ} 557353, \mathrm{BD}$ Biosciences).
\end{abstract}

Validation All antibodies were obtained from commercial vendors and validated by the producer. Futhermore, all antibodies were titrated and validated using appropriate controls.

\section{Eukaryotic cell lines}

Policy information about cell lines

Cell line source(s)

Vero E6 kidney epithelial cells line was acquired from the American Type Culture Collection (\#CRL-1586, ATCC, USA)

Authentication

CRL-1586, ATCC,USA, deposed by EM Earley, and routinely used in D. Descamps laboratory.

Mycoplasma contamination

Cell line was tested for mycoplasma and there is no contamination.

Commonly misidentified lines

(See ICLAC register)

None commonly misidentified lines were used.

\section{Human research participants}

Policy information about studies involving human research participants

Population characteristics

The first cohort is composed of 182 patients split into three groups: Uninfected ( $n=80)$, Infectious Disease Unit (IDU) ( $n=51)$, and Intensive Care Unit (ICU) ( $n=51$ ) COVID-19 patients.

Uninfected controls: Sex (Female: 40\% / Male: 60\%), Age (54.9 \pm 17.5$)$, BMI (27.2 \pm 4.9$)$.

IDU: Sex (Female: $33 \%$ / Male: $67 \%)$, Age $(61 \pm 16.4)$, BMI (28.9 \pm 5.3$)$.

ICU: Sex (Female: $18 \%$ / Male: $82 \%)$, Age (57.6 \pm 13.3$)$, BMI $(29.8 \pm 5.4)$.

Detailed clinical and immune data are included in supplementary table 1. 
The second cohort is composed of 26 patients splited in three groups: Uninfected $(n=4)$, Infectious Disease Unit (IDU) ( $n=9$ ), and Intensive Care Unit (ICU) ( $n=13$ ) COVID-19 patients.

Uninfected controls: Sex (Female: $25 \%$ / Male: 75\%), Age (58.0 \pm 9.0$),$ BMI $(23.8 \pm 2.6)$.

IDU: Sex (Female: 44\% / Male: 56\%), Age (79 \pm 11.7$)$, BMI (27.7 \pm 4.5$)$.

ICU: Sex (Female: $23 \%$ / Male: $77 \%)$, Age (57 \pm 13.6$)$, BMI (27.5 \pm 5.0$)$.

Clinical data presented below are mean values + SD

Recruitment

COVID-19 patients admitted to Bichat or Cochin hospitals with a positive SARS-CoV-2 PCR between 03/23/2020 and 05/29/2020 were included in the first cohort of this study. COVID-19 patients admitted to Bichat or Cochin hospitals with a positive SARS-CoV-2 PCR between 06/29/2020 and 10/08/2020 were included in the second cohort of this study. No self-selection bias were present in recruting healthy controls and COVID-19 patients.

Ethics oversight

The Ethics Committees approved clinical investigations. Informed consent was obtained from each enrolled patient. Patients from Bichat Hospital (Paris, France) were included in the French COVID cohort (NCT04262921). Ethics approval for this cohort was given on February 5th, 2020 by the French Ethics Committee CPP-Ile de France- VI (ID CRB: 2020-A00256-33). This cohort is sponsored by Inserm and supported by the REACTing consortium and by the French Ministry of Health (PHRC $n^{\circ}$ 20-0424). Samples from these patients were derived from samples collected in routine care. Patients from Cochin Hospital (Paris, France) were recruited in the setting of the local RADIPEM biological samples collection derived from samples collected in routine care. Biological collection and informed consent were approved by the Direction de la Recherche Clinique et Innovation (DRCI) and the French Ministry of Research ( $\left.N^{\circ} 2019-3677\right)$. Investigations with control patients from QUIDNASH were approved by Comité de Protection des Personnes de Sud Méditerranée (V) \#18.021, $N^{\circ}$ of QUID project registration: 2018-A00311-54. Lung fluid samples from Bichat hospital, samples were obtained under authorization from the ethics committee CPP- Ile de France XI (IDRCB: 2020-001457643, 20037-25957, APHP 200388-COVIDICUS). Other lung fluid samples were obtained from the Regional University Tours hospital (ClinicalTrial.gov identifier: NCT03379207) and under authorization from the ethics committee CPP-lle-de-France-VIII (IDRCB: 2017-A01841-52).

Note that full information on the approval of the study protocol must also be provided in the manuscript.

\section{Flow Cytometry}

Plots

Confirm that:

\The axis labels state the marker and fluorochrome used (e.g. CD4-FITC).

\The axis scales are clearly visible. Include numbers along axes only for bottom left plot of group (a 'group' is an analysis of identical markers).

\All plots are contour plots with outliers or pseudocolor plots.

\A numerical value for number of cells or percentage (with statistics) is provided.

\section{Methodology}

Sample preparation

Instrument

Software

Cell population abundance

Gating strategy
Whole blood samples were collected in EDTA or heparin-coated tubes (Vacutainer, BD Biosciences) from healthy, noninfected, donors; and COVID-19 patients admitted to Cochin, Bichat, or Lariboisière Hospitals in Paris, France. PBMC were isolated from heparinized blood samples by Ficoll-Plaque (Leucosep). Staining of $200 \mu \mathrm{L}$ of blood sample was performed in PBS containing $1 \%$ BSA and $0.05 \%$ sodium azide. After surface staining, cells were fixated using BD FACS Lysing Solution (BD Biosciences, 349202) according to the manufacturer's instructions.

BD LSRFortessa X-20, BD FACSARIA III Cell Sorter

Data was acquired with the Diva software (BD Biosciences) and analyzed with FlowJo V10.6.2 (TreeStar).

Cells populations were reported in various formats including a proportion of a specific population (\% of CD3+, \% of CD3-, etc.) or by parent gate (\% of MAIT cells, $\%$ CD8, etc.).

Gating strategies are described in the manuscript in Supplementary Information 1 and Supplementary Information 3.

Supplementary Information 1: Flow cytometry gating strategy for immune cell identification and functions. Surface gating strategies used in figures 1, 2, 3, 4, 7, 8. Intracytoplasmic gating strategies used in figures 2, 7.

Supplementary Information 3: Flow cytometry gating strategy for monocyte subset identification. Surface gating strategies used in figure 7.

$\bigotimes$ Tick this box to confirm that a figure exemplifying the gating strategy is provided in the Supplementary Information. 